\title{
Vector-Valued Jack Polynomials from Scratch
}

\author{
Charles F. DUNKL ${ }^{\dagger}$ and Jean-Gabriel LUQUE $\ddagger$ \\ $\dagger$ Dept. of Mathematics, University of Virginia, Charlottesville VA 22904-4137, USA \\ E-mail: cfd5z@virginia.edu \\ URL: http://people.virginia.edu/ cfd5z/ \\ ¥Université de Rouen, LITIS Saint-Etienne du Rouvray, France \\ E-mail: jean-gabriel.luque@univ-rouen.fr \\ URL: http://www-igm.univ-mlv.fr/ luque/
}

Received September 21, 2010, in final form March 11, 2011; Published online March 16, 2011 doi:10.3842/SIGMA.2011.026

\begin{abstract}
Vector-valued Jack polynomials associated to the symmetric group $\mathfrak{S}_{N}$ are polynomials with multiplicities in an irreducible module of $\mathfrak{S}_{N}$ and which are simultaneous eigenfunctions of the Cherednik-Dunkl operators with some additional properties concerning the leading monomial. These polynomials were introduced by Griffeth in the general setting of the complex reflections groups $G(r, p, N)$ and studied by one of the authors (C. Dunkl) in the specialization $r=p=1$ (i.e. for the symmetric group). By adapting a construction due to Lascoux, we describe an algorithm allowing us to compute explicitly the Jack polynomials following a Yang-Baxter graph. We recover some properties already studied by C. Dunkl and restate them in terms of graphs together with additional new results. In particular, we investigate normalization, symmetrization and antisymmetrization, polynomials with minimal degree, restriction etc. We give also a shifted version of the construction and we discuss vanishing properties of the associated polynomials.
\end{abstract}

Key words: Jack polynomials; Yang-Baxter graph; Hecke algebra

2010 Mathematics Subject Classification: 05E05; 16T25; 05C25; 33C80

\section{Introduction}

The Yang-Baxter graphs are used to study Jack polynomials. In particular, these objects have been investigated in this context by Lascoux [14] (see also [15, 16] for general properties about Jack and Macdonald polynomials). Vector-valued Jack polynomials are associated with irreducible representations of the symmetric group $\mathfrak{S}_{N}$, that is, to partitions of $N$. A YangBaxter graph is a directed graph with no loops and a unique root, whose edges are labeled by generators of a certain subsemigroup of the extended affine symmetric group. In this paper the vertices are labeled by a pair consisting of a weight in $\mathbb{N}^{N}$ and the content vector of a standard tableau. The weights are the labels of monomials which are the leading terms of polynomials, and the tableaux all have the same shape. There is a vector-valued Jack polynomial associated with each vertex. These polynomials are special cases of the polynomials introduced by Griffeth $[7,8]$ for the family of complex reflection groups denoted by $G(r, p, N)$ (where $p \mid r$ ). This is the group of unitary $N \times N$ matrices such that their nonzero entries are $r^{\text {th }}$ roots of unity, the product of the nonzero entries is a $(r / p)^{\text {th }}$ root of unity, and there is exactly one nonzero entry in each row and each column. The symmetric group is the special case $G(1,1, N)$. The vector space in which the Jack polynomials take their values is equipped with the nonnormalized basis described by Young, namely, the simultaneous eigenvectors of the Jucys-Murphy elements.

The labels on edges denote transformations to be applied to the objects at a vertex. Vectorvalued Jack polynomials are uniquely determined by their spectral vector, the vector of eigenvalues under the (pairwise commuting) Cherednik-Dunkl operators. This serves to demonstrate 
the claim that different paths from one vertex to another produce the same result, a situation which is linked to the braid or Yang-Baxter relations. These refer to the transformations.

Following Lascoux [14] we define the monoid $\widehat{\mathfrak{S}}_{N}$, a subsemigroup of the affine symmetric group, with generators $\left\{s_{1}, s_{2}, \ldots, s_{N-1}, \Psi\right\}$ and relations:

$$
\begin{aligned}
& s_{i} s_{j}=s_{j} s_{i}, \quad|i-j|>1, \\
& s_{i} s_{i+1} s_{i}=s_{i+1} s_{i} s_{i+1}, \quad 1 \leq i<N-1, \\
& s_{1} \Psi^{2}=\Psi^{2} s_{N-1}, \\
& s_{i} \Psi=\Psi s_{i-1}, \quad 2 \leq i \leq N-1 .
\end{aligned}
$$

The relations $s_{i}^{2}=1$ do not appear in this list because the graph has no loops.

The main objects of our study are polynomials in $x=\left(x_{1}, \ldots, x_{N}\right) \in \mathbb{R}^{N}$ with coefficients in $\mathbb{Q}(\alpha)$, where $\alpha$ is a transcendental (indeterminate), and with values in the $\mathfrak{S}_{N}$-module corresponding to a partition $\lambda$ of $N$. The Yang-Baxter graph $G_{\lambda}$ is a pictorial representation of the algorithms which produce the Jack polynomials starting with constants. The generators of $\widehat{\mathfrak{S}}_{N}$ correspond to transformations taking a Jack polynomial to an adjacent one. At each vertex there is such a polynomial, and a 4-tuple which identifies it. The 4-tuple consists of a standard tableau denoting a basis element of the $\mathfrak{S}_{N}$-module, a weight (multi-index) describing the leading monomial of the polynomial, a spectral vector, and a permutation, essentially the rank function of the weight. The spectral vector and permutation are determined by the first two elements. For technical reasons the standard tableaux are actually reversed, that is, the entries decrease in each row and each column. This convention avoids the use of a reversing permutation, in contrast to Griffeth's paper [8] where the standard tableaux have the usual ordering.

The symmetric and antisymmetric Jack polynomials are constructed in terms of certain subgraphs of $G_{\lambda}$. Furthermore the graph technique leads to the definition and construction of shifted inhomogeneous vector-valued Jack polynomials.

Here is an outline of the contents of each section.

Section 2 contains the basic definitions and construction of the graph $G_{\lambda}$. The presentation is in terms of the 4-tuples mentioned above. It is important to note that not every possible label need appear on edges pointing away from a given vertex: if the e weight at the vertex is $v \in \mathbb{N}^{N}$ then the transposition $(i, i+1)$ (labeled by $s_{i}$ ) can be applied only when $v[i] \leq v[i+1]$, that is, when the resulting weight is greater than or equal to $v$ in the dominance order. The action of the affine element $\Psi$ is given by $v \Psi=(v[2], v[3], \ldots, v[N], v[1]+1)$.

The Murphy basis for the irreducible representation of $\mathfrak{S}_{N}$ along with the definition of the action of the simple reflections $(i, i+1)$ on the basis is presented in Section 3 Also the vectorvalued polynomials, their partial ordering, and the Cherednik-Dunkl operators are introduced here.

Section 4 is the detailed development of Jack polynomials. Each edge of the graph $G_{\lambda}$ determines a transformation that takes the Jack polynomial associated with the beginning vertex to the one at the ending vertex of the edge. There is a canonical pairing defined for the vectorvalued polynomials; the pairing is nonsingular for generic $\alpha$ and the Cherednik-Dunkl operators are self-adjoint. The Jack polynomials are pairwise orthogonal for this pairing and the squared norm of each polynomial can be found by use of the graph.

In Section 5 we investigate the symmetric and antisymmetric vector-valued Jack polynomials in relation with connectivity of the Yang-Baxter graph whose affine edges have been removed. Also, in this section one finds the method of producing coefficients so that the corresponding sum of Jack polynomials is symmetric or antisymmetric. The idea is explained in terms of certain subgraphs of $G_{\lambda}$. 
Vertices of $G_{\lambda}$ satisfying certain conditions may be mapped to vertices of a graph related to $\mathfrak{S}_{M}, M<N$, by a restriction map. This topic is the subject of Section 6 . This section also describes the restriction map on the Jack polynomials.

In Section 7 the shifted vector-valued Jack polynomials are presented. These are inhomogeneous and the parts of highest degree coincide with the homogeneous Jack polynomials of the previous section. The construction again uses the Yang-Baxter graph $G_{\lambda}$; it is only necessary to change the operations associated with the edges.

Throughout the paper there are numerous figures to concretely illustrate the structure of the graphs.

\section{$2 \quad$ Yang-Baxter type graph associated to a partition}

\subsection{Sorting a vector}

Consider a vector $v \in \mathbb{N}^{N}$, we want to compute the unique decreasing partition $v^{+}$, which is in the orbit of $v$ for the action of the symmetric group $\mathfrak{S}_{N}$ acting on the right on the position, using the minimal number of elementary transpositions $s_{i}=(i i+1)$.

If $v$ is a vector we will denote by $v[i]$ its $i$ th component. Each $\sigma \in \mathfrak{S}_{N}$ will be associated to the vector of its images $[\sigma(1), \ldots, \sigma(N)]$. Let $\sigma$ be a permutation, we will denote $\ell(\sigma)=\min \{k$ : $\left.\sigma=s_{i_{1}} \cdots s_{i_{k}}\right\}$ the length of the permutation. By a straightforward induction one finds:

Lemma 2.1. Let $v \in \mathbb{N}^{N}$ be a vector, there exists a unique permutation $\sigma_{v}$ such that $v=v^{+} \sigma_{v}$ with $\ell\left(\sigma_{v}\right)$ minimal.

The permutation $\sigma_{v}$ is obtained by a standardization process: we label with integer from 1 to $N$ the positions in $v$ from the largest entries to the smallest one and from left to right.

Example 2.2. Let $v=[2,3,3,1,5,4,6,6,1]$, the construction gives:

$$
\begin{aligned}
\sigma_{v} & =\left[\begin{array}{llllllllll}
7 & 5 & 6 & 8 & 3 & 4 & 1 & 2 & 9 &
\end{array}\right] \\
v & =\left[\begin{array}{llllllllll}
2 & 3 & 3 & 1 & 5 & 4 & 6 & 6 & 1
\end{array}\right]
\end{aligned}
$$

We verify that $v \sigma_{v}^{-1}=[6,6,5,4,3,3,2,1,1]=v^{+}$.

The definition of $\sigma_{v}$ is compatible with the action of $\mathfrak{S}_{N}$ in the following sense:

\section{Proposition 2.3.}

$$
\begin{aligned}
& \text { 1. } \sigma_{v s_{i}}= \begin{cases}\sigma_{v} & \text { if } v=v s_{i}, \\
\sigma_{v} s_{i} & \text { otherwise. }\end{cases} \\
& \text { 2. If } v[i]=v[i+1] \text { then } \sigma_{v} s_{i}=s_{\sigma_{v}[i]} \sigma_{v} .
\end{aligned}
$$

This can be easily obtained from the construction.

Define the affine operation $\Psi$ acting on a vector by

$$
\left[v_{1}, \ldots, v_{N}\right] \Psi=\left[v_{2}, \ldots, v_{N}, v_{1}+1\right],
$$

and more generally let $\Psi^{\alpha}$ by

$$
\left[v_{1}, \ldots, v_{N}\right] \Psi^{\alpha}=\left[v_{2}, \ldots, v_{N}, v_{1}+\alpha\right] .
$$

Denote also by $\theta:=\Psi^{0}$ the circular permutation $[2, \ldots, N, 1]$.

Again, one can prove easily that the computation of $\sigma_{v}$ is compatible (in a certain sense) with the action of $\Psi$ : 


\section{Proposition 2.4.}

$$
\sigma_{v \Psi}=\sigma_{v} \theta
$$

Example 2.5. Consider $v=[2,3,3,2,5,4,6,6,1]$, one has

$$
\sigma_{v}=[7,5,6,8,3,4,1,2,9]
$$

and

$$
\sigma_{v} \theta=[5,6,8,3,4,1,2,9,7]
$$

But

$$
v^{\prime}:=v[2,3,4,5,6,7,8,9,1]=[3,3,2,5,4,6,6,1,2]
$$

and $\sigma_{v^{\prime}}=[5,6, \underline{7}, 3,4,1,2,9, \underline{8}]$; here an underlined integer means that there is a difference with the same position in $\sigma_{v} \theta$ and in $\sigma_{v^{\prime}}$. This is due to the fact that $v[1]$ is the first occurrence of 2 in $v$ while $v^{\prime}[9]$ is the last occurrence of 2 in $v^{\prime}$. Adding 1 to $v^{\prime}[9]$ one obtains

$$
v \Psi=[3,3,2,5,4,6,6,1,3] .
$$

The last occurrence of 2 becomes the last occurrence of 3 (that is the number of the first occurrence of 2 minus 1). Hence,

$$
\sigma_{v \Psi}=[5,6,8,3,4,1,2,9,7]=\sigma_{v} \theta .
$$

\subsection{Construction and basic properties of the graph}

Definition 2.6. A tableau of shape $\lambda$ is a filling with integers weakly increasing in each row and in each column. In the sequel row-strict means increasing in each row and column-strict means increasing in each column.

A reverse standard tableau (RST) is obtained by filling the shape $\lambda$ with integers $1, \ldots, N$ and with the conditions of strictly decreasing in the row and the column. We will denote by $\mathrm{Tab}_{\lambda}$, the set of the RST with shape $\lambda$.

Let $\tau$ be a RST, we define the vector of contents of $\tau$ as the vector $\mathrm{CT}_{\tau}$ such that $\mathrm{CT}_{\tau}[i]$ is the content of $i$ in $\tau$ (that is the number of the diagonal in which $i$ appears; the number of the main diagonal is 0 , and the numbers decrease from down to up). In other words, if $i$ appears in the box $[\mathrm{col}$, row $]$ then $\mathrm{CT}_{\tau}[i]=\mathrm{col}-$ row.

Example 2.7. Consider the tableau $\tau=\begin{array}{lll}2 & \\ 5 & 4\end{array}$, we obtain the vector of contents by labeling $\begin{array}{lll}6 & 3 & 1\end{array}$

the numbers of the diagonals $\begin{array}{rrrr}-2 & & & \\ -1 & 0 & \\ 0 & 1 & 2\end{array}$. So one obtains,

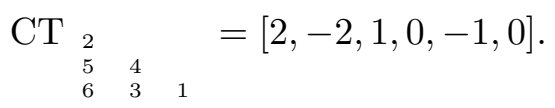

We construct a Yang-Baxter-type graph with vertices labeled by 4 -tuples $(\tau, \zeta, v, \sigma)$, where $\tau$ is a $\operatorname{RST}, \zeta$ is a vector of length $N$ with entries in $\mathbb{Z}[\alpha]$ ( $\zeta$ will be called the spectral vector), $v \in \mathbb{N}^{N}$ and $\sigma \in \mathfrak{S}_{N}$, as follows: First, consider a RST of shape $\lambda$ and write a vertex labeled by 
the 4-tuple $\left(\tau, \mathrm{CT}_{\tau}, 0^{N},[1, \ldots, N]\right)$. Now, we consider the action of the elementary transposition of $\mathfrak{S}_{N}$ on the 4 -tuple given by

$$
(\tau, \zeta, v, \sigma) s_{i}= \begin{cases}\left(\tau, \zeta s_{i}, v s_{i}, \sigma s_{i}\right) & \text { if } v[i+1] \neq v[i], \\ \left(\tau^{(\sigma[i], \sigma[i+1])}, \zeta s_{i}, v, \sigma\right) & \text { if } v[i]=v[i+1] \text { and } \tau^{(\sigma[i], \sigma[i+1])} \in \mathrm{Tab}_{\lambda} \\ (\tau, \zeta, v, \sigma) & \text { otherwise }\end{cases}
$$

where $\tau^{(i, j)}$ denotes the filling obtained by permuting the values $i$ and $j$ in $\tau$.

Consider also the affine action given by

$$
(\tau, \zeta, v, \sigma) \Psi=\left(\tau, \zeta \Psi^{\alpha}, v \Psi, \sigma[2, \ldots, N, 1]\right)=\left(\tau,\left[\zeta_{2}, \ldots,\right],\left[v_{2}, \ldots\right],\left[\sigma_{2}, \ldots\right]\right) .
$$

\section{Example 2.8.}

1.

$$
\begin{aligned}
\left(\begin{array}{l}
31 \\
542
\end{array},[1,0,2 \alpha, \alpha+2, \alpha-1],[0,0,2,1,1],[45123]\right) s_{2}= \\
\left(\begin{array}{l}
31 \\
542
\end{array},[1,2 \alpha, 0, \alpha+2, \alpha-1],[0,2,0,1,1],[41523]\right)
\end{aligned}
$$

2.

$$
\begin{aligned}
\left(\begin{array}{l}
31 \\
542
\end{array},[1,0,2 \alpha, \alpha+2, \alpha-1],[0,0,2,1,1],[45123]\right) s_{4}= \\
\left({ }_{543}^{21},[1,0,2 \alpha, \alpha-1, \alpha+2],[0,0,2,1,1],[45123]\right)
\end{aligned}
$$

3.

$$
\begin{aligned}
\left(\begin{array}{l}
31 \\
542
\end{array},[1,0,2 \alpha, \alpha+2, \alpha-1],[0,0,2,1,1],[45123]\right) s_{1}= \\
\left(\begin{array}{l}
31 \\
542
\end{array},[1,0,2 \alpha, \alpha+2, \alpha-1],[0,0,2,1,1],[45123]\right)
\end{aligned}
$$

4.

$$
\begin{aligned}
&\left(\begin{array}{l}
31 \\
542
\end{array},[1,0,2 \alpha, \alpha+2, \alpha-1],[0,0,2,1,1],[45123]\right) \Psi= \\
&\left(\begin{array}{l}
31 \\
542
\end{array},[0,2 \alpha, \alpha+2, \alpha-1, \alpha+1],[0,2,1,1,1],[51234]\right)
\end{aligned}
$$

Definition 2.9. If $\lambda$ is a partition, denote by $\tau_{\lambda}$ the tableau obtained by filling the shape $\lambda$ from bottom to top and left to right by the integers $\{1, \ldots, N\}$ in the decreasing order.

The graph $G_{\lambda}$ is an infinite directed graph constructed from the 4-tuple

$$
\left(\tau_{\lambda}, \mathrm{CT}_{\tau_{\lambda}},\left[0^{N}\right],[1,2, \ldots, N]\right),
$$

called the root and adding vertices and edges following the rules

1. We add an arrow labeled by $s_{i}$ from the vertex $(\tau, \zeta, v, \sigma)$ to $\left(\tau^{\prime}, \zeta^{\prime}, v^{\prime}, \sigma^{\prime}\right)$ if $(\tau, \zeta, v, \sigma) s_{i}=$ $\left(\tau^{\prime}, \zeta^{\prime}, v^{\prime}, \sigma^{\prime}\right)$ and $v[i]<v[i+1]$ or $v[i]=v[i+1]$ and $\tau$ is obtained from $\tau^{\prime}$ by interchanging the position of two integers $k<\ell$ such that $k$ is at the south-east of $\ell$ (i.e. $\operatorname{CT}_{\tau}(k) \geq$ $\left.\mathrm{CT}_{\tau}(\ell)+2\right)$.

2. We add an arrow labeled by $\Psi$ from the vertex $(\tau, \zeta, v, \sigma)$ to $\left(\tau^{\prime}, \zeta^{\prime}, v^{\prime}, \sigma^{\prime}\right)$ if $(\tau, \zeta, v, \sigma) \Psi=$ $\left(\tau^{\prime}, \zeta^{\prime}, v^{\prime}, \sigma^{\prime}\right)$.

3. We add an arrow $s_{i}$ from the vertex $(\tau, \zeta, v, \sigma)$ to $\varnothing$ if $(\tau, \zeta, v, \sigma) s_{i}=(\tau, \zeta, v, \sigma)$.

An arrow of the form

$$
(\tau, \zeta, v, \sigma) \quad s_{i} \text { or } \Psi \longrightarrow\left(\tau, \zeta^{\prime}, v^{\prime}, \sigma^{\prime}\right)
$$

will be called a step. The other arrows will be called jumps, and in particular an arrow

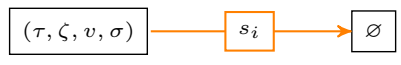

will be called a fall; the other jumps will be called correct jumps.

As usual a path is a sequence of consecutive arrows in $G_{\lambda}$ starting from the root and is denoted by the sequence if the labels of its arrows. Two paths $\mathfrak{P}_{1}=\left(a_{1}, \ldots, a_{k}\right)$ and $\mathfrak{P}_{2}=\left(b_{1}, \ldots, b_{\ell}\right)$ are said to be equivalent (denoted by $\mathfrak{P}_{1} \equiv \mathfrak{P}_{2}$ ) if they lead to the same vertex. 
We remark that from Proposition 2.3, in the case $v[i]=v[i+1]$, the part 1 of Definition 2.9 is equivalent to the following statement: $\tau^{\prime}$ is obtained from $\tau$ by interchanging $\sigma_{v}[i]$ and $\sigma_{v}[i+1]=$ $\sigma_{v}[i]+1$ where $\sigma_{v}[i]$ is to the south-east of $\sigma_{v}[i]+1$, that is, $\mathrm{CT}_{v}\left[\sigma_{v}[i]\right]-\mathrm{CT}_{v}\left[\sigma_{v}[i]+1\right] \geq 2$.

Example 2.10. The following arrow is a correct jump

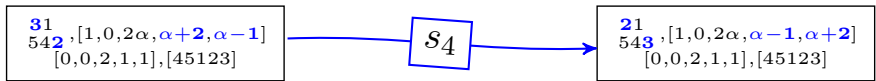

whilst

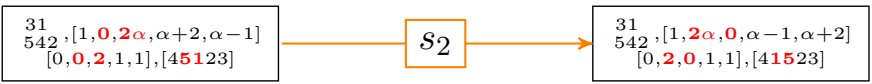

is a step.

The arrows

\begin{tabular}{|c|c|}
\hline $11,[1,0,2 \alpha, \alpha+2, \alpha-1]$ \\
$542,0,2,1,1],[45123]$
\end{tabular}$\longleftarrow S_{4}^{21},$\begin{tabular}{c}
$21,[1,0,2 \alpha, \alpha-1, \alpha+2]$ \\
$543,[0,0,2,1,1],[45123]$ \\
\hline
\end{tabular}

and

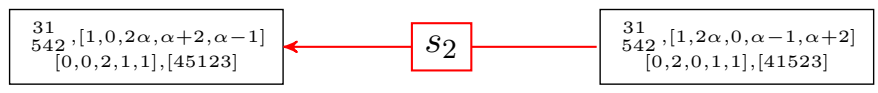

are not allowed.

Example 2.11. Consider the partition $\lambda=21$, the graph $G_{21}$ in Fig. 1 is obtained from the 4 -tuple $\left(\begin{array}{cc}2 \\ 3 & 1\end{array},(1,-1,0),(0,0,0), 1\right)$ by applying the rules of Definition 2.9. In Fig. 1, the steps are drawn in orange, the jumps in blue and the falls have been omitted.

For a reverse standard tableau $\tau$ of shape $\lambda$, a partition of $N$, let

$$
\operatorname{inv}(\tau)=\#\{(i, j): 1 \leq i<j \leq N, \operatorname{rw}(i, \tau)>\operatorname{rw}(j, \tau)\},
$$

where $\operatorname{rw}(i, \tau)$ is the row of $\tau$ containing $i$ (also we denote the column containing $i$ by $\operatorname{cl}(i, \tau)$ ). Then a correct jump from $\tau$ to $\tau^{\prime} \operatorname{implies} \operatorname{inv}\left(\tau^{\prime}\right)=\operatorname{inv}(\tau)+1$ (the entries $\sigma[i]$ and $\sigma[i+1]=\sigma[i]+1$ are interchanged in $\tau$ to produce $\tau^{\prime}$ ). Thus the number of correct jumps in a path from the root to $(\tau, \zeta, v, \sigma)$ equals $\operatorname{inv}(\tau)-\operatorname{inv}\left(\tau_{\lambda}\right)$.

So we consider the number of steps in a path from $0^{N}$ to $v ;{ }^{1}$ recall that one step links $v$ to $v^{\prime}$ where either $v[i]<v[i+1]$ and $v^{\prime}=v s_{i}$ or $v^{\prime}=v \Psi$.

For $x \in \mathbb{Z}($ or $\mathbb{R})$ let $\epsilon(x):=\frac{1}{2}(|x|+|x+1|-1)$, then $\epsilon(x)=x$ for $x \geq 0, \epsilon(x)=0$ for $-1 \leq x \leq 0$, and $\epsilon(x)=-x-1$ for $x \leq-1$.

There is a symmetry relation: $\epsilon(x)=\epsilon(-x-1)$.

Definition 2.12. For $v \in \mathbb{N}^{N}$ let $|v|:=\sum_{i=1}^{N} v[i]$ and set

$$
\mathrm{S}(v):=\sum_{1 \leq i<j \leq N} \epsilon(v[i]-v[j])
$$

The above formula can be written as

$$
\mathrm{S}(v)=\frac{1}{2} \sum_{1 \leq i<j \leq N}(|v[i]-v[j]|+|v[i]-v[j]+1|)-\frac{N(N-1)}{4} .
$$

\footnotetext{
${ }^{1}$ The other components of the label of the vertices are omitted here.
} 


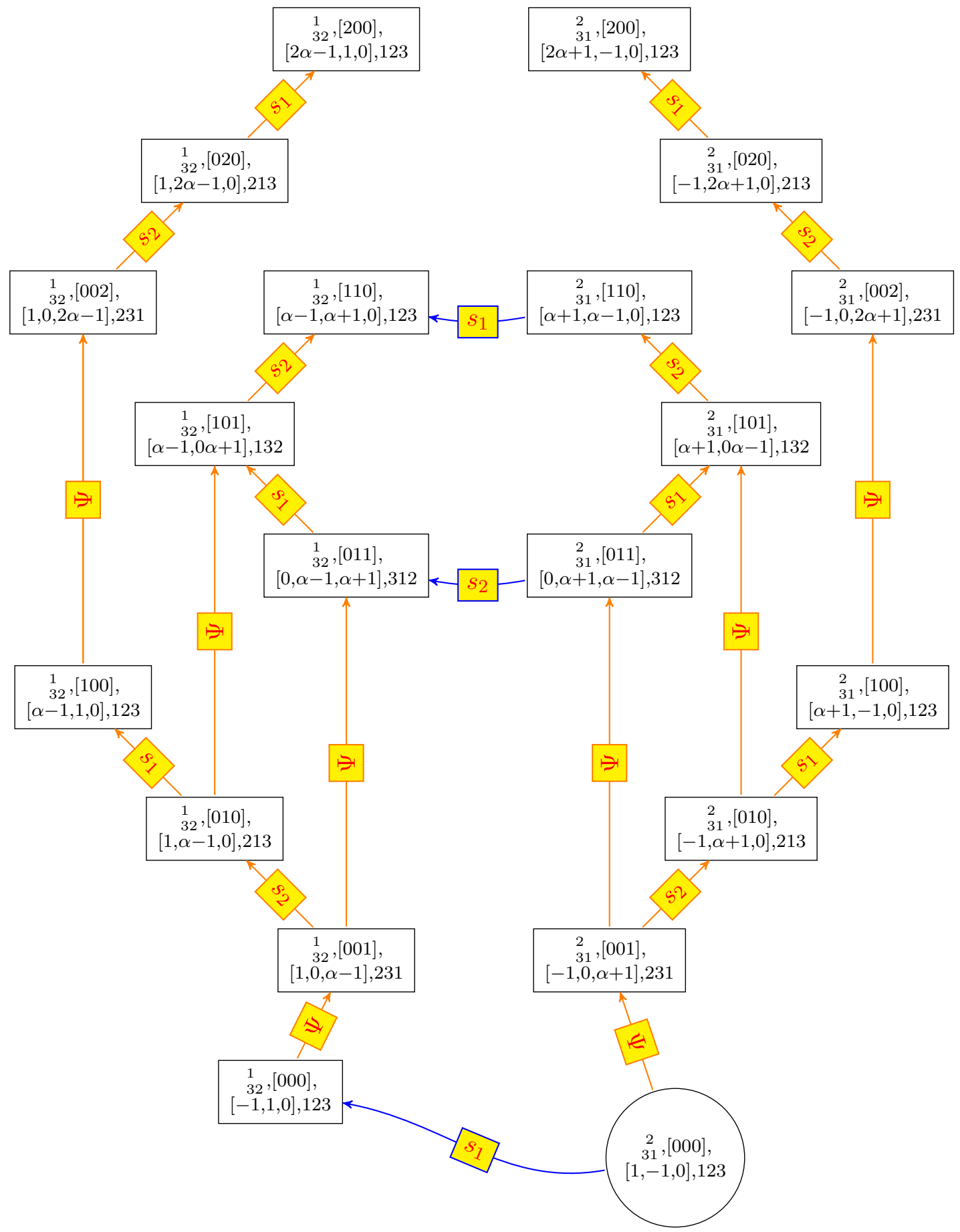

Figure 1. The first vertices of the graph $G_{21}$ where we omit to write the vertex $\varnothing$ and the associated arrows.

Proposition 2.13. The number of steps in any path joining $0^{N}$ to $v$ equals $|v|+\mathrm{S}(v)$.

Proof. The base point satisfies $\left|0^{N}\right|=0$ and $\left|\mathrm{S}\left(0^{N}\right)\right|=0$. Since jumps do not modify $v$, consider a step of the form $v^{\prime}=v s_{m}$, then $\left|v^{\prime}\right|=|v|, v[m+1]-v[m] \geq 1$ and $\mathrm{S}\left(v^{\prime}\right)-\mathrm{S}(v)$ involves only the pair $(m, m+1)$ in the sum over all pairs $(i, j), 1 \leq i<j \leq N$. Indeed

$$
\begin{aligned}
\mathrm{S}\left(v^{\prime}\right)-\mathrm{S}(v) & =\epsilon(v[m+1]-v[m])-\epsilon(v[m]-v[m+1]) \\
& =(v[m+1]-v[m])-(-v[m]+v[m+1]-1)=1 .
\end{aligned}
$$


It remains to show that $\mathrm{S}(v \Psi)=\mathrm{S}(v)$ (because $|v \Psi|=|v|+1)$. Note $(v \Psi)[N]=v[1]+1$. Then

$$
\begin{aligned}
\mathrm{S}(v)-\mathrm{S}(v \Psi) & =\sum_{j=2}^{N} \epsilon(v[1]-v[j])-\sum_{i=2}^{N} \epsilon(v[i]-v[1]-1) \\
& =\sum_{j=2}^{N} \epsilon(v[1]-v[j])-\epsilon(v[j]-v[1]-1)=0 .
\end{aligned}
$$

This completes the proof.

As a straightforward consequences, Proposition 2.13 implies

Corollary 2.14. All the paths joining two given vertices in $G_{\lambda}$ have the same length.

This suggests that some properties could be shown by induction on the common length of all the all the paths joining two given vertices.

For a given 4-tuple $(\tau, \zeta, v, \sigma)$ the values of $\zeta$ and $\sigma$ are determined by those of $\tau$ and $v$, as shown by the following proposition.

Proposition 2.15. If $(\tau, \zeta, v, \sigma)$ is a vertex in $G_{\lambda}$, then $\sigma=\sigma_{v}$ and $\zeta[i]=v_{i} \alpha+C T_{\tau}[\sigma[i]]$. We will set $\zeta_{v, \tau}:=\zeta$.

Proof. We prove the result by induction on the length $k$ of a path $\left(a_{1}, \ldots, a_{k}\right)$ (from Corollary 2.14 all the paths have the same length) from the root to $(\tau, \zeta, v, \sigma)$ and set

$$
\left(\tau^{\prime}, \zeta^{\prime}, v^{\prime}, \sigma^{\prime}\right)=\left(\tau_{\lambda}, \mathrm{CT}_{\tau_{\lambda}}, 0^{N},[1, \ldots, N]\right) a_{1} \cdots a_{k-1} .
$$

Suppose that $a_{k}=\Psi$ is the affine operation. More precisely, $\tau=\tau^{\prime}, \zeta=\zeta^{\prime} \Psi^{\alpha}, v=v^{\prime} \Psi$ and $\sigma=$ $\sigma^{\prime}[2, \ldots, N, 1]$. Using the induction hypothesis one has $\sigma^{\prime}=\sigma_{v^{\prime}}$ and $\zeta^{\prime}[i]=v^{\prime}[i] \alpha+\mathrm{CT}_{\tau}\left[\sigma_{v^{\prime}}[i]\right]$. Hence, Proposition 2.4 gives $\sigma=\sigma_{v^{\prime} \Psi}=\sigma_{v}$. Suppose that $i<N$ then

$$
\zeta[i]=\zeta^{\prime}[i+1]=v^{\prime}[i+1] \alpha+\mathrm{CT}_{\tau}\left[\sigma_{v^{\prime}}[i+1]\right]=v[i] \alpha+\mathrm{CT}_{\tau}\left[\sigma_{v}[i]\right] .
$$

If $i=N$ then again

$$
\zeta[i]=\zeta^{\prime}[1]+a=\left(v^{\prime}[1]+1\right) \alpha+\mathrm{CT}_{\tau}\left[\sigma_{v^{\prime}}[1]\right]=v[N] \alpha+\mathrm{CT}_{\tau}\left[\sigma_{v}[N]\right] .
$$

Suppose now that $a_{k}$ is not an affine operation. Using the induction hypothesis one has $\sigma^{\prime}=\sigma_{v^{\prime}}$ and $\zeta^{\prime}[j]=v^{\prime}[j] \alpha+\mathrm{CT}_{\tau}\left[\sigma_{v^{\prime}}[j]\right]$ for each $j$. If $a_{k}=s_{i}$ is a step then $\tau=\tau^{\prime}, \zeta=\zeta^{\prime} s_{i}, v=v^{\prime} s_{i}$ and $\sigma=\sigma^{\prime} s_{i}$. Hence, Proposition 2.3 gives $\sigma=\sigma_{v^{\prime} s_{i}}=\sigma_{v}$. If $j \neq i, i+1$ then one has $\zeta[j]=\zeta^{\prime}[j]$, $v[j]=v^{\prime}[j]$ and $\sigma[j]=\sigma^{\prime}[j]$, hence $\zeta[j]=v[j] \alpha+\mathrm{CT}_{\tau}\left[\sigma_{v}[j]\right]$. If $j=i$ then one has $\zeta[i]=\zeta^{\prime}[i+1]$, $v[i]=v^{\prime}[i+1]$ and $\sigma[i]=\sigma^{\prime}[i+1]$, and again the result is straightforward. And similarly when $j=i+1$ one finds the correct value for $\zeta[i+1]$.

Suppose now that $a_{k}=s_{i}$ is a jump. That is $\tau=\tau^{\prime(\sigma[i], \sigma[i+1])}, \zeta=\zeta^{\prime} s_{i}, v=v^{\prime}$ and $\sigma=\sigma^{\prime}$. Straightforwardly, $\sigma=\sigma_{v^{\prime}}=\sigma_{v}$ and if $j \neq i, i+1$ then

$$
\zeta[j]=\zeta^{\prime}[j]=v^{\prime}[j] \alpha+\mathrm{CT}_{\tau^{\prime}}\left[\sigma_{v^{\prime}}[j]\right]=v[j] \alpha+\mathrm{CT}_{\tau}\left[\sigma_{v}[j]\right]
$$

Suppose that $j=i$, since $a_{k}$ is a jump $v^{\prime}[i]=v^{\prime}[i+1]$ and

$$
\zeta[i]=\zeta^{\prime}[i+1]=v^{\prime}[i+1] \alpha+\mathrm{CT}_{\tau^{\prime}}\left[\sigma_{v^{\prime}}[i+1]\right]=v[i] \alpha+\mathrm{CT}_{\tau}\left[\sigma_{v}[i]\right] .
$$

Similarly, when $j=i+1$, one obtains the correct value for $\zeta[j]$. This ends the proof. 
Example 2.16. Consider the RST $\tau=\begin{array}{lll}7 & 4 & 1\end{array}$ and the vector $v=[6,2,4,2,2,3,1,4]$. $\begin{array}{llll}8 & 6 & 5 & 2\end{array}$

One has $\sigma_{v}=[1,5,2,6,7,4,8,3]$ and $\mathrm{CT}_{\tau}=[1,3,-2,0,2,1,-1,0]$ and then

$$
\zeta_{v, \tau}=[6 \alpha+1,2 \alpha+2,4 \alpha+3,2 \alpha+1,2 \alpha-1,3 \alpha, \alpha, 4 \alpha-2] .
$$

Hence, the 4-tuple

$$
\left(\begin{array}{cccc}
3 & & & \\
7 & 4 & 1 & ,[6 \alpha+1,2 \alpha+2,4 \alpha+3,2 \alpha+1,2 \alpha-1,3 \alpha, \alpha, 4 \alpha-2],[6,2,4,2,2,3,1,4],[1,5,2,6,7,4,8,3] \\
8 & 6 & 5 & 2
\end{array}\right)
$$

labels a vertex of $G_{431}$.

As a consequence,

Corollary 2.17. Let $(\tau, v)$ be a pair constituted with a $R S T \tau$ of shape $\lambda$ (a partition of $N$ ) and a vector $v \in \mathbb{N}^{N}$. Then there exists a unique vertex in $G_{\lambda}$ labeled by a 4-tuple of the form $(\tau, \zeta, v, \sigma)$. We will denote $\mathfrak{V}_{\tau, \zeta, v, \sigma}:=(\tau, v)$.

We point out that all the information can be retrieved from the spectral vector $\zeta$ - the coefficients of $\alpha$ give $v$, the rank function of $v$ gives $\sigma$, and the constants in the spectral vector give the content vector which does uniquely determine the RST $\tau$.

Definition 2.18. We define the subgraph $G_{\tau}$ as the graph obtained from $G_{\lambda}$ by erasing all the vertices labeled by RST other than $\tau$ and the associated arrows. Such a graph is connected.

Note that the graph $G_{\lambda}$ is the union of the graphs $G_{\tau}$ connected by jumps. Furthermore, if $G_{\tau}$ and $G_{\tau^{\prime}}$ are connected by a succession of jumps then there is no step from $G_{\tau^{\prime}}$ to $G_{\tau}$.

Example 2.19. In Fig. 1, the graph $G_{21}$ is constituted with the two graphs $G_{12}$ and $G_{31}$ connected by jumps (in blue).

\section{Vector-valued polynomials}

\subsection{About the Young seminormal representation of the symmetric group}

We consider the space $V_{\lambda}$ spanned by reverse tableaux of shape $\lambda$ and the (Young) action of the symmetric group as defined by Murphy ${ }^{2}$ in [17] by

$$
\tau s_{i}= \begin{cases}b_{\tau}[i] \tau & \text { if } b_{\tau}[i]^{2}=1 \\ b_{\tau}[i] \tau+\tau^{(i, i+1)} & \text { if } 0<b_{\tau}[i] \leq \frac{1}{2} \\ b_{\tau}[i] \tau+\left(1-b_{\tau}[i]^{2}\right) \tau^{(i, i+1)} & \text { otherwise }\end{cases}
$$

where $b_{\tau}[i]:=\frac{1}{\mathrm{CT}_{\tau}[i]-\mathrm{CT}_{\tau}[i+1]}$. Note that when $\left|b_{\tau}[i]\right|<1, \tau^{(i, i+1)}$ is always a reverse standard tableau when $\tau$ is a reverse standard tableau.

Murphy showed [17] that the RST are the simultaneous eigenfunctions of the Jucys-Murphy elements:

$$
\omega_{i}=\sum_{j=i+1}^{N} s_{i j}
$$

where $s_{i j}$ denotes the transposition exchanging $i$ and $j$. More precisely:

\footnotetext{
${ }^{2}$ The Young seminormal representation was defined in Young's last papers but himself apparently underestimated the importance of the construction. G. Murphy rediscovered it when reading the Jucys' paper [11]. See [18] for more details about the seminormal representation and its relation with the notions of Gelfand-Tsetlin basis.
} 


\section{Proposition 3.1.}

$$
\tau \omega_{i}=\mathrm{CT}_{\tau}[i] \tau .
$$

As usual, a polynomial representation for the Murphy action on the RST can be computed through the Yang-Baxter graph. We start from $\tau_{\lambda}$ and we construct the associated polynomial in the variables $t_{1}, \ldots, t_{N}$ :

$$
P_{\tau_{\lambda}}=\prod_{i} \prod_{\substack{k>l \\(i, k),(i, l) \in \lambda}}\left(t_{\tau_{\lambda}[i, k]}-t_{\tau_{\lambda}[i, l]}\right)
$$

where $\tau[i, j]$ denotes the integer belonging at the column $i$ and the row $j$ in $\tau$. Such a polynomial is a simultaneous eigenfunction of the Jucys-Murphy idempotents:

$$
P_{\tau_{\lambda}} \omega_{i}=\mathrm{CT}_{\tau_{\lambda}}[i] P_{\tau_{\lambda}} .
$$

Suppose that $P_{\tau}$ is the polynomial associated to $\tau$. Suppose also that $0<b_{\tau}[i]<1$. Hence, the polynomial $P_{\tau^{(i, i+1)}}$ is obtained from the polynomial $P_{\tau}$ by acting with $s_{i}-b_{\tau}[i]$ (with the standard action of the transposition $s_{i}$ on the variables $t_{j}$ ).

\section{Example 3.2.}

$$
\begin{aligned}
P_{43} & =P_{31}\left(s_{2}-\frac{1}{2}\right)=\left(t_{3}-t_{4}\right)\left(t_{1}-t_{2}\right)\left(s_{2}-\frac{1}{2}\right) \\
& =t_{1} t_{2}-\frac{1}{2} t_{4} t_{1}-\frac{1}{2} t_{3} t_{2}+t_{4} t_{3}-\frac{1}{2} t_{4} t_{2}-\frac{1}{2} t_{3} t_{1}
\end{aligned}
$$

Let us remark that in [13], Lascoux simplified the Young construction by having recourse to the covariant algebra $\left(\right.$ of $\left.\mathfrak{S}_{N}\right) \mathbb{C}\left[x_{1}, \ldots, x_{N}\right] / \mathfrak{S y m}_{+}$where $\mathfrak{S}_{\mathbf{y m}}$ is the ideal generated by symmetric functions without constant terms. Note that the covariant algebra is isomorphic to the regular representation $\mathbb{C}\left[\mathfrak{S}_{N}\right]$. In the aim to adapt his construction to our notations, we replace each polynomial with its dominant monomial represented by the vectors of its exponents. The vector associated to the root of the graph is the vector exponent of the leading monomial in the product of the Vandermonde determinants associated to each column and is obtained by putting the number of the row minus 1 in the corresponding entry.

Example 3.3. The vector associated to ${ }_{62}^{4}$ is $[010210]$.

In fact, the covariant algebra being isomorphic to the regular representation of $\mathfrak{S}_{N}$, the computation of the polynomials is completely encoded by the action of the symmetric group on the leading monomials, as shown in the following example. Observe that we do not replace the representation by the orbit of the leading monomial (since the space generated by the orbit is in general bigger), but we consider the projection which completely determines the elements.

Example 3.4. Consider the RST of shape 221, one has
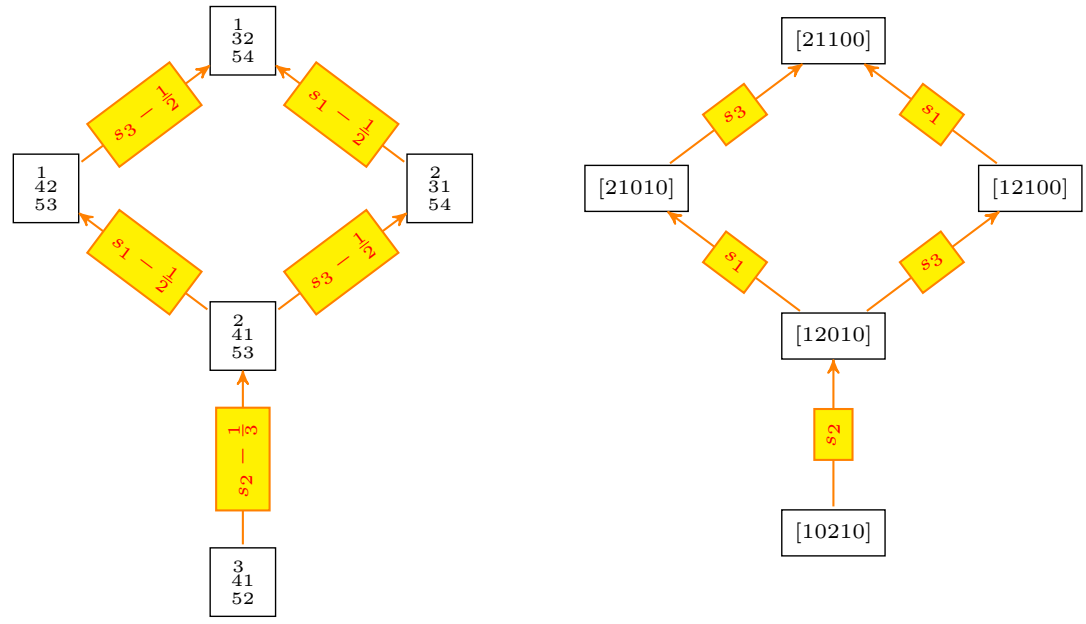
For instance, one has

$$
\begin{aligned}
P_{\substack{42 \\
5_{3}}}= & t_{1}^{2} t_{4} t_{2}+\frac{1}{2} t_{4} t_{2}^{2} t_{3}+\frac{1}{2} t_{2}^{2} t_{5} t_{1}-\frac{1}{2} t_{2}^{2} t_{5} t_{3}+\frac{1}{2} t_{4} t_{3}^{2} t_{1}+\frac{1}{2} t_{4}^{2} t_{1} t_{3}+t_{1}^{2} t_{5} t_{3}-t_{1}^{2} t_{5} t_{2}+\frac{1}{2} t_{5}^{2} t_{4} t_{3} \\
& +\frac{1}{2} t_{5} t_{4}^{2} t_{2}+\frac{1}{2} t_{5}^{2} t_{1} t_{2}-\frac{1}{2} t_{4} t_{2}^{2} t_{1}-\frac{1}{2} t_{5}^{2} t_{4} t_{2}+\frac{1}{2} t_{3}^{2} t_{5} t_{2}-\frac{1}{2} t_{4}^{2} t_{1} t_{2}-t_{1}^{2} t_{4} t_{3}-\frac{1}{2} t_{3}^{2} t_{5} t_{1} \\
& -\frac{1}{2} t_{5} t_{4}^{2} t_{3}-\frac{1}{2} t_{4} t_{3}^{2} t_{2}-\frac{1}{2} t_{5}^{2} t_{1} t_{3},
\end{aligned}
$$

whose leading monomial is $t_{1}^{2} t_{2} t_{4}$.

From the construction, the leading monomial of $P_{\tau}$ is the product of all the $t_{i}^{\mathrm{rw}(i, \tau)-1}$. For example, the leading monomial in $P_{\substack{512 \\ 932}}$ is $t_{1}^{2} t_{2} t_{3} t_{5}^{2} t_{7}$.

\subsection{Definition and dominance properties of vector-valued polynomials}

Consider the space

$$
M_{N}=\operatorname{span}_{\mathbb{C}}\left\{x_{1}^{v[1]} \cdots x_{N}^{v[N]} \otimes \tau: v \in \mathbb{N}^{N}, \tau \in \operatorname{Tab}_{\lambda}, \lambda \vdash N\right\},
$$

where $\operatorname{Tab}(N)$ denotes the set of the reverse standard tableaux on $\{1, \ldots, N\}$.

This space splits into a direct sum $M_{N}=\bigoplus_{\lambda \vdash N} M_{\lambda}$, where

$$
M_{\lambda}=\operatorname{span}_{\mathbb{C}}\left\{x_{1}^{v[1]} \cdots x_{N}^{v[N]} \otimes \tau \mid v \in \mathbb{N}^{N}, \tau \in \operatorname{Tab}_{\lambda}\right\} .
$$

The algebra $\mathbb{C}\left[\mathfrak{S}_{N}\right] \otimes \mathbb{C}\left[\mathfrak{S}_{N}\right]$ acts on these spaces by commuting the vector of the powers on the variables on the left component and the action on the tableaux defined by Murphy (equation (3.1)) on the right component.

\section{Example 3.5.}

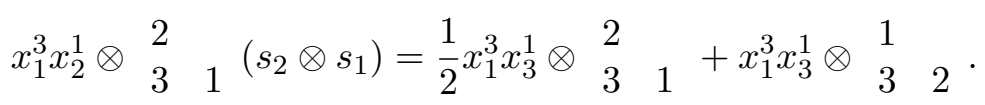

For simplicity we will denote $x^{v}=x_{1}^{v[1]} \cdots x_{N}^{v[N]}$ and $x^{v, \tau}:=x^{v} \otimes \tau \sigma_{v}$. By abuse of language $x^{v, \tau}$ will be referred to as a polynomial. Note that the space $M_{\lambda}$ is spanned by the set of polynomials

$$
\mathfrak{M}_{\lambda}:=\left\{x^{v, \tau}: v \in \mathbb{N}^{N}, \tau \in \operatorname{Tab}_{\lambda}\right\},
$$

which can be naturally endowed with the order $\triangleleft$ defined by

$$
x^{v, \tau} \tau \triangleleft x^{v^{\prime}, \tau^{\prime}} \text { iff } v \triangleleft v^{\prime},
$$

with $v \triangleleft v^{\prime}$ means that $v^{+} \prec v^{\prime+}$ or $v^{+}=v^{\prime+}$ and $v \prec v^{\prime}$, where $\prec$ denotes the classical dominance order on vectors:

$$
v \preceq v^{\prime} \quad \text { iff } \quad \forall i, v[1]+\cdots+v[i] \leq v^{\prime}[1]+\cdots+v^{\prime}[i] .
$$

\section{Example 3.6.}

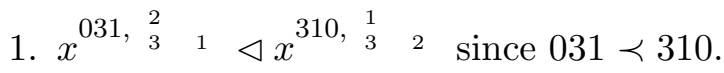

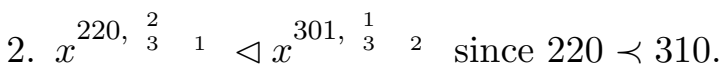

3. The polynomials $x^{031,{ }_{3}^{2}}{ }_{1}$ and $x^{031,{ }_{3}^{1}}{ }_{2}$ are not comparable. 
The partial order $\unlhd$ will provide us a relevant dominance notion.

Definition 3.7. The monomial $x^{v, \tau}$ is the leading monomial of a polynomial $P$ if and only if $P$ can be written as

$$
P=\alpha_{v} x^{v, \tau}+\sum_{x^{v^{\prime}, \tau^{\prime} \triangleleft x^{v, \tau}}} \alpha_{v^{\prime}, \tau^{\prime}} x^{v^{\prime}, \tau^{\prime}}
$$

with $\alpha_{v} \neq 0$.

As in [9], we define $\Psi:=(\theta \otimes \theta) x_{N}$, with $\theta=s_{1} s_{2} \cdots s_{N-1}$. The following proposition describes the transformation properties of leading monomials with respect to the $s_{i}$ and $\Psi$.

Proposition 3.8. Suppose that $x^{v, \tau}$ is the leading monomial in $P$ then

1. If $v[i]<v[i+1]$ then $x^{v s_{i}, \tau}$ is the leading monomial in $P\left(s_{i} \otimes s_{i}\right)$. Its leading monomial is $x^{\tilde{v}} \otimes\left(\tau \cdot s_{i j}\right)$, where $x^{\tilde{v}}$ is the dominant term in $\partial_{i j} x^{\tilde{v}}$.

2. $x^{v \Psi, \tau}$ is the leading monomial in $P \Psi$.

\subsection{Dunkl and Cherednik-Dunkl operators for vector-valued polynomials}

We define the Dunkl operators

$$
\mathfrak{D}_{i}:=\frac{\partial}{\partial x_{i}} \otimes 1+\frac{1}{\alpha} \sum_{i \neq j} \partial_{i j} \otimes s_{i j},
$$

where $s_{i j}$ denotes the transposition which exchanges $i$ and $j$ and

$$
\partial_{i j}=\left(1-s_{i j}\right) \frac{1}{x_{i}-x_{j}}
$$

is the divided difference.

This definition is the same as in [4], but our operators act on their left. One has

Lemma 3.9. If $\mathfrak{D}_{i}$ denotes the Dunkl operator, one has

$$
\left(s_{i} \otimes s_{i}\right) \mathfrak{D}_{i}=\mathfrak{D}_{i+1}\left(s_{i} \otimes s_{i}\right) .
$$

Proof. Straightforward from the definition of $\mathfrak{D}_{i}$ and the equalities

$$
s_{i} s_{i j}=s_{i+1, j} s_{i}, \quad s_{i} \partial_{i j}=\partial_{i+1 j} s_{i} \quad \text { and } \quad s_{i} \frac{\partial}{\partial x_{i}}=\frac{\partial}{\partial x_{i+1}} s_{i} .
$$

The Cherednik-Dunkl operators are pairwise commuting operators defined by [4]

$$
\mathfrak{U}_{i}:=x_{i} \mathfrak{D}_{i}-\frac{1}{\alpha} \sum_{j=1}^{i-1} s_{i, j} \otimes s_{i, j} .
$$

We do not repeat the proof of the commutation $\left[\mathfrak{U}_{i}, \mathfrak{U}_{j}\right]=0$ which can be found in [4]. But, as we will see in the next section, this property is not used to prove the existence of the vector-valued Jack polynomials.

One has

Lemma 3.10.

1. $\left(s_{i} \otimes s_{i}\right) \mathfrak{U}_{i}=\mathfrak{U}_{i+1}\left(s_{i} \otimes s_{i}\right)+\frac{1}{\alpha}$. 
2. $\left(s_{i} \otimes s_{i}\right) \mathfrak{U}_{j}=\mathfrak{U}_{j}\left(s_{i} \otimes s_{i}\right), j \neq i, i+1$.

3. $\left(s_{i} \otimes s_{i}\right) \mathfrak{U}_{i+1}=\mathfrak{U}_{i}\left(s_{i} \otimes s_{i}\right)-\frac{1}{\alpha}$.

Proof. The three identities are of the same type. We prove only the first one which follows from the equalities

$$
\begin{aligned}
\left(s_{i} \otimes s_{i}\right) \mathfrak{U}_{i} & =\left(s_{i} \otimes s_{i}\right) x_{i} \mathfrak{D}_{i}-\frac{1}{\alpha} \sum_{j=1}^{i-1} s_{i, j} \otimes s_{i, j} \\
& =\left(x_{i+1} \mathfrak{D}_{i+1}-\frac{1}{\alpha} \sum_{j=1}^{i-1} s_{i+1, j} \otimes s_{i+1, j}\right)\left(s_{i} \otimes s_{i}\right)=\mathfrak{U}_{i+1}\left(s_{i} \otimes s_{i}\right)+\frac{1}{\alpha} .
\end{aligned}
$$

The affine operator $\Psi$ has the following commutation properties with the Dunkl operators:

\section{Lemma 3.11.}

1. $\mathfrak{D}_{i+1} \Psi=\Psi \mathfrak{D}_{i}+(\theta \otimes \theta)\left(s_{i, N} \otimes s_{i, N}\right), i<1$.

2. $\mathfrak{D}_{1} \Psi=\Psi \mathfrak{D}_{N}+(\theta \otimes \theta)\left(\sum_{j=1}^{N-1}\left(s_{N, j} \otimes s_{N, j}\right)-1\right)$.

As a consequence, one finds.

\section{Lemma 3.12.}

$$
\Psi \mathfrak{U}_{i}=\mathfrak{U}_{i+1} \Psi, \quad i \neq N \quad \text { and } \quad \Psi \mathfrak{U}_{N}=\left(\mathfrak{U}_{1}+1\right) \Psi .
$$

The action on the RST is given by

\section{Lemma 3.13.}

$$
(1 \otimes \tau) \mathfrak{U}_{i}=\left(1+\frac{1}{\alpha} \mathrm{CT}_{\tau}[i]\right)(1 \otimes \tau)
$$

Proof. One has

$$
(1 \otimes \tau) \mathfrak{U}_{i}=(1 \otimes \tau) x_{i} \mathfrak{D}_{i}-\frac{1}{\alpha} \sum_{j=1}^{i-1}(1 \otimes \tau)\left(s_{i, j} \otimes s_{i, j}\right)=(1 \otimes \tau)\left(1+\frac{1}{\alpha} 1 \otimes \omega_{i}\right),
$$

where $\omega_{i}:=\sum_{j=i+1}^{N}(i j)$ denotes a Jucys-Murphy element. Since the RST are eigenfunctions of the Jucys-Murphy elements and the associated eigenvalues are given by the contents, the lemma follows.

For convenience, define $\tilde{\xi}_{i}:=\alpha \mathfrak{U}_{i}-\alpha$. From the preceding lemmas, one obtains

\section{Proposition 3.14.}

$$
\begin{aligned}
& \left(s_{i} \otimes s_{i}\right) \tilde{\xi}_{i}=\tilde{\xi}_{i+1}\left(s_{i} \otimes s_{i}\right)+1, \\
& \left(s_{i} \otimes s_{i}\right) \tilde{\xi}_{i+1}=\tilde{\xi}_{i}\left(s_{i} \otimes s_{i}\right)-1, \\
& \left(s_{i} \otimes s_{i}\right) \tilde{\xi}_{j}=\tilde{\xi}_{j}\left(s_{i} \otimes s_{i}\right), \quad j \neq i, i+1, \\
& \Psi \tilde{\xi}_{i}=\tilde{\xi}_{i+1} \Psi, \quad i \neq N, \\
& \Psi \tilde{\xi}_{N}=\left(\tilde{\xi}_{1}+\alpha\right) \Psi .
\end{aligned}
$$




\section{Nonsymmetric vector-valued Jack polynomials}

In this section we recover the construction, due to one of the authors [4], of a basis of vectorvalued polynomials $J_{v, \tau}$. This construction belongs to a large family of vector-valued Jack polynomials associated to the complex reflection groups $G(r, 1, n)$ defined by Griffeth [8]. We will denote by $\zeta_{v, \tau}$ their associated spectral vectors. We will see also that many properties of this basis can be deduced from the Yang-Baxter structure.

\subsection{Yang-Baxter construction associated to $G_{\lambda}$}

Let $\lambda$ be a partition and $G_{\lambda}$ be the associated graph. We construct the set of polynomials $\left(J_{\mathfrak{P}}\right)_{\mathfrak{P} \text { path in } G_{\lambda}}$ using the following recursive rules:

1. $J_{[]}:=\left(1 \otimes \tau_{\lambda}\right)$.

2. If $\mathfrak{P}=\left[a_{1}, \ldots, a_{k-1}, s_{i}\right]$ then

$$
J_{\mathfrak{P}}:=J_{\left[a_{1}, \ldots, a_{k-1}\right]}\left(s_{i} \otimes s_{i}+\frac{1}{\zeta[i+1]-\zeta[i]}\right),
$$

where the vector $\zeta$ is defined by

$$
\left(\tau_{\lambda}, \mathrm{CT}_{\tau_{\lambda}}, 0^{N},[1,2, \ldots, N]\right) a_{1} \ldots a_{k-1}=(\tau, \zeta, v, \sigma) .
$$

3. If $\mathfrak{P}=\left[a_{1}, \ldots, a_{k-1}, \Psi\right]$ then

$$
J_{\mathfrak{P}}=J_{\left[a_{1}, \ldots, a_{k-1}\right]} \Psi \text {. }
$$

One has the following theorem.

Theorem 4.1. Let $\mathfrak{P}=\left[a_{0}, \ldots, a_{k}\right]$ be a path in $G_{\lambda}$ from the root to $(\tau, \zeta, v, \sigma)$. The polynomial $J_{\mathfrak{P}}$ is a simultaneous eigenfunctions of the operators $\tilde{\xi}_{i}$ whose leading monomial is $x^{v, \tau}$. Furthermore, the eigenvalues of $\tilde{\xi}_{i}$ associated to $J_{\mathfrak{P}}$ are equal to $\zeta[i]$.

Consequently $J_{\mathfrak{P}}$ does not depend on the path, but only on the end point $(\tau, \zeta, v, \sigma)$, and will be denoted by $J_{v, \tau}$. The family $\left(J_{v, \tau}\right)_{v, \tau}$ forms a basis of $M_{\lambda}$ of simultaneous eigenfunctions of the Cherednik operators.

Furthermore, if $\mathfrak{P}$ leads to $\varnothing$ then $J_{\mathfrak{P}}=0$.

Proof. We will prove the result by induction on the length $k$. If $k=0$ then the result follows from Proposition 3.13. Suppose now that $k>0$ and let

$$
\left(\tau^{\prime}, \zeta^{\prime}, v^{\prime}, \sigma_{v^{\prime}}\right)=\left(\tau_{\lambda}, \mathrm{CT}_{\tau_{\lambda}}, 0^{N},[1, \ldots, N]\right) a_{1} \cdots a_{k-1} .
$$

By induction, $J_{\left[a_{1}, \ldots, a_{k-1}\right]}$ is a simultaneous eigenfunctions of the operators $\tilde{\xi}_{i}$ such that the associated vector of eigenvalues is given by

$$
J_{\left[a_{1}, \ldots, a_{k-1}\right]} \tilde{\xi}_{i}=\zeta^{\prime}[i] J_{\left[a_{1}, \ldots, a_{k-1}\right]}
$$

and the leading monomial is $x^{v^{\prime}, \tau^{\prime}}$.

If $a_{k}=\Psi$ is an affine arrow, then $\tau=\tau^{\prime}, \zeta=\zeta^{\prime} \cdot \Psi^{\alpha}, v=v^{\prime} \Psi, \sigma_{v}=\sigma_{v^{\prime}}[2, \ldots, N, 1]$ and $J_{\mathfrak{P}}=J_{\left[a_{1}, \ldots, a_{k-1}\right]} \Psi$. If $i \neq N$

$$
J_{\mathfrak{P}} \tilde{\xi}_{i}=J_{\left[a_{1}, \ldots, a_{k-1}\right]} \Psi \tilde{\xi}_{i}=J_{\left[a_{1}, \ldots, a_{k-1}\right]} \tilde{\xi}_{i+1} \Psi=\zeta^{\prime}[i+1] J_{\mathfrak{P}}=\zeta[i] J_{\mathfrak{P}} .
$$


If $i=N$ then,

$$
\left.J_{\mathfrak{P}} \tilde{\xi}_{N}=J_{\left[a_{1}, \ldots, a_{k-1}\right]} \Psi \tilde{\xi}_{N}=J_{\left[a_{1}, \ldots, a_{k-1}\right]} \tilde{(} \xi_{1}+\alpha\right) \Psi=\left(\zeta^{\prime}[1]+\alpha\right) J_{\mathfrak{P}}=\zeta[N] J_{\mathfrak{P}} .
$$

The leading monomial is a consequence of Proposition 3.8.

Suppose now that $a_{k}=s_{i}$ is a non affine arrow, then $\zeta=\zeta^{\prime} s_{i}, v=v^{\prime} s_{i}$ and

$$
J_{\mathfrak{P}}=J_{\left[a_{1}, \ldots, a_{k-1}\right]}\left(s_{i} \otimes s_{i}+\frac{1}{\zeta^{\prime}[i+1]-\zeta^{\prime}[i]}\right) .
$$

If $j \neq i, i+1$ then

$$
\begin{aligned}
J_{\mathfrak{P}} \tilde{\xi}_{j} & =J_{\left[a_{1}, \ldots, a_{k-1}\right]}\left(s_{i} \otimes s_{i}+\frac{1}{\zeta^{\prime}[i+1]-\zeta^{\prime}[i]}\right) \tilde{\xi}_{j} \\
& =J_{\left[a_{1}, \ldots, a_{k-1}\right]} \tilde{\xi}_{j}\left(s_{i} \otimes s_{i}+\frac{1}{\zeta^{\prime}[i+1]-\zeta^{\prime}[i]}\right)=\zeta^{\prime}[j] J_{\mathfrak{P}}=\zeta[j] J_{\mathfrak{P}} .
\end{aligned}
$$

If $j=i$ then

$$
\begin{aligned}
J_{\mathfrak{P}} \tilde{\xi}_{i} & =J_{\left[a_{1}, \ldots, a_{k-1}\right]}\left(s_{i} \otimes s_{i}+\frac{1}{\zeta^{\prime}[i+1]-\zeta^{\prime}[i]}\right) \tilde{\xi}_{i} \\
& =J_{\left[a_{1}, \ldots, a_{k-1}\right]}\left(\tilde{\xi}_{i+1}\left(s_{i} \otimes s_{i}\right)+1+\tilde{\xi}_{i} \frac{1}{\zeta^{\prime}[i+1]-\zeta^{\prime}[i]}\right) \\
& =J_{\left[a_{1}, \ldots, a_{k-1}\right]}\left(\zeta^{\prime}[i+1]\left(s_{i} \otimes s_{i}\right)+1+\frac{\zeta^{\prime}[i]}{\zeta^{\prime}[i+1]-\zeta^{\prime}[i]}\right) \\
& =\zeta^{\prime}[i+1] J_{\left[a_{1}, \ldots, a_{k-1}\right]}\left(s_{i} \otimes s_{i}+\frac{1}{\zeta^{\prime}[i+1]-\zeta^{\prime}[i]}\right)=\zeta[i] J_{\mathfrak{P}} .
\end{aligned}
$$

If $j=i+1$ then

$$
\begin{aligned}
J_{\mathfrak{P}} \tilde{\xi}_{i+1} & =J_{\left[a_{1}, \ldots, a_{k-1}\right]}\left(s_{i} \otimes s_{i}+\frac{1}{\zeta^{\prime}[i+1]-\zeta^{\prime}[i]}\right) \tilde{\xi}_{i} \\
& =J_{\left[a_{1}, \ldots, a_{k-1}\right]}\left(\tilde{\xi}_{i}\left(s_{i} \otimes s_{i}\right)-1+\tilde{\xi}_{i+1} \frac{1}{\zeta^{\prime}[i+1]-\zeta^{\prime}[i]}\right) \\
& =J_{\left[a_{1}, \ldots, a_{k-1}\right]}\left(\zeta^{\prime}[i]\left(s_{i} \otimes s_{i}\right)-1+\frac{\zeta^{\prime}[i+1]}{\zeta^{\prime}[i+1]-\zeta^{\prime}[i]}\right) \\
& =\zeta^{\prime}[i] J_{\left[a_{1}, \ldots, a_{k-1}\right]}\left(s_{i} \otimes s_{i}+\frac{1}{\zeta^{\prime}[i+1]-\zeta^{\prime}[i]}\right)=\zeta[i+1] J_{\mathfrak{P}} .
\end{aligned}
$$

Let us examine the leading monomials. First, suppose that $a_{k}=s_{i}$ is a step then $\tau=\tau^{\prime}$ and $\sigma_{v}=\sigma_{v^{\prime}} s_{i}$. From Proposition 3.8, the leading monomial in $J_{\mathfrak{P}}$ equals the leading term in $x^{v^{\prime} \tau^{\prime}}\left(s_{i} \otimes s_{i}+\frac{1}{\zeta^{\prime}[i+1]-\zeta^{\prime}[i]}\right)$ that is $x^{v^{\prime} s_{i}} \otimes\left(\tau^{\prime} \sigma_{v^{\prime}} s_{i}\right)=x^{v, \tau}$.

Suppose that $a_{k}=s_{i}$ is not a step and set $Q:=x^{v^{\prime}, \tau^{\prime}}\left(s_{i} \otimes s_{i}+\frac{1}{\left.\zeta^{\prime}[i+1]-\zeta^{\prime} \mid i\right]}\right)$. One has

$$
J_{\mathfrak{P}}=Q+\sum_{x^{v^{\prime \prime}, \tau^{\prime \prime}} \triangleleft x^{v, \tau}} \alpha_{v^{\prime \prime}, \tau^{\prime \prime}} x^{v^{\prime \prime}, \tau^{\prime \prime}} .
$$

If $a_{k}=s_{i}$ is a jump then $\tau=\tau^{\prime\left(\sigma_{v^{\prime}}[i], \sigma_{v^{\prime}}[i]+1\right)}$ and $\sigma_{v}=\sigma_{v}^{\prime}$. But

$$
Q=x^{v^{\prime} s_{i}} \otimes\left(\tau^{\prime} \sigma_{v^{\prime}} s_{i}\right)+\frac{1}{\zeta^{\prime}[i+1]-\zeta^{\prime}[i]} x^{v^{\prime}} \otimes\left(\tau^{\prime} \sigma_{v^{\prime}}\right)
$$




$$
\begin{aligned}
& =x^{v} \otimes\left(\tau^{\prime} s_{\sigma_{v^{\prime}}[i]} \sigma_{v^{\prime}}\right)+\frac{1}{\zeta^{\prime}[i+1]-\zeta^{\prime}[i]} x^{v^{\prime}} \otimes\left(\tau^{\prime} \sigma_{v^{\prime}}\right) \\
& =x^{v} \otimes\left(\tau \sigma_{v}\right)+\left(b_{\tau}^{\prime}\left[\sigma_{v^{\prime}}[i]\right]+\frac{1}{\zeta^{\prime}[i+1]-\zeta^{\prime}[i]}\right) x^{v^{\prime}} \otimes\left(\tau^{\prime} \sigma_{v^{\prime}}\right) \\
& =x^{v, \tau}+\left(b_{\tau}^{\prime}\left[\sigma_{v^{\prime}}[i]\right]+\frac{1}{\zeta^{\prime}[i+1]-\zeta^{\prime}[i]}\right) x^{v^{\prime}, \tau^{\prime}} .
\end{aligned}
$$

But $\zeta^{\prime}[i]=\mathrm{CT}_{\tau^{\prime}}\left[\sigma_{v^{\prime}}[i]\right]$ and $\zeta^{\prime}[i+1]=\mathrm{CT}_{\tau^{\prime}}\left[\sigma_{v^{\prime}}[i+1]\right]=\mathrm{CT}_{{\tau^{\prime}}^{\prime}}\left[\sigma_{v^{\prime}}[i]+1\right]$, hence $b_{\tau^{\prime}}\left[\sigma_{{v^{\prime}}^{\prime}}[i]\right]=$ $-\frac{1}{\zeta^{\prime}[i+1]-\zeta^{\prime}[i]}$. And the leading monomial is $Q=x^{v, \tau}$ as expected.

This proves the first part of the theorem and that the family $\left(J_{v, \tau}\right)_{v, \tau}$ forms a basis of $M_{\lambda}$ of simultaneous eigenfunctions of the Cherednik operators.

Finally, if $a_{k}=s_{i}$ is a fall, $Q$ is proportional to $x^{v^{\prime}} \otimes\left(\tau^{\prime} \sigma_{v^{\prime}}\right)$ and then $J_{\mathfrak{P}}$ is proportional to $J_{\left[a_{1}, \ldots, a_{k-1}\right]}$. But clearly, the two polynomials are eigenfunction of the Cherednik operators with different eigenvalues from the cases $j=i$ and $j=i+1$. This proves that $J_{\mathfrak{P}}=0$.

As a consequence, we will consider the family of polynomials $\left(J_{v, \tau}\right)_{v, \tau}$ indexed by pairs $(v, \tau)$ where $v \in \mathbb{N}^{N}$ is a weight and $\tau$ is a tableau.

Example 4.2. For $\lambda=21$, the first polynomials $J_{v, \tau}$ are displayed in Fig. 2. The spectral vectors can be read on Fig. 1.

Note that if $\left[a_{1}, \ldots, a_{k-1}\right]$ leads to a vertex other than $\varnothing$ and $\left[a_{1}, \ldots, a_{k-1}, s_{i}\right]$ leads to $\varnothing$, the last part of Theorem 4.1 implies that $J_{\left[a_{1}, \ldots, a_{k-1}\right]}$ is symmetric or antisymmetric under the action of $s_{i} \otimes s_{i}$.

The recursive rules of this section first appeared in [8]. The Lemma 5.3 and the Yang-Baxter graph constitute essentially what Griffeth called calibration graph in that paper.

\subsection{Partial Yang-Baxter-type construction associated to $G_{\tau}$}

To compute an expression for a polynomial $J_{v, \tau}$ it suffices to find the good path in the subgraph $G_{\tau}$ as shown by the following examples.

Example 4.3. Consider $\tau=\begin{aligned} & 1 \\ & 3\end{aligned} 2$, Fig. 3 explains how to obtains the values of $J_{v,{ }_{32}}$ from the graph $G_{32}$.

Example 4.4. For the trivial representation (i.e., $\lambda$ has a single part), note that the Cherednik operators (in [14]) have the same eigenspaces as the Cherednik-Dunkl operators $\mathfrak{U}_{i}$ (in [4]). In the notations of [14], $\xi_{i}$ reads

$$
\xi_{i}=\alpha x_{i} \frac{\partial}{\partial x_{i}}+\sum_{\substack{j=1 \\ j \neq i}}^{N} \bar{\pi}_{i j}+(1-i),
$$

where

$$
\bar{\pi}_{i j}= \begin{cases}x_{i} \partial_{i j} & \text { if } j<i \\ x_{j} \partial_{i j} & \text { if } i<j\end{cases}
$$

where $\partial_{i j}$ denotes the divided difference on the variables $x_{i}$ and $x_{j}$. Noting that $x_{i} \partial_{i j}=\partial_{i j} x_{i}-1$, $x_{j} \partial_{i j}=\partial_{i j} x_{i}-(i j)$ and $x_{i} \frac{\partial}{\partial x_{i}}=\frac{\partial}{\partial x_{i}} x_{i}-1$, one finds

$$
\xi_{i}=\alpha \mathfrak{U}_{i}-(\alpha+N-1)=\tilde{\xi}_{i}-(N-1) .
$$




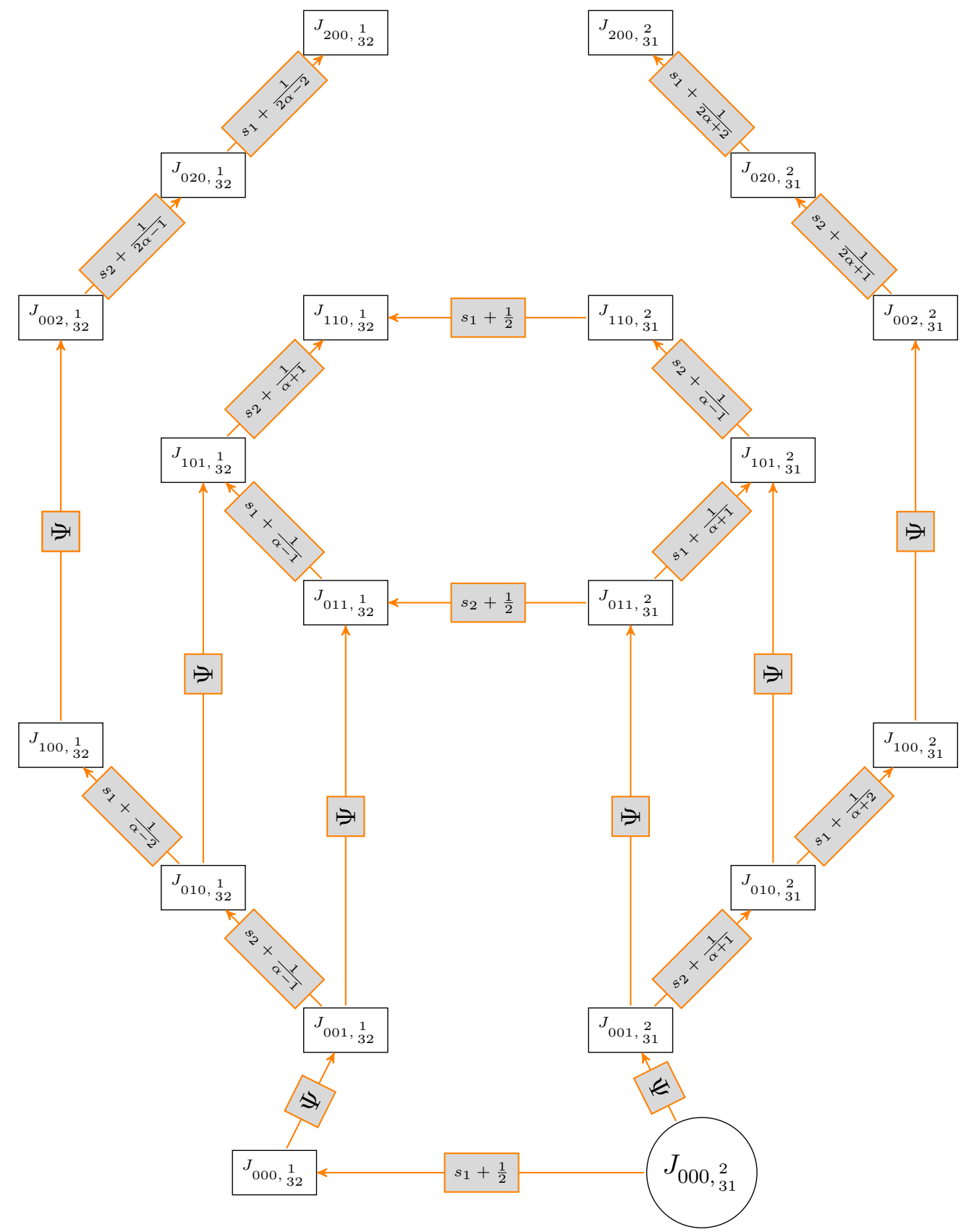

Figure 2. First values of the polynomials $J_{v, \tau}$ for $\lambda=21\left(s_{i}\right.$ means $\left.s_{i} \otimes s_{i}\right)$.

Example 4.5. Consider sign representation associated to the partition $\left[1^{N}\right]$. The set $\operatorname{Tab}_{\left[1^{N}\right]}$ 1

contains a unique element $\tau=\underset{N}{\vdots}$. Hence, we can omit $\tau$ when we write the polynomials of $M_{\left[1^{N}\right]}$. One can see that the corresponding Jack polynomials are equal to the standard ones for the coefficient $-\alpha$. Indeed, since $\tau s_{i j}=-\tau$ one has

$$
P \mathfrak{D}_{i} \simeq(P \otimes \tau) \mathfrak{D}_{i}=(P \otimes \tau)\left(\frac{\partial}{\partial x_{i}}+\frac{1}{\alpha} \sum_{i \neq j} \partial_{i j} \otimes s_{i, j}\right)=(P \otimes \tau)\left(\frac{\partial}{\partial x_{i}}-\frac{1}{\alpha} \sum_{i \neq j} \partial_{i j} \otimes 1\right)
$$




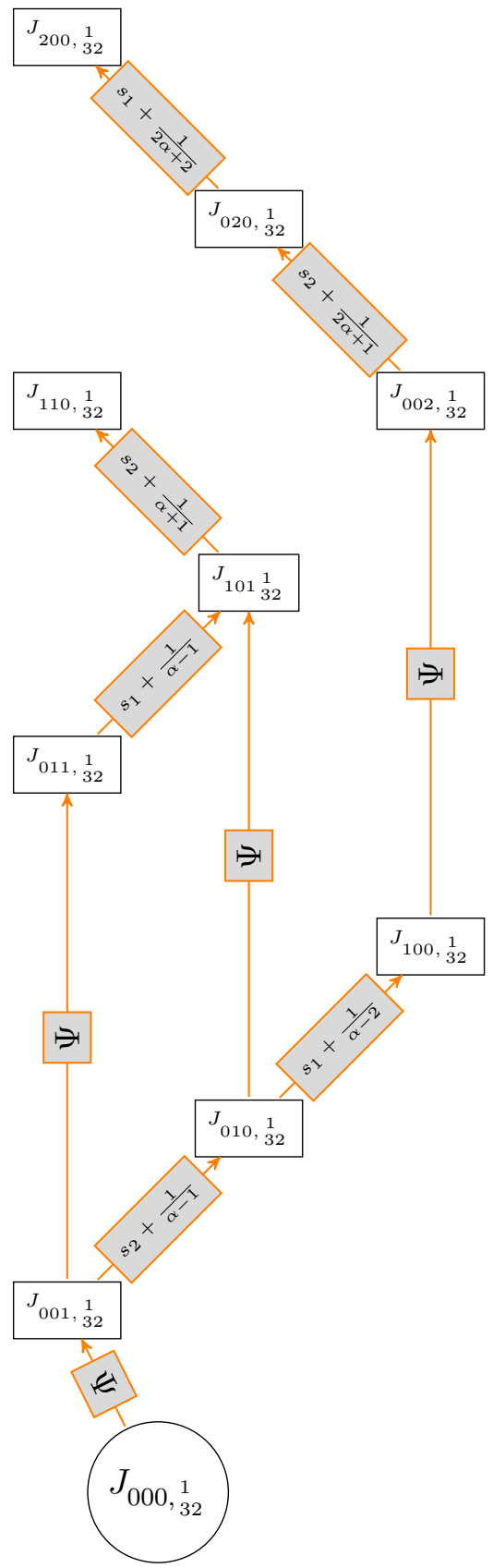

Figure 3. First values of the polynomials $J_{v,{ }_{32}}$.

Hence, the Cherednik-Dunkl operator $\mathfrak{U}_{i}=x_{i} \mathfrak{D}_{i}-\frac{1}{\alpha} \sum_{j=1}^{i-1} s_{i j} \otimes s_{i j}$ acts on $M_{\left[1^{N}\right]}$ as the operator $\mathfrak{U}_{i}$ acts on $M_{[N]}$ but for the parameter $-\alpha$.

Example 4.6. Let us explain the method on a bigger example: $J_{[0,0,2,1,1,0], \tau}$, for $\tau:=\begin{aligned} & 43 \\ & 6521\end{aligned}$. First, we obtain the vector $[0,0,2,1,1,0]$ from $[0,0,0,0,0,0]$ by the following sequence of operations:

$$
\begin{aligned}
& {[0,0,0,0,0,0] \stackrel{\Psi}{\rightarrow}[0,0,0,0,0,1] \stackrel{s_{5}}{\rightarrow}[0,0,0,0,1,0] \stackrel{s_{4}}{\rightarrow}[0,0,0,1,0,0] \stackrel{s_{3}}{\rightarrow}[0,0,1,0,0,0]} \\
& \quad \stackrel{s_{2}}{\rightarrow}[0,1,0,0,0,0] \stackrel{s_{1}}{\rightarrow}[1,0,0,0,0,0] \stackrel{\Psi}{\rightarrow}[0,0,0,0,0,2] \stackrel{s_{5}}{\rightarrow}[0,0,0,0,2,0] \stackrel{\Psi}{\rightarrow}[0,0,0,2,0,1]
\end{aligned}
$$




$$
\stackrel{s 5}{\rightarrow}[0,0,0,2,1,0] \stackrel{\Psi}{\rightarrow}[0,0,2,1,0,1] \stackrel{s 5}{\rightarrow}[0,0,2,1,1,0] .
$$

Replace $\Psi$ by $\Psi^{\alpha}$ in the list of the operations, the associated sequence is

$$
\begin{aligned}
& \zeta_{[0,0,0,0,0,0]}=[3,2,0,-1,1,0] \stackrel{\Psi^{\alpha}}{\rightarrow} \zeta_{[0,0,0,0,0,1]}=[2,0,-1,1,0, \alpha+3] \\
& \quad \stackrel{s_{5}}{\rightarrow} \zeta_{[0,0,0,0,1,0]}=[2,0,-1,1, \alpha+3,0] \stackrel{s_{4}}{\rightarrow} \zeta_{[0,0,0,1,0,0]}=[2,0,-1, \alpha+3,1,0] \\
& \quad \stackrel{s_{3}}{\rightarrow} \zeta_{[0,0,1,0,0,0]}=[2,0, \alpha+3,-1,1,0] \stackrel{s_{2}}{\rightarrow} \zeta_{[0,1,0,0,0,0]}=[2, \alpha+3,0,-1,1,0] \\
& \quad \stackrel{s_{3}}{\rightarrow} \zeta_{[1,0,0,0,0,0]}=[\alpha+3,2,0,-1,1,0] \stackrel{\Psi^{\alpha}}{\rightarrow} \zeta_{[0,0,0,0,0,2]}=[2,0,-1,1,0,2 \alpha+3] \\
& \quad \stackrel{s_{5}}{\rightarrow} \zeta_{[0,0,0,0,2,0]}=[2,0,-1,1,2 \alpha+3,0] \stackrel{\Psi^{\alpha}}{\rightarrow} \zeta_{[0,0,0,2,0,1]}=[0,-1,1,2 \alpha+3,0, \alpha+2] \\
& \quad \stackrel{s_{5}}{\rightarrow} \zeta_{[0,0,0,2,1,0]}=[0,-1,1,2 \alpha+3, \alpha+2,0] \stackrel{\Psi^{\alpha}}{\rightarrow} \zeta_{[0,0,2,1,0,1]}=[-1,1,2 \alpha+3, \alpha+2,0, \alpha] \\
& \quad \stackrel{s_{5}}{\rightarrow} \zeta_{[0,0,2,1,1,0]}=[-1,1,2 \alpha+3, \alpha+2, \alpha, 0] .
\end{aligned}
$$

Now, to obtain the vector-valued Jack polynomial, it suffices to start from $1 \otimes \begin{array}{llll}4 & 3 & \\ 6 & 5 & 2 & 1\end{array}$ and act successively with the affine operator $\Psi$ (when reading $\Psi^{\alpha}$ ) and with $s_{i} \otimes s_{i}+\frac{1}{\zeta[i+1]-\zeta[i]}$ (when reading $s_{i}$ ).

In conclusion, the computation of vector-valued Jack for a given RST is completely independent of the computations of the vector-valued Jack indexed by the other RST with the same shape.

\subsection{Normalization}

The space $V_{\lambda}$ spanned by the RST $\tau$ of the same shape $\lambda$ is naturally endowed (up to a multiplicative constant) by $\mathfrak{S}_{N}$-invariant scalar product $\langle,\rangle_{0}$ with respect to which the $\mathrm{RST}$ are pairwise orthogonal. As in [4], we set

$$
\|\tau\|^{2}=\prod_{\substack{1 \leq i<j \leq N \\ \mathrm{CT}_{\tau}\left[\bar{i}<<\mathrm{CT}_{\tau}[j]-1\right.}} \frac{\left(\mathrm{CT}_{\tau}[i]-\mathrm{CT}_{\tau}[j]-1\right)\left(\mathrm{CT}_{\tau}[i]-\mathrm{CT}_{\tau}[j]+1\right)}{\left(\mathrm{CT}_{\tau}[i]-\mathrm{CT}_{\tau}[j]\right)^{2}}
$$

As in [4], we consider the contravariant form $\langle$,$\rangle on the space M_{\lambda}$ which is the symmetric $\mathfrak{S}_{N}$-invariant form extending $\langle,\rangle_{0}$ and such that the Dunkl operator $\mathfrak{D}_{i}$ is the adjoint to the multiplication by $x_{i}$ (see appendix in [4] for more details).

The operator $x_{i} \mathfrak{D}_{i}$ is self adjoint and the adjoint of $\sigma \in \mathfrak{S}_{N}$ is $\sigma^{-1}$. Since $s_{i j}=s_{i j}^{-1}$ is self adjoint, $\mathfrak{U}_{i}$ is self-adjoint for the form $\langle$,$\rangle and the polynomials J_{v, \tau}$ are pairwise orthogonal.

Let us compute their squared norms $\left\|J_{v, \tau}\right\|^{2}$ (the bilinear form is nonsingular for generic $\alpha$ and positive definite for $\alpha$ in some subset of $\mathbb{R}[6]$ ). The method is essentially the same as in [4] and we show that the result can be read in the Yang-Baxter graph. More precisely, one has

\section{Proposition 4.7.}

1. $\left\|J_{(v, \tau) s_{i}}\right\|^{2}=\frac{\left(\zeta_{v, \tau}[i+1]-\zeta_{v, \tau}[i]-1\right)\left(\zeta_{v, \tau}[i+1]-\zeta_{v, \tau}[i]+1\right)}{\left(\zeta_{v, \tau}[i+1]-\zeta_{v, \tau}[i]\right)^{2}}\left\|J_{v, \tau}\right\|^{2}$.

2. $\left\|J_{(v, \tau) \Psi}\right\|^{2}=\left(\frac{1}{\alpha} \zeta_{v, \tau}[1]+1\right)\left\|J_{v, \tau}\right\|^{2}$.

Proof. 1. Since

$$
J_{(v, \tau) s_{i}}=J_{v, \tau}\left(s_{i} \otimes s_{i}+\frac{1}{\zeta_{v, \tau}[i+1]-\zeta_{v, \tau}[i]}\right),
$$




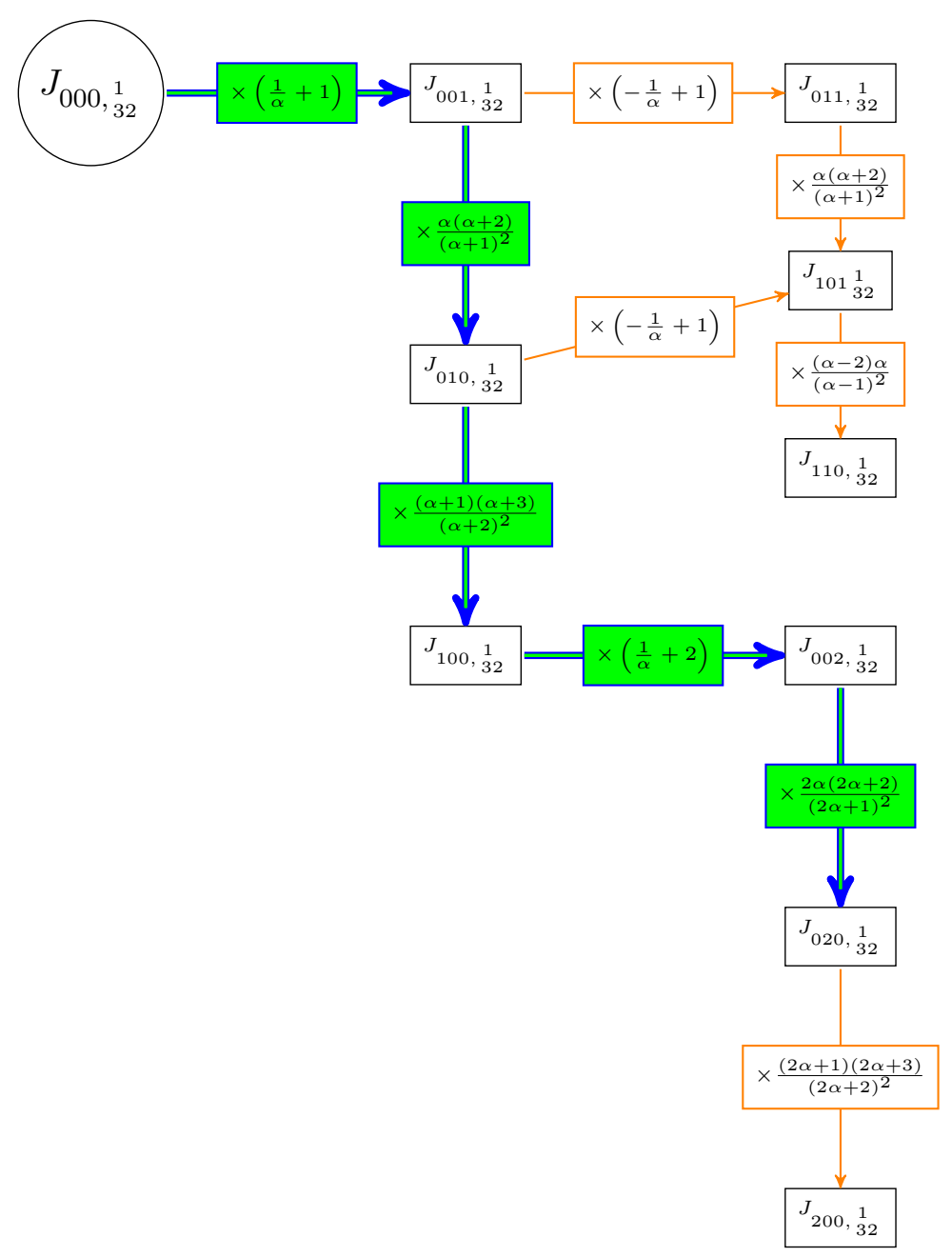

Figure 4. Computation of $\left\|J_{020,1}{ }_{32}\right\|^{2}$ using the graph $G_{32}$.

one obtains

$$
\left\|J_{v, \tau}\left(s_{i} \otimes s_{i}\right)\right\|^{2}=\left\|J_{(v, \tau) s_{i}}\right\|^{2}+\frac{1}{\left(\zeta_{v, \tau}[i+1]-\zeta_{v, \tau}[i]\right)^{2}}\left\|J_{v, \tau}\right\|^{2} .
$$

But $\left\|J_{v, \tau}\left(s_{i} \otimes s_{i}\right)\right\|^{2}=\left\|J_{v, \tau}\right\|^{2}$, which gives the result.

2. One has

$$
\begin{aligned}
\left\|J_{(v, \tau) \Psi}\right\|^{2} & =\left\|J_{v, \tau}(\theta \otimes \theta) x_{N}\right\|^{2}=\left\langle J_{v, \tau}(\theta \otimes \theta), J_{v, \tau}(\theta \otimes \theta) x_{N} \mathfrak{D}_{N}\right\rangle \\
& =\left\langle J_{v, \tau}(\theta \otimes \theta), J_{v, \tau} x_{1} \mathfrak{D}_{1}(\theta \otimes \theta)\right\rangle=\left\langle J_{v, \tau}, J_{v, \tau} x_{1} \mathfrak{D}_{1}\right\rangle ;
\end{aligned}
$$

recall that $\theta=s_{1} s_{2} \cdots s_{N-1}$. Since $\mathfrak{U}_{1}=x_{1} \mathfrak{D}_{1}$, one obtains the results.

Example 4.8. Let again $\tau=\begin{aligned} & 2 \\ & 3\end{aligned} \quad 1$, we compute the normalization following the Yang-Baxter graph (see Fig. 4).

For instance:

$$
\begin{aligned}
\left\|J_{[020], \tau}\right\|^{2} & =\|\tau\|^{2}\left(1+\frac{1}{\alpha}\right)\left(\frac{\alpha(\alpha+2)}{(\alpha+1)^{2}}\right)\left(\frac{(\alpha+1)(\alpha+3)}{(\alpha+2)^{2}}\right)\left(2+\frac{1}{\alpha}\right)\left(\frac{2 \alpha(2 \alpha+2)}{(2 \alpha+1)^{2}}\right) \\
& =\frac{4(\alpha+3)(\alpha+1)}{(2 \alpha+1)(\alpha+2)} .
\end{aligned}
$$




\section{Symmetrization and antisymmetrization}

In [2], Baker and Forrester investigated the coefficients and the norm of the symmetric Jack polynomials by symmetrizing the nonsymmetric Jack polynomials. The symmetrization method was used in [5] for the polynomials associated with the complex groups $G(r, p, N)$. In this section, we generalize their results and obtain symmetric and antisymmetric vector-valued Jack polynomials.

\subsection{Non-affine connectivity}

Let us denote by $H_{\lambda}$ the graph obtained from $G_{\lambda}$ by removing the affine edges, all the falls and the vertex $\varnothing$. The purpose of this section is to investigate the connected components of $H_{\lambda}$. Recall that $v^{+}$is the unique decreasing partition obtained by permuting the entries of $v$.

Definition 5.1. Let $v \in \mathbb{N}^{N}$ and $\tau \in \operatorname{Tab}_{\lambda}$ ( $\lambda$ partition). We define the filling $T(\tau, v)$ obtained by replacing $i$ by $v^{+}[i]$ in $\tau$ for each $i$.

Proposition 5.2. Two 4-tuples $(\tau, \zeta, v, \sigma)$ and $\left(\tau^{\prime}, \zeta^{\prime}, v^{\prime}, \sigma^{\prime}\right)$ are in the same connected component of $H_{\lambda}$ if and only if $T(\tau, v)=T\left(\tau^{\prime}, v^{\prime}\right)$.

Proof. Remark first that the steps and correct jumps preserve $T(\tau, v)$. Indeed steps leave invariant the pairs $\left(\tau, v^{+}\right)$whilst the correct jumps act on the RST by $\tau s_{\sigma_{v}[i]}$ where $v[i]=v[i+1]$ (or equivalently by $\tau s_{j}$ where $v^{+}[j]=v^{+}[j+1]$. Hence, we show that if $(\tau, \zeta, v, \sigma)$ is connected to $\left(\tau^{\prime}, \zeta^{\prime}, v^{\prime}, \sigma^{\prime}\right)$ then $T(\tau, v)=T\left(\tau^{\prime}, v^{\prime}\right)$.

Let us prove the converse. Suppose that $T(\tau, v)=T\left(\tau^{\prime}, v^{\prime}\right)$. Since $(\tau, \zeta, v, \sigma)\left(\operatorname{resp} . \quad\left(\tau^{\prime}, \zeta^{\prime}\right.\right.$, $\left.\left.v^{\prime}, \sigma^{\prime}\right)\right)$ is connected to $\left(\tau, \zeta, v^{+}, \mathrm{Id}\right)\left(\right.$ resp. $\left.\left(\tau^{\prime}, \zeta^{\prime}, v^{\prime+}, I d\right)\right)$ by steps, it suffices to prove the result for $T(\tau, \mu)=T\left(\tau^{\prime}, \mu\right)$ when $\mu$ is a (decreasing) partition. Let $\rho \in \mathfrak{S}_{N}$ such that $\tau^{\rho}$ (the tableau $\tau$ where the entries have been permuted by $\rho$ ) equals $\tau_{\lambda}$. By construction $T(\tau, \mu)=T\left(\tau_{\lambda}, \mu \rho^{-1}\right)$ and then it suffices to show the result for $T\left(\tau_{\lambda}, \mu \rho^{-1}\right)=T\left(\tau^{\prime \rho}, \mu \rho^{-1}\right)$. Again the connectivity by steps implies that it suffices to prove the result for $T\left(\tau_{\lambda}, \mu\right)=T\left(\tau^{\prime}, \mu\right)$ when $\mu$ is a partition. We will show that if $T\left(\tau_{\lambda}, \mu\right)=T(\tau, \mu)$ then there exists a series of correct jumps from $\left(\tau, \zeta^{\prime}, \mu, \operatorname{Id}\right)$ to $\left(\tau_{\lambda}, \zeta, \mu, \mathrm{Id}\right)$ when $\mu$ is a partition. We prove the result by induction on the length of the shortest permutation $\omega$ such that $\tau \omega=\tau_{\lambda}$ for the weak order. The base point of the induction is straightforward. Now, choose $i$ such that $\omega[i]>\omega[i+1]$ and $i$ and $i+1$ are neither in the same row nor in the same column in $\tau$ then $\omega=s_{i} \omega^{\prime}$ where $\ell\left(\omega^{\prime}\right)<\ell(\omega)$. Since $T(\tau, \mu)=$ $T\left(\tau^{\prime}, \mu\right)$, this means that $\mu[i]=\mu[i+1]$ and hence, there is a correct jump from $\left(\tau, \zeta^{\prime}, \mu, \operatorname{Id}\right)$ to $\left(\tau^{(i, i+1)}, \zeta^{\prime} s_{i}, \mu, \mathrm{Id}\right)$. By the induction hypothesis, this shows the result.

This shows that the connected components of $H_{\lambda}$ are indexed by the $T(\tau, \mu)$ where $\mu$ is a partition.

Definition 5.3. We will denote by $H_{T}$ the connected component associated to $T$ in $H_{\lambda}$. The component $H_{T}$ will be said to be 1-compatible if $T$ is a column-strict tableau. The component $H_{T}$ will be said to be (-1)-compatible if $T$ is a row-strict tableau.

Example 5.4. Let $\mu=[2,1,1,0,0]$ and $\lambda=[3,2]$. There are four connected components with vertices labeled by permutations of $\mu$ in $H_{\lambda}$ (see Fig. 5). The possible values of $T(\tau, \mu)$ are

$$
\begin{aligned}
& \begin{array}{llll}
12 & 02 & 01 &
\end{array} \\
& \text { 001'011'012 and 002' }
\end{aligned}
$$

squared in red in Fig. 5. The 1-compatible components are $H_{12}$ and $H_{11}$ while there is only one $(-1)$-compatible component $H_{012}$. The component $H_{011}$ is neither 1-compatible nor $(-1)$ compatible.

The component $H_{12}$ contains vertices of $G_{542}$ and $G_{543}$ connected by jumps. 

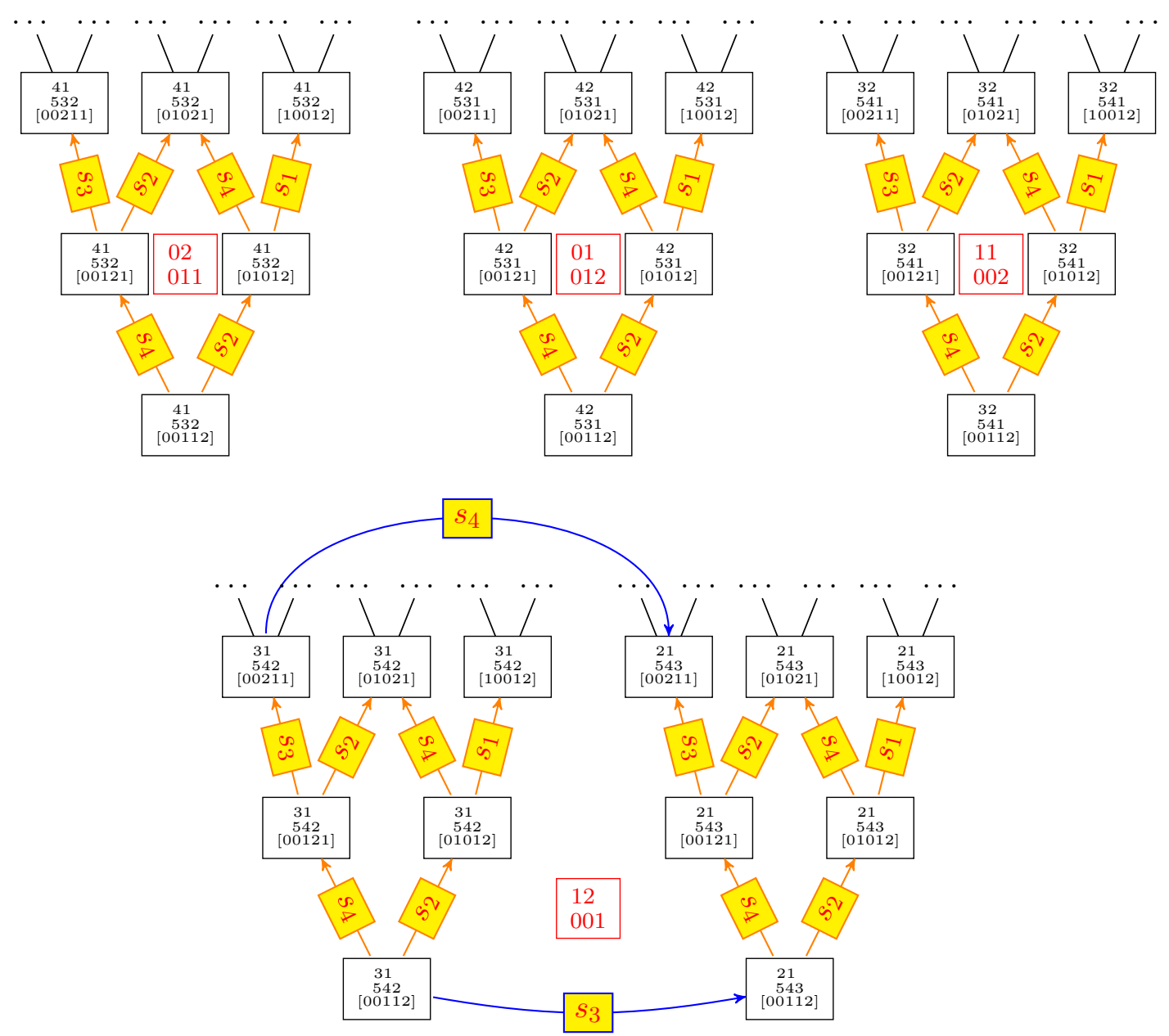

Figure 5. Some connected components of $H_{32}$.

We we use the following result in the sequel, its proof is easy and left to the reader.

Proposition 5.5. Let $(\tau, \zeta, v, \sigma)$ be a vertex of $H_{T}$ such that $(\tau, \zeta, v, \sigma) s_{i}=\varnothing$. One has

1. If $H_{T}$ is 1-compatible then $\sigma[i]$ and $\sigma[i+1](=\sigma[i]+1)$ are in the same row in $\tau$.

2. If $H_{T}$ is $(-1)$-compatible then $\sigma[i]$ and $\sigma[i+1](=\sigma[i]+1)$ are in the same column in $\tau$.

The following definition is used to find a RST corresponding to a filling of a shape.

Definition 5.6. Let $T$ be a filling of shape $\lambda$, the standardization $\operatorname{std}(T)$ of $T$ is the reverse standard tableau with shape $\lambda$ obtained by the following process:

1. Denote by $|T|_{i}$ the number of occurrences of $i$ in $T$

2. Read the tableau $T$ from the left to the right and the bottom to the top and replace successively each occurrence of $i$ by the numbers $N-|T|_{0}-\cdots-|T|_{i-1}, N-|T|_{0}-\cdots-$ $|T|_{i-1}-1, \ldots, N-|T|_{0}-\cdots-|T|_{i}$.

Alternatively, one has

$$
\begin{aligned}
\operatorname{std}(T)[i, j]:= & \#\{(k, l): T[k, l]>T[i, j]\}+\#\{(k, l): l>j, T[k, l]=T[i, j]\} \\
& +\#\{(k, j): k \geq i, T[k, j]=T[i, j]\} .
\end{aligned}
$$

We will denote by $\lambda_{T}$ the unique partition obtained by sorting in the decreasing order all the entries of $T$. 
Example 5.7. Pictorially, reading ${ }_{002}^{01}$ one obtains

\begin{tabular}{ccc|cc}
0 & 0 & 2 & 0 & 1 \\
\hline 0 & 0 &. & 0 &. \\
. &. &. &. & 1 \\
. &. & 2 &. &.
\end{tabular}

Renumbering in increasing order from the bottom to the top and the right to the left, one reads

\begin{tabular}{ccc|cc}
0 & 0 & 2 & 0 & 1 \\
\hline 5 & 4 &. & 3 &. \\
. &. &. &. & 2 \\
. &. & 1 &. &.
\end{tabular}

Hence, we have $\operatorname{std}\left(\begin{array}{l}01 \\ 002\end{array}\right)={ }_{541}^{32}$ and $\lambda_{002}=[21000]$.

Note that each $H_{T}$ has a unique sink (that is a vertex with no outward edge) and this vertex is labeled by $\left(\operatorname{std}(T), \zeta_{T}, \lambda_{T}, I d\right)$ for a certain vector $\zeta_{T}$ and a unique root.

Example 5.8. Consider the tableau $T={ }_{00}^{01}$. Its standardization is $\operatorname{std}(T)={ }_{43}^{21}$ and the graph $H_{T}$ is:

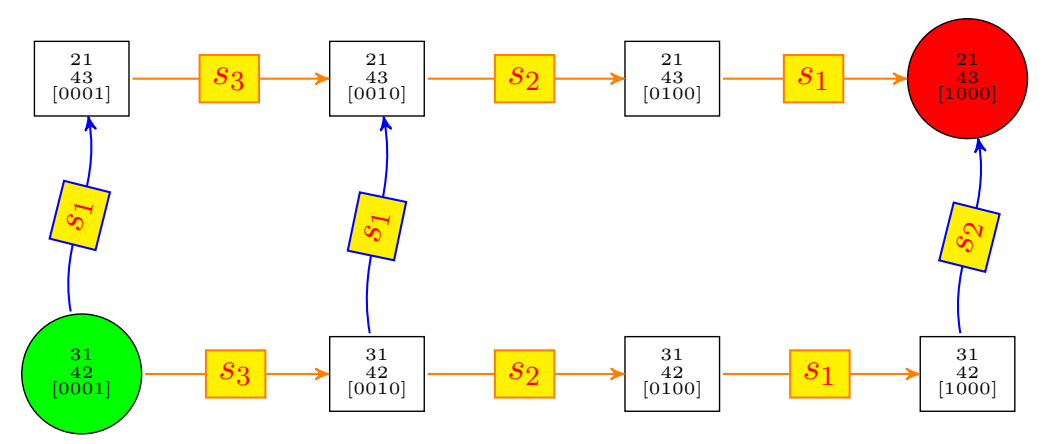

The sink is denoted by a red disk and the root by a green disk.

\subsection{Symmetric and antisymmetric Jack polynomials}

For convenience, let us define:

$$
(v, \tau) s_{i}=\left(v^{\prime}, \tau^{\prime}\right) \quad \text { if } \quad(\tau, \zeta, v, \sigma) s_{i}=\left(\tau^{\prime}, \zeta^{\prime}, v^{\prime}, \sigma^{\prime}\right)
$$

and

$$
(v, \tau) s_{i}=\varnothing \quad \text { if } \quad(\tau, \zeta, v, \sigma) s_{i}=\varnothing .
$$

Denote also, $J_{\varnothing}:=0$.

Let $(\tau, \zeta, v, \sigma)$ be a vertex of $H_{T}$, set $b_{v, \tau}[i]=\frac{1}{\zeta_{v, \tau}[i+1]-\zeta_{v, \tau}[i]}$ and $c_{v, \tau}[i]=\frac{\zeta_{v, \tau}[i]-\zeta_{v, \tau}[i+1]}{\zeta_{v, \tau}[i]-\zeta_{v, \tau}[i+1]+1}$.

Note that

$$
1+c_{v, \tau}[i] b_{v, \tau}[i]=c_{v, \tau}[i]
$$

and

$$
c_{v, \tau}[i]\left(1-b_{v, \tau}[i]^{2}\right)-b_{v, \tau}[i]=1 .
$$

Let $H_{T}$ be a 1-compatible component of $G_{\lambda}$. For each vertex $(\tau, \zeta, v, \sigma)$ of $H_{T}$, we define the coefficient $\mathfrak{E}_{v, \tau}$ by the following induction: 
1. $\mathfrak{E}_{v, \tau}=1$ if there is no arrows of the form

$$
\left(\tau^{\prime}, \zeta^{\prime}, v^{\prime}, \sigma^{\prime}\right)-s_{i} \longrightarrow(\tau, \zeta, v, \sigma)
$$

in $H_{T}$.

2. $\mathfrak{E}_{v, \tau}=\frac{\zeta^{\prime}[i+1]-\zeta^{\prime}[i]}{\zeta^{\prime}[i+1]-\zeta^{\prime}[i]-1} \mathfrak{E}_{v^{\prime}, \tau^{\prime}}=\frac{\zeta[i+1]-\zeta[i]}{\zeta[i+1]-\zeta[i]+1} \mathfrak{E}_{v^{\prime}, \tau^{\prime}}=c_{v^{\prime}, \tau^{\prime}} \mathfrak{E}_{v^{\prime}, \tau^{\prime}}$ if there is an arrow

$$
\left(\tau^{\prime}, \zeta^{\prime}, v^{\prime}, \sigma^{\prime}\right) \longrightarrow s_{i} \longrightarrow(\tau, \zeta, v, \sigma)
$$

in $H_{T}$.

The symmetric group acts on the spectral vectors $\zeta$ by permuting their components. Hence the value of $\mathfrak{E}_{v, \tau}$ does not depend on the path used for its computation and the $\mathfrak{E}_{v, \tau}$ are well defined. Indeed, it suffices to check that the definition is compatible with the commutations $s_{i} s_{j}=s_{j} s_{i}$ with $|i-j|>1$ and the braid relations $s_{i} s_{i+1} s_{i}=s_{i+1} s_{i} s_{i+1}$.

Let us first prove the compatibility with the commutation relations. Suppose

$$
\left(\tau_{0}, \zeta_{0}, v_{0}, \sigma_{0}\right) s_{i} s_{j}=\left(\tau_{1}, \zeta_{1}, v_{1}, \sigma_{1}\right) s_{j}=\left(\tau_{2}, \zeta_{2}, v_{2}, \sigma_{2}\right)
$$

with $|i-j|>1$ and

$$
\left(\tau_{0}, \zeta_{0}, v_{0}, \sigma_{0}\right) s_{j} s_{i}=\left(\tau_{1}^{\prime}, \zeta_{1}^{\prime}, v_{1}^{\prime}, \sigma_{1}^{\prime}\right) s_{i}=\left(\tau_{2}^{\prime}, \zeta_{2}^{\prime}, v_{2}^{\prime}, \sigma_{2}^{\prime}\right)
$$

Note that $\tau_{2}^{\prime}=\tau_{2}, \zeta_{2}^{\prime}=\zeta_{2}, v_{2}^{\prime}=v_{2}$ and $\sigma_{2}^{\prime}=\sigma_{2}$. But, since the symmetric group acts on $\zeta$ by permuting its components, one has

$$
\zeta_{2}[j+1]=\zeta_{1}^{\prime}[j+1], \quad \zeta_{2}[j]=\zeta_{1}^{\prime}[j], \quad \zeta_{1}[j+1]=\zeta_{2}^{\prime}[j+1] \quad \text { and } \quad \zeta_{1}[j]=\zeta_{2}^{\prime}[j] .
$$

Hence,

$$
\begin{aligned}
\frac{\zeta_{2}[j+1]-\zeta_{2}[j]}{\zeta_{2}[j+1]-\zeta_{2}[j]+1} \cdot \frac{\zeta_{1}[i+1]-\zeta_{1}[i]}{\zeta_{1}[i+1]-\zeta_{1}[i]+1} & =\frac{\zeta_{1}[i+1]-\zeta_{1}[i]}{\zeta_{1}[i+1]-\zeta_{1}[i]+1} \cdot \frac{\zeta_{2}[j+1]-\zeta_{2}[j]}{\zeta_{2}[j+1]-\zeta_{2}[j]+1} \\
& =\frac{\zeta_{1}^{\prime}[i+1]-\zeta_{1}^{\prime}[i]}{\zeta_{1}^{\prime}[i+1]-\zeta_{1}^{\prime}[i]+1} \cdot \frac{\zeta_{2}^{\prime}[j+1]-\zeta_{2}^{\prime}[j]}{\zeta_{2}^{\prime}[j+1]-\zeta_{2}^{\prime}[j]+1},
\end{aligned}
$$

and the definition of $\mathfrak{E}_{v, \tau}$ is compatible with the commutations.

Now, let us show that the definition is compatible with the braid relations and set

$$
\left(\tau_{0}, \zeta_{0}, v_{0}, \sigma_{0}\right) s_{i} s_{i+1} s_{i}=\left(\tau_{1}, \zeta_{1}, v_{1}, \sigma_{1}\right) s_{i+1} s_{i}=\left(\tau_{2}, \zeta_{2}, v_{2}, \sigma_{2}\right) s_{i}=\left(\tau_{3}, \zeta_{3}, v_{3}, \sigma_{3}\right)
$$

and

$$
\left(\tau_{0}, \zeta_{0}, v_{0}, \sigma_{0}\right) s_{i+1} s_{i} s_{i+1}=\left(\tau_{1}^{\prime}, \zeta_{1}^{\prime}, v_{1}^{\prime}, \sigma_{1}^{\prime}\right) s_{i} s_{i+1}=\left(\tau_{2}^{\prime}, \zeta_{2}^{\prime}, v_{2}^{\prime}, \sigma_{2}^{\prime}\right) s_{i+1}=\left(\tau_{3}^{\prime}, \zeta_{3}^{\prime}, v_{3}^{\prime}, \sigma_{3}^{\prime}\right)
$$

Note that $\tau_{3}^{\prime}=\tau_{3}, \zeta_{3}^{\prime}=\zeta_{3}, v_{3}^{\prime}=v_{3}$ and $\sigma_{3}^{\prime}=\sigma_{3}$. Since the symmetric group acts on $\zeta$ by permuting its components, one has

$$
\begin{aligned}
\zeta_{3}[i+1] & =\zeta_{1}^{\prime}[i+1], \quad \zeta_{3}[i]=\zeta_{1}^{\prime}[i+1], \\
\zeta_{2}[i+2] & =\zeta_{2}^{\prime}[i+1], \quad \zeta_{2}[i+1]=\zeta_{2}^{\prime}[i], \\
\zeta_{1}[i+1] & =\zeta_{2}^{\prime}[i+2] \quad \text { and } \quad \zeta_{1}[i]=\zeta_{3}^{\prime}[i+1] .
\end{aligned}
$$


Hence,

$$
\begin{aligned}
& \frac{\zeta_{3}[i+1]-\zeta_{3}[i]}{\zeta_{3}[i+1]-\zeta_{3}[i]+1} \cdot \frac{\zeta_{2}[i+2]-\zeta_{2}[i+1]}{\zeta_{2}[i+2]-\zeta_{2}[i+1]+1} \cdot \frac{\zeta_{1}[i+1]-\zeta_{1}[i]}{\zeta_{1}[i+1]-\zeta_{1}[i]+1} \\
& \quad=\frac{\zeta_{1}[i+1]-\zeta_{1}[i]}{\zeta_{1}[i+1]-\zeta_{1}[i]+1} \cdot \frac{\zeta_{2}[i+2]-\zeta_{2}[i+1]}{\zeta_{2}[i+2]-\zeta_{2}[i+1]+1} \cdot \frac{\zeta_{3}[i+1]-\zeta_{3}[i]}{\zeta_{3}[i+1]-\zeta_{3}[i]+1} \\
& \quad=\frac{\zeta_{3}^{\prime}[i+2]-\zeta_{3}^{\prime}[i+1]}{\zeta_{3}^{\prime}[i+2]-\zeta_{3}^{\prime}[i+1]+1} \cdot \frac{\zeta_{2}^{\prime}[i+1]-\zeta_{2}^{\prime}[i]}{\zeta_{2}^{\prime}[i+1]-\zeta_{2}^{\prime}[i]+1} \cdot \frac{\zeta_{1}^{\prime}[i+2]-\zeta_{1}^{\prime}[i+1]}{\zeta_{1}^{\prime}[i+2]-\zeta_{1}^{\prime}[i+1]+1}
\end{aligned}
$$

and the definition is compatible with the braid relations.

Define the symmetrization operator

$$
\mathfrak{S}:=\sum_{\omega \in \mathfrak{S}_{N}} \omega \otimes \omega
$$

We will say that a polynomial is symmetric if it is invariant under the action of $s_{i} \otimes s_{i}$ for each $i<N$.

\section{Theorem 5.9.}

1. Let $H_{T}$ be a connected component of $G_{\lambda}$. For each vertex $(\tau, \zeta, v, \sigma)$ of $H_{T}$, the polynomial $J_{v, \tau} \mathfrak{S}$ equals $J_{\lambda_{T}, \operatorname{std}(T)} \mathfrak{S}$ up to a multiplicative constant.

2. One has $J_{\lambda_{T}, \operatorname{std}(T)} \mathfrak{S} \neq 0$ if and only if $H_{T}$ is 1-compatible.

3. More precisely, when $H_{T}$ is 1-compatible, the polynomial

$$
J_{T}=\sum_{(\tau, \zeta, v, \sigma)} \mathfrak{E}_{v, \tau} J_{v, \tau}
$$

is symmetric.

Proof. 1. Let us prove the first assertion by induction on the length of a path from $(\tau, \zeta, v, \sigma)$ to $\left(\operatorname{std}(T), \zeta_{T}, \lambda_{T}, \sigma\right)$ in $H_{T}$. Let $\left(\tau^{\prime}, \zeta^{\prime}, v^{\prime}, \sigma^{\prime}\right)$ such that

$$
(\tau, \zeta, v, \sigma)-s_{i} \rightarrow\left(\tau^{\prime}, \zeta^{\prime}, v^{\prime}, \sigma^{\prime}\right)
$$

is not a jump in $H_{T}$ (hence, $-1<b_{v, \tau}[i]<1$ ). It follows that

$$
J_{v, \tau} \mathfrak{S}=\frac{1}{1-b_{v, \tau}[i]^{2}}\left(J_{v^{\prime}, \tau^{\prime}}\left(s_{i} \otimes s_{i}\right)+b_{v, \tau}[i] J_{v^{\prime}, \tau^{\prime}}\right) \mathfrak{S}=\frac{1}{1-b_{v, \tau}[i]^{2}}\left(1+b_{v, \tau}[i]\right) J_{v^{\prime}, \tau^{\prime}} \mathfrak{S}
$$

By induction $J_{v^{\prime}, \tau^{\prime}} \mathfrak{S}$ is proportional to $J_{\lambda_{T}, \operatorname{std}(T)}$, which ends the proof.

2. If $H_{T}$ is not 1-compatible, then there exists $s_{i}$ such that $J_{\lambda_{T}, \operatorname{std}(T)}\left(s_{i} \otimes s_{i}\right)=-J_{\lambda_{T}, \operatorname{std}(T)}$. Hence, since $\mathfrak{S}=\left(s_{i} \otimes s_{i}\right) \mathfrak{S}$, one obtains $J_{\lambda_{T}, \operatorname{std}(T)} \mathfrak{S}=0$.

3. Let us prove that, when $H_{T}$ is 1-compatible, $J_{T}\left(s_{i} \otimes s_{i}\right)=J_{T}$ for any $i$. Fix $i$ and decompose $J_{T}:=J^{+}+J_{0}+J^{-}$where

$$
J^{+}=\sum_{\tau, v}^{+} \mathfrak{E}_{\tau, v} J_{v, \tau}
$$

where $\sum^{+}$means that the sum is over the pairs $(\tau, v)$ such that there exists an arrow

$$
\left(\tau^{\prime}, \zeta^{\prime}, v^{\prime}, \sigma^{\prime}\right)-s_{i} \longrightarrow(\tau, \zeta, v, \sigma)
$$


in $H_{T}$,

$$
J^{-}=\sum_{\tau, v}^{-} \mathfrak{E}_{\tau, v} J_{v, \tau}
$$

where $\sum^{-}$means that the sum is over the pairs $(\tau, v)$ such that there exists an arrow

$$
(\tau, \zeta, v, \sigma)-s_{i} \longrightarrow\left(\tau^{\prime}, \zeta^{\prime}, v^{\prime}, \sigma^{\prime}\right)
$$

in $H_{T}$ and

$$
J_{0}=\sum^{0} \mathfrak{E}_{\tau, v} J_{v, \tau}
$$

where $\sum^{0}$ means that the sum is over the pairs $(\tau, v)$ such that there exists an arrow

$$
(\tau, \zeta, v, \sigma)-s_{i} \rightarrow \varnothing
$$

in $G_{T}$ (equivalently there is no arrow from $(\tau, \zeta, v, \sigma)$ labeled by $s_{i}$ in $\left.H_{T}\right)$. Suppose that

$$
(\tau, \zeta, v, \sigma)-s_{i} \rightarrow \varnothing
$$

is a fall in $G_{T}$, then

$$
J_{v, \tau}\left(s_{i} \otimes s_{i}\right)=J_{(v, \tau) s_{i}}-b_{v, \tau}[i] J_{v, \tau}=-b_{v, \tau}[i] J_{v, \tau} .
$$

Since, $H_{T}$ is 1-compatible Proposition 5.5 implies that $i$ and $i+1$ are in the same row. Hence, $b_{v, \tau}[i]=-1$ and $J_{v, \tau}\left(s_{i} \otimes s_{i}\right)=J_{v, \tau}$. It follows that $J_{0}\left(s_{i} \otimes s_{i}\right)=J_{0}$.

Now, let

$$
(\tau, \zeta, v, \sigma)-s_{i} \longrightarrow\left(\tau^{\prime}, \zeta^{\prime}, v^{\prime}, \sigma^{\prime}\right)
$$

be an arrow in $H_{T}$, then

$$
\begin{aligned}
& J_{v, \tau}\left(s_{i} \otimes s_{i}\right)=J_{v^{\prime}, \tau^{\prime}}-b_{v, \tau}[i] J_{v, \tau}, \\
& J_{v^{\prime}, \tau^{\prime}}\left(s_{i} \otimes s_{i}\right)=b_{v, \tau}[i] J_{v^{\prime}, \tau^{\prime}}+\left(1-b_{v, \tau}[i]^{2}\right) J_{v, \tau} \quad \text { and } \quad \mathfrak{E}_{v^{\prime}, \tau^{\prime}}=c_{v, \tau} \mathfrak{E}_{v, \tau} .
\end{aligned}
$$

Hence, equalities (5.1) and (5.2) imply

$$
\begin{aligned}
& \left(\mathfrak{E}_{v, \tau} J_{v, \tau}+\mathfrak{E}_{v^{\prime}, \tau^{\prime}} J_{v^{\prime}, \tau^{\prime}}\right)\left(s_{i} \otimes s_{i}\right)=\mathfrak{E}_{v, \tau}\left(J_{v, \tau}+c_{v, \tau}[i] J_{v^{\prime}, \tau^{\prime}}\right)\left(s_{i} \otimes s_{i}\right) \\
& \quad=\mathfrak{E}_{v, \tau}\left(\left(\left(c_{v, \tau}[i]\left(1-b_{v, \tau}[i]^{2}\right)-b_{v, \tau}[i]\right) J_{v, \tau}+\left(1+c_{v, \tau}[i] b_{v, \tau}[i]\right) J_{v^{\prime}, \tau^{\prime}}\right)\right. \\
& \quad=\mathfrak{E}_{v, \tau}\left(J_{v, \tau}+c_{v, \tau}[i] J_{v^{\prime}, \tau^{\prime}}\right)=\left(\mathfrak{E}_{v, \tau} J_{v, \tau}+\mathfrak{E}_{v^{\prime}, \tau^{\prime}} J_{v^{\prime}, \tau^{\prime}}\right) .
\end{aligned}
$$

This proves that $\left(J^{+}+J^{-}\right)\left(s_{i} \otimes s_{i}\right)=J^{+}+J^{-}$. Hence, $J_{T}\left(s_{i} \otimes s_{i}\right)=J_{T}$ for each $i$ and $J_{T}$ is symmetric.

Example 5.10. Consider the graph $H_{11}$

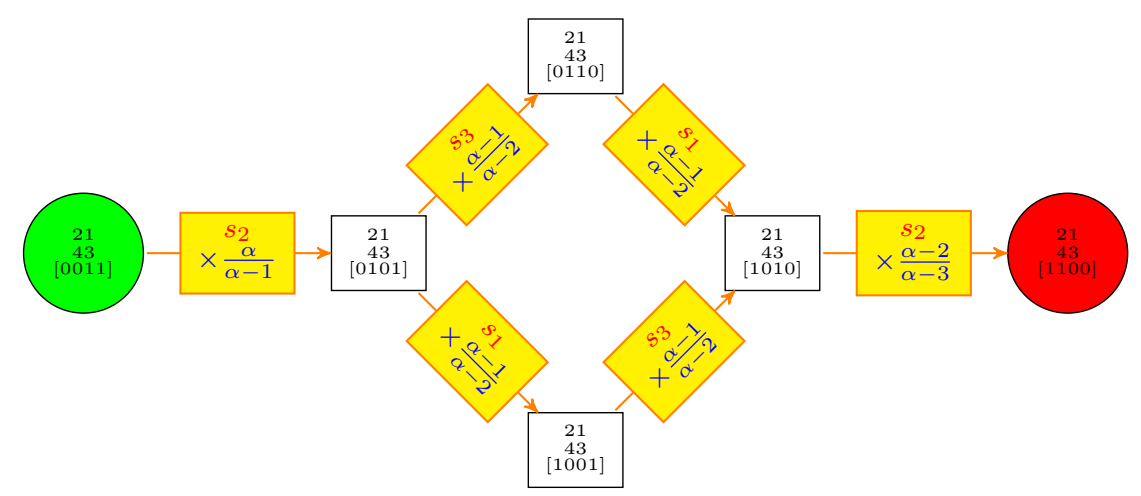


The polynomial

$$
\begin{aligned}
J_{00}= & J_{0011,{ }_{43}^{21}}+\frac{\alpha}{\alpha-1} J_{0101,{ }_{43}^{21}}+\frac{\alpha}{\alpha-2} J_{0110,{ }_{43}^{21}}+\frac{\alpha}{\alpha-2} J_{1001,{ }_{43}^{21}}+\frac{\alpha(\alpha-1)}{(\alpha-2)^{2}} J_{1010,{ }_{43}^{21}} \\
& +\frac{\alpha(\alpha-1)}{(\alpha-2)(\alpha-3)} J_{1100,{ }_{43}^{21}}
\end{aligned}
$$

is symmetric.

Let $H_{T}$ be a connected component, denote by $\operatorname{root}(T)$ the only vertex of $H_{T}$ without inward edge and by $\operatorname{sink}(T)=\left(\operatorname{std}(T), \zeta_{T}, \lambda_{T}, \mathrm{Id}\right)$ the only vertex of $H_{T}$ without outward edge. Denote by $\# H_{T}$ the number of vertices of $H_{T}$. The following proposition allows to compare the polynomial $J_{T}$ to the symmetrization of $J_{\operatorname{root}(T)}$.

Proposition 5.11. One has

$$
J_{T}=\frac{\# H_{T}}{N !} \mathfrak{E}_{\operatorname{sink}(T)} J_{\operatorname{root}(T)} \mathfrak{S} .
$$

Proof. It suffices to compare the coefficient of $J_{\operatorname{sink}(T)}$ in $J_{T}$ and in $J_{\operatorname{root}(T)}$. S. The coefficient of $J_{\operatorname{sink}(T)}$ in $J_{T}$ equals $\mathfrak{E}_{\operatorname{sink}(T)}$ while the coefficient of $J_{\operatorname{sink}(T)}$ in $J_{\operatorname{root}(T)} \mathfrak{S}$ equals $\frac{N !}{\# H}$. Indeed $\frac{N !}{\# H}$ is the order of the stabilizer of $\lambda_{T}$. The leading monomial of $J_{\operatorname{sink}(T)}$ does not appear in any other $J_{v, \tau}$ so its coefficient in the symmetrization of $J_{\operatorname{root}(T)}$ equals the order of the stabilizer.

Let $H_{T}$ be a $(-1)$-compatible component of $G_{\lambda}$. For each vertex $(\tau, \zeta, v, \sigma)$ of $H_{T}$, we define the coefficient $\mathfrak{F}_{v, \tau}$ by the following induction:

1. $\mathfrak{F}_{v, \tau}=1$ if there is no arrow of the form

$$
\left(\tau^{\prime}, \zeta^{\prime}, v^{\prime}, \sigma^{\prime}\right)-s_{i} \longrightarrow(\tau, \zeta, v, \sigma)
$$

in $H_{T}$.

2. $\mathfrak{F}_{v, \tau}=-\frac{\zeta[i]-\zeta[i+1]}{\zeta[i]-\zeta[i+1]+1} \mathfrak{F}_{v^{\prime}, \tau^{\prime}}=-\frac{\zeta^{\prime}[i+1]-\zeta[i]}{\zeta^{\prime}[i+1]-\zeta[i]+1} \mathfrak{F}_{v^{\prime}, \tau^{\prime}}$ if there is an arrow

$$
\left(\tau^{\prime}, \zeta^{\prime}, v^{\prime}, \sigma^{\prime}\right) \rightarrow s_{i} \rightarrow(\tau, \zeta, v, \sigma)
$$

in $H_{T}$.

Again the $\mathfrak{F}_{v, \tau}$ are well defined since the symmetric group acts on the spectral vectors by permuting their components. Define also the antisymmetrization operator

$$
\mathfrak{A}:=\sum_{\omega \in \mathfrak{S}_{N}}(-1)^{\ell(\omega)}(\omega \otimes \omega) .
$$

We will say that a polynomial is antisymmetric if it vanishes under the action of $1-s_{i} \otimes s_{i}$ for each $i<N$.

\section{Theorem 5.12.}

1. Let $H_{T}$ be a connected component of $G_{\lambda}$. For each vertex $(\tau, \zeta, v, \sigma)$ of $H_{T}$, the polynomial $J_{v, \tau} \mathfrak{A}$ equals $J_{\lambda_{T}, \operatorname{std}(T)} \mathfrak{A}$ up to a multiplicative constant.

2. One has $J_{\lambda_{T}, \operatorname{std}(T)} \mathfrak{A} \neq 0$ if and only if $H_{T}$ is (-1)-compatible. 
3. More precisely, when $H_{T}$ is (-1)-compatible, the polynomial

$$
J_{T}^{\prime}=\sum_{(\tau, \zeta, v, \sigma)} \mathfrak{F}_{v, \tau} J_{v, \tau}
$$

is antisymmetric.

Example 5.13. Consider the graph $H_{01}$

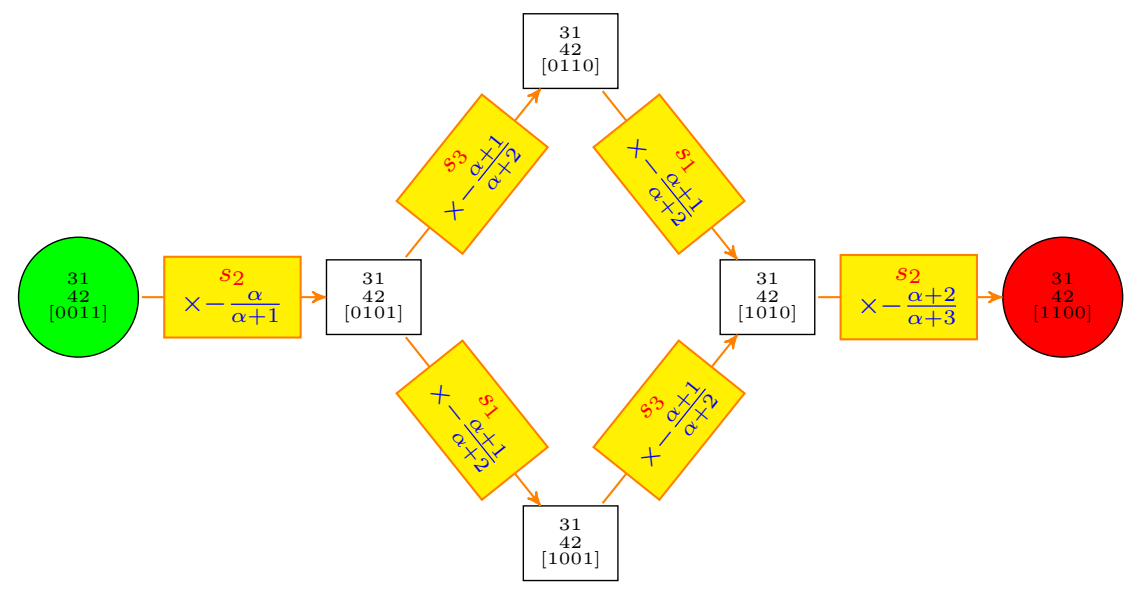

The polynomial

$$
\begin{aligned}
J_{01}^{\prime}= & J_{0011,{ }_{42}^{31}}-\frac{\alpha}{\alpha+1} J_{0101,{ }_{42}^{31}}+\frac{\alpha}{\alpha+2} J_{0110,,_{42}^{31}}+\frac{\alpha}{\alpha+2} J_{1001,{ }_{42}^{31}}-\frac{\alpha(\alpha+1)}{(\alpha+2)^{2}} J_{1010,,_{42}^{31}} \\
& +\frac{\alpha(\alpha+1)}{(\alpha+2)(\alpha+3)} J_{1100,{ }_{42}^{31}}
\end{aligned}
$$

is antisymmetric.

And, as in the symmetric case, one has:

Proposition 5.14. One has

$$
J_{T}=\frac{\# H_{T}}{N !} \mathfrak{F}_{\operatorname{sink}(T)} J_{\operatorname{root}(T)} \cdot \mathfrak{A} .
$$

\subsection{Normalization}

As a consequence of Proposition 4.7, one deduces the following result using Theorems 5.9 and 5.12 .

Corollary 5.15. Let $H_{T}$ be a connected component and $(\tau, \zeta, v, \sigma)$ be a vertex of $H_{T}$. Denote by $\ell_{\tau, v}^{T}$ the length of a path from $\operatorname{root}(T)$ to $(\tau, \zeta, v, \sigma)$. One has,

$$
\left\|J_{v, \tau}\right\|^{2}=(-1)^{\ell_{\tau, v}^{T}} \mathfrak{E}_{v, \tau}^{-1} \mathfrak{F}_{v, \tau}^{-1}\left\|J_{\text {root }}(T)\right\|^{2}
$$

From Theorems 5.9 and 5.12, vector-valued symmetric and antisymmetric Jack polynomials are also pairwise orthogonal.

\section{Proposition 5.16.}

1. Let $H_{T_{1}}$ and $H_{T_{2}}$ be two 1-compatible connected components. If $T_{1} \neq T_{2}$ then $\left\langle J_{T_{1}}, J_{T_{2}}\right\rangle=0$. 
2. Let $H_{T_{1}}$ and $H_{T_{2}}$ be two (-1)-compatible connected components. If $T_{1} \neq T_{2}$ then $\left\langle J_{T_{1}}^{\prime}, J_{T_{2}}^{\prime}\right\rangle=0$.

Proof. It suffices to remark that from Theorem 5.9 (resp. Theorem 5.12) each $J_{T}$ (resp. $J_{T}^{\prime}$ ) is a linear combination of $J_{v, \tau}$ for $(\tau, \zeta, v, \sigma)$ vertex in the connected component $H_{T}$.

In the special cases when $H_{T}$ is \pm 1 -compatible, the value of $\left\|J_{T}\right\|^{2}$ admits a remarkable equality.

Proposition 5.17. One has:

1. If $H_{T}$ is a 1-compatible connected component then

$$
\left\|J_{T}\right\|^{2}=\# H_{T} \mathfrak{E}_{\operatorname{sink}(T)}\left\|J_{\operatorname{root}(T)}\right\|^{2} .
$$

2. If $H_{T}$ is a (-1)-compatible connected component then

$$
\left\|J_{T}^{\prime}\right\|^{2}=\# H_{T} \mathfrak{F}_{\operatorname{sink}(T)}\left\|J_{\operatorname{root}(T)}\right\|^{2} .
$$

Proof. The two cases being very similar, let us only prove the symmetric case. From Proposition 5.11, one has:

$$
\begin{aligned}
\left\|J_{T}\right\|^{2} & =\frac{\# H_{T}}{N !} \mathfrak{E}_{\operatorname{sink}(T)}\left\langle J_{T}, J_{\operatorname{root}(T)} \cdot \mathfrak{S}\right\rangle=\frac{\# H_{T}}{N !} \mathfrak{E}_{\operatorname{sink}(T)} \sum_{\sigma \in \mathfrak{S}_{N}}\left\langle J_{T}, J_{\operatorname{root}(T)}(\sigma \otimes \sigma)\right\rangle \\
& =\# H_{T} \mathfrak{E}_{\operatorname{sink}(T)}\left\|J_{\operatorname{root}(T)}\right\|^{2} .
\end{aligned}
$$

From Corollary 5.15 and Theorem 5.17, one obtains the surprising equalities:

Corollary 5.18. If $H_{T}$ is 1-compatible, one has:

$$
\sum_{(\tau, \zeta, v, \sigma)}(-1)^{\ell_{v, \tau}^{T}} \frac{\mathfrak{E}_{v, \tau}}{\mathfrak{F}_{v, \tau}}=\# H_{T} \mathfrak{E}_{\operatorname{sink}(T)} .
$$

If $H_{T}$ is (-1)-compatible, one has:

$$
\sum_{(\tau, \zeta, v, \sigma)}(-1)^{\ell_{v, \tau}^{T}} \frac{\mathfrak{F}_{v, \tau}}{\mathfrak{E}_{v, \tau}}=\# H_{T} \mathfrak{F}_{\operatorname{sink}(T)} .
$$

Example 5.19. Consider the graph $H_{00}$, the sum (5.3) gives

$$
1+\frac{\alpha+1}{\alpha-1}\left(1+\frac{\alpha}{\alpha-2}\left(2+\frac{\alpha}{\alpha-2}\left(1+\frac{\alpha-1}{\alpha-3}\right)\right)\right)=6 \frac{\alpha(\alpha-1)}{(\alpha-2)(\alpha-3)}
$$

as expected.

\subsection{Symmetric and antisymmetric polynomials with minimal degree}

Since the irreducible characters of $\mathfrak{S}_{N}$ are real it follows that the tensor product of an irreducible module with itself contains the trivial representation exactly once. The tensor product of the module corresponding to a partition $\lambda$ with the module for ${ }^{t} \lambda$ (the transpose) contains the sign representation exactly once. We demonstrate these facts explicitly. Using the concepts from Section 4.1 let

$$
\zeta_{1}=\sum_{\tau \in \mathrm{Tab}_{\lambda}} a(\tau)(\tau \otimes \tau) \in V_{\lambda} \otimes V_{\lambda}
$$


be symmetric with (rational) coefficients $a(\tau)$ to be determined. We impose the conditions $\zeta_{1}\left(s_{i} \otimes s_{i}\right)=\zeta_{1}$ for $i=1, \ldots, N-1$. Fix some $i$ and split the sum as suggested by equation (3.1)

$$
\zeta_{1}=\sum_{b_{\tau}[i]= \pm 1} a(\tau)(\tau \otimes \tau)+\sum_{0<b_{\tau}[i] \leq \frac{1}{2}}\left(a(\tau)(\tau \otimes \tau)+a\left(\tau^{(i, i+1)}\right)\left(\tau^{(i, i+1)} \otimes \tau^{(i, i+1)}\right)\right) .
$$

In the first sum $(\tau \otimes \tau)\left(s_{i} \otimes s_{i}\right)=b_{\tau}[i]^{2}(\tau \otimes \tau)=\tau \otimes \tau$. For the second sum, note that $\tau^{(i, i+1)} s_{i}=\left(1-b_{\tau}[i]^{2}\right) \tau-b_{\tau}[i] \tau^{(i, i+1)}$. Simple computations show that

$$
\begin{gathered}
\left(a(\tau)(\tau \otimes \tau)+a\left(\tau^{(i, i+1)}\right)\left(\tau^{(i, i+1)} \otimes \tau^{(i, i+1)}\right)\right)\left(s_{i} \otimes s_{i}\right) \\
=a(\tau)(\tau \otimes \tau)+a\left(\tau^{(i, i+1)}\right)\left(\tau^{(i, i+1)} \otimes \tau^{(i, i+1)}\right)
\end{gathered}
$$

exactly when $a(\tau)=\left(1-b_{\tau}[i]^{2}\right) a\left(\tau^{(i, i+1)}\right)$. The unique (up to a constant multiple) $\mathfrak{S}_{N^{-}}$ invariant norm on $V_{\tau}$ satisfies $\left\|\tau^{(i, i+1)}\right\|^{2}=\left(1-b_{\tau}[i]^{2}\right)\|\tau\|^{2}$ (see Section 4.3); thus $a(\tau)=$ $c /\|\tau\|^{2}$ for some constant $c$.

Consider the module $V_{t_{\lambda}}$. The transpose map takes each RST $\tau$ with shape $\lambda$ to the $\operatorname{RST}^{t} \tau$ of shape ${ }^{t} \lambda$. Thus $b_{t_{\tau}}[i]=-b_{\tau}[i]$ for $1 \leq i \leq N$. Suppose $0<b_{\tau}[i] \leq \frac{1}{2}$ for some $\tau$ and $i$, then $-\frac{1}{2} \leq b_{\tau}[i]<0$ and the following transformation rules apply:

$$
\begin{aligned}
& { }^{t} \tau s_{i}=b_{t}[i]{ }^{t} \tau+\left(1-b_{t}[i]^{2}\right)\left({ }^{t} \tau^{(i, i+1)}\right), \\
& { }^{t} \tau^{(i, i+1)} s_{i}={ }^{t} \tau-b_{t_{\tau}}[i]{ }^{t} \tau^{(i, i+1)} .
\end{aligned}
$$

Let

$$
\zeta_{\text {det }}=\sum_{\tau \in \operatorname{Tab}_{\lambda}} a(\tau)\left({ }^{t} \tau\right) \otimes \tau \in V_{t_{\lambda}} \otimes V_{\lambda}
$$

be antisymmetric with (rational) coefficients $a(\tau)$ to be determined. We impose the conditions $\zeta_{\text {det }}\left(s_{i} \otimes s_{i}\right)=-\zeta_{\text {det }}$ for $i=1, \ldots, N-1$. Fix some $i$ and write

$$
\zeta_{\mathrm{det}}=\sum_{b_{\tau}[i]= \pm 1} a(\tau)\left({ }^{t} \tau\right) \otimes \tau+\sum_{0<b_{\tau}[i] \leq \frac{1}{2}}\left(a(\tau)\left({ }^{t} \tau\right) \otimes \tau+a\left(\tau^{(i, i+1)}\right)\left({ }^{t} \tau^{(i, i+1)}\right) \otimes \tau^{(i, i+1)}\right) .
$$

In the first $\operatorname{sum}\left(\tau \otimes\left({ }^{t} \tau\right)\right)\left(s_{i} \otimes s_{i}\right)=b_{\tau}[i] b_{t} \tau[i]\left({ }^{t} \tau\right) \otimes \tau=-\left({ }^{t} \tau\right) \otimes \tau$. We find that

$$
\begin{array}{r}
\left(a(\tau)\left({ }^{t} \tau\right) \otimes \tau+a\left(\tau^{(i, i+1)}\right)\left({ }^{t} \tau^{(i, i+1)}\right) \otimes \tau^{(i, i+1)}\right)\left(s_{i} \otimes s_{i}\right) \\
=-\left(a(\tau)\left({ }^{t} \tau\right) \otimes \tau+a\left(\tau^{(i, i+1)}\right)\left({ }^{t} \tau^{(i, i+1)}\right) \otimes \tau^{(i, i+1)}\right)
\end{array}
$$

exactly when $a(\tau)=-a\left(\tau^{(i, i+1)}\right)$.

Thus $a(\tau)=c(-1)^{\operatorname{inv}(\tau)}(\operatorname{recall} \operatorname{inv}(\tau)=\#\{(i, j): 1 \leq i<j \leq N, \operatorname{rw}(i, \tau)>\operatorname{rw}(j, \tau)\}$, and $\left.0<b_{\tau}[i] \leq \frac{1}{2} \operatorname{implies} \operatorname{inv}\left(\tau^{(i, i+1)}\right)=\operatorname{inv}(\tau)+1\right)$.

We can now write down the symmetric and antisymmetric Jack polynomials of lowest degree, by replacing the first factors in $\zeta_{1}$ and $\zeta_{\text {det }}$ by the corresponding polynomials $P_{\tau}(x)$ and $P_{t_{\tau}}(x)$ (as constructed in Section 3). Let $l=\ell(\lambda)={ }^{t} \lambda[1]$.

In the symmetric case let $v=\left[(l-1)^{\lambda[l]},(l-2)^{\lambda[l-1]}, \ldots, 1^{\lambda[2]}, 0^{\lambda[1]}\right]$ (using exponents to indicate the multiplicity of an entry) The corresponding tableau is

$$
T_{1}:=\begin{array}{ccccccc}
l-1 & \ldots & l-1 & & & & (\lambda[l] \times) \\
\vdots & & & \ddots & & \vdots \\
1 & \ldots & \ldots & \ldots & 1 & & (\lambda[2] \times) \\
0 & \ldots & \ldots & \ldots & \ldots & 0 & (\lambda[1] \times)
\end{array}
$$

and $\operatorname{std}\left(T_{1}\right)$ contains the numbers $N, N-1, \ldots, 2,1$ entered row-by-row. 
Example 5.20. If $\lambda=[4,3,2]$ then $v=[221110000]$. The corresponding tableau is

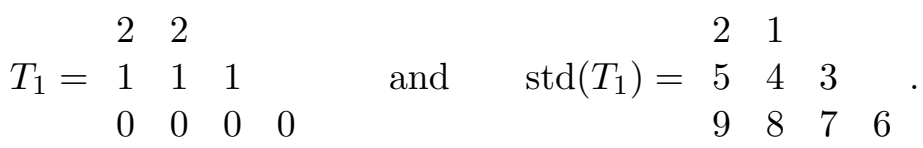

In

$$
\zeta_{1}(x)=\sum_{\tau \in \mathrm{Tab}_{\lambda}} \frac{c}{\|\tau\|^{2}} P_{\tau}(x) \otimes \tau
$$

the monomial $x^{v}$ occurs only when $\tau=\operatorname{std}\left(T_{1}\right)$, with coefficient $c /\left\|\operatorname{std}\left(T_{1}\right)\right\|^{2}$. This polynomial is a multiple of $J_{T_{1}}$ (see Theorem 5.9).

For the antisymmetric case let

$$
\begin{aligned}
& \begin{array}{llll}
0 & 1 & \ldots & \lambda_{l}-1
\end{array} \\
& T_{\text {det }}:=\begin{array}{cccccc}
\vdots & \vdots & & \vdots & \ddots & \\
0 & 1 & \ldots & \lambda_{l}-1 & \ldots & \lambda_{2}-1
\end{array} \\
& \begin{array}{llllllll}
0 & 1 & \ldots & \lambda_{l}-1 & \ldots & \lambda_{2}-1 & \ldots & \lambda_{1}-1
\end{array}
\end{aligned}
$$

Thus $\operatorname{std}\left(T_{\text {det }}\right)=\tau_{\lambda}$ and $v=\left[(\lambda[1]-1)^{t} \lambda[\lambda[1]],(\lambda[1]-2)^{t} \lambda[\lambda[1]-1], \ldots, 0^{t} \lambda[1]\right]$.

Example 5.21. If $\lambda=[4,3,2]$ then ${ }^{t} \lambda=[3,3,2,1]$ and $v=[322111000]$. The corresponding tableau is

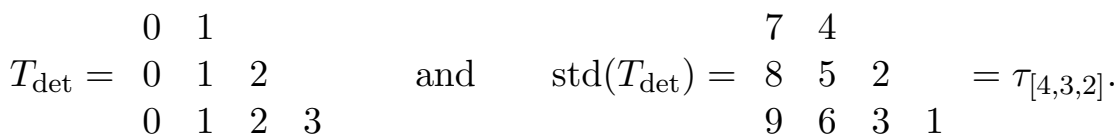

Let

$$
\zeta_{\operatorname{det}}(x)=\sum_{\tau \in \operatorname{Tab}_{\lambda}}(-1)^{\operatorname{inv}(\tau)} P_{t_{\tau}}(x) \otimes \tau .
$$

The monomial $x^{v}$ occurs only in the term $\tau=\tau_{\lambda}$ (see Definition 2.9). This polynomial is a constant multiple of $J_{T_{\text {det }}}^{\prime}$ (see Theorem 5.12).

We summarize the results of this section in the following theorem.

Theorem 5.22. The subspace of $M_{\lambda}$ of the symmetric (resp. antisymmetric) polynomials with minimal degree is spanned by only one generator: the symmetric (resp. antisymmetric) Jack polynomial $J_{T_{1}}\left(\right.$ resp. $\left.J_{T_{\mathrm{det}}}\right)$.

As a consequence one observes a remarkable property.

Corollary 5.23. The Jack polynomial $J_{T_{1}}$ (resp. $\left.J_{T_{\text {det }}}\right)$ is equal to a polynomial which does not depend on the parameter $\alpha$ multiplied by the global multiplicative constant $\mathfrak{E}_{\operatorname{sink}\left(T_{1}\right)}$ (resp. $\left.\mathfrak{F}_{\operatorname{sink}\left(T_{\text {det }}\right)}\right)$.

Proof. The first part of the sentence is a consequence of Theorem 5.22 since the dimension of the space is 1 . The values of the multiplicative constants follow from Theorems 5.9 and 5.12 together with the fact that the coefficient of the leading terms in a Jack polynomials $J_{v, \tau}$ is 1 (see Theorem 4.1).

Note also that $T_{1}$ (resp. $T_{\text {det }}$ ) is not the only tableau for which the corresponding symmetric (resp. antisymmetric) Jack does not depend on $\alpha$ (up to a global multiplicative constant). 
Example 5.24. Consider the partition $\lambda=[221]$ together with the vector $v=[2,1,1,0,0]$. The corresponding symmetric Jack $\frac{1}{E_{21100, \frac{1}{32}}^{1}} J_{\frac{11}{11}}$ does not depend on $\alpha$.

There are two symmetric Jack polynomials in degree 5: $J_{\substack{12 \\ 00}}$ and $J_{31}$. Note that the (non minimal) polynomial $\frac{1}{\mathfrak{E}_{22100, \frac{1}{32}}} J_{\frac{12}{2}}$ does not depend on $\alpha$ whilst the parameter $\alpha$ appears in $\frac{1}{\mathfrak{E}_{31100, \frac{1}{32}}} J_{\frac{11}{54}} \underbrace{3}_{00}$ even after simplifying the expression.

\section{Restrictions}

\subsection{Restrictions on Yang-Baxter graphs}

Consider the operator $\underset{M}{\downarrow}$ acting on the Yang-Baxter graphs $G_{\lambda}$ by producing a new graph $G_{\lambda} \underset{M}{\downarrow}$ following the rules below:

1. Add all the possible edges of the form

$$
(\tau, \zeta,[v[1], \ldots, v[M], 0, \ldots, 0], \sigma) \longrightarrow \Psi^{\prime} \longrightarrow\left(\tau, \zeta^{\prime},[v[2], \ldots, v[M], v[1]+1,0, \ldots, 0], \sigma^{\prime}\right)
$$

More precisely, the action of $\Psi^{\prime}$ on the 4-tuples is given by

$$
\Psi^{\prime}=\Psi s_{N-1} \cdots s_{M} .
$$

2. Suppress the vertices labeled by $(\tau, \zeta, v, \sigma)$ with $v[i] \neq 0$ for some $i>M$, with the associated inward and outward edges.

3. Relabel the remained vertices $(\tau, \zeta, v, \sigma \underset{M}{\downarrow}:=\underset{M}{\underset{M}{\downarrow} \underset{M}{\downarrow}, \underset{M}{\downarrow}, \underset{\downarrow}{\downarrow}, \sigma})$ with

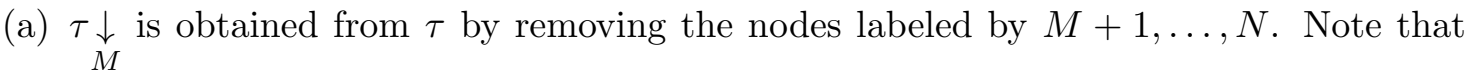 the shape of $\underset{M}{\downarrow}$ could be a skew partition.
(b) $\underset{M}{v \underset{\downarrow}{\downarrow}}=[v[1], \ldots, v[M]]$.
(c) $\underset{M}{\sigma \downarrow}=[\sigma[1], \ldots, \sigma[M]]$.
(d) $\underset{M}{\zeta \downarrow}=\left[\zeta[1]-\mathrm{CT}_{\tau}[M], \ldots, \zeta[M]-\mathrm{CT}_{\tau}[M]\right]$.

4. Relabel by $\Psi$ the edges labeled by $\Psi^{\prime}$.

Example 6.1. Consider the partition $\lambda=21$ and $M=2$, the graph $G_{21}$ in Fig. 1 with edges $\Psi^{\prime}$ added. We obtain the graph $G_{21} \underset{M}{\downarrow}$ (Fig. 7) applying the other rules.

Definition 6.2. A RST $\tau$ has the property $R(M)$ if the removal of the nodes labeled by $M+1, \ldots, N$ in $\tau$ produces a RST whose Ferrers diagram is a partition.

Example 6.3. The RST

$$
\begin{array}{lll}
5 & 2 & \\
7 & 3 & 1 \\
8 & 6 & 4
\end{array}
$$

has the property $R(3)$ while the $\operatorname{RST}_{31}^{2}$ does not have property $R(2)$. 


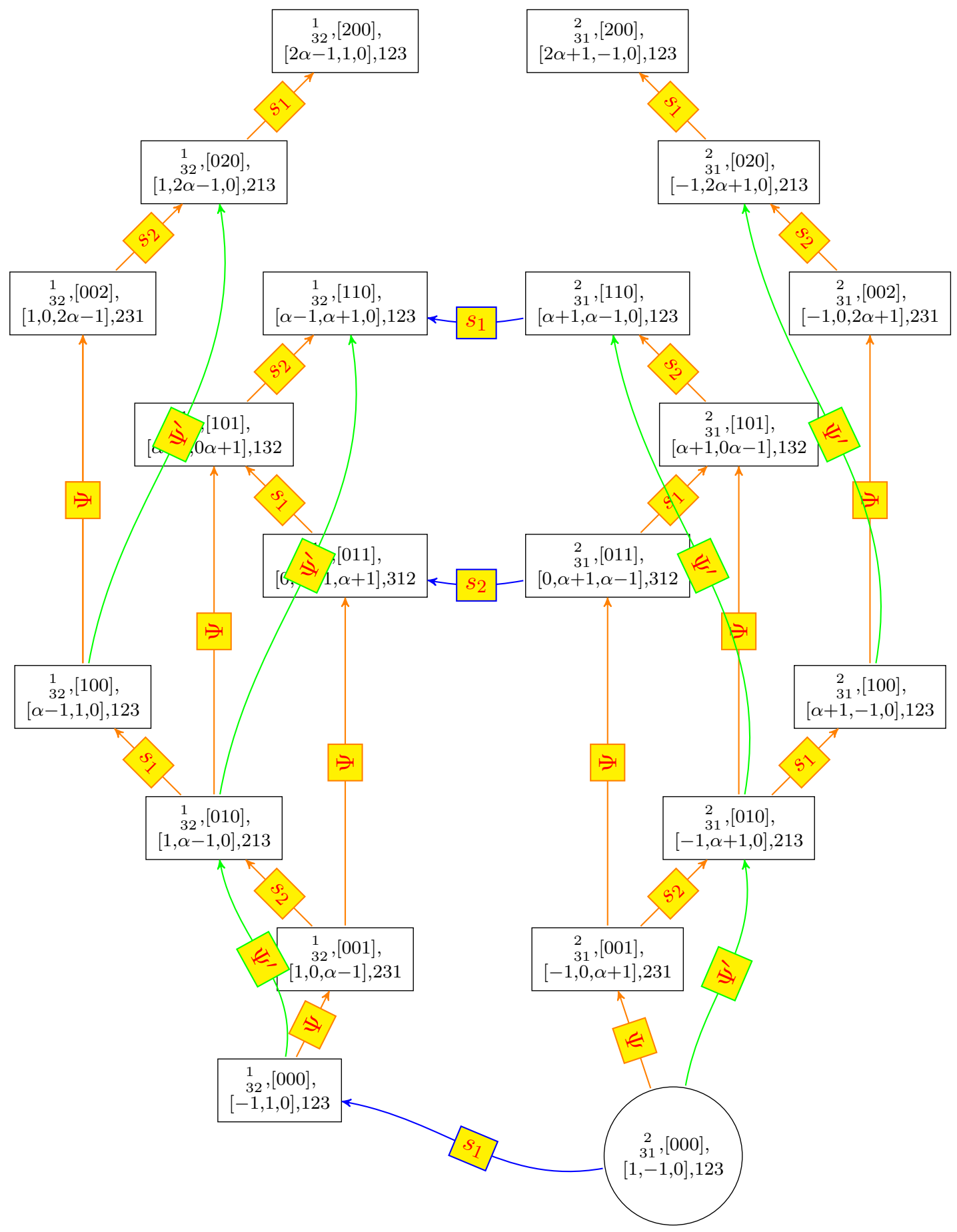

Figure 6. The first vertices of the graph $G_{21}$ with edges $\Psi^{\prime}$ for $M=2$.

Denote by $\overline{G_{\tau}}$ the subgraph of $G_{\lambda}$ whose root is $\tau$. In particular, one has

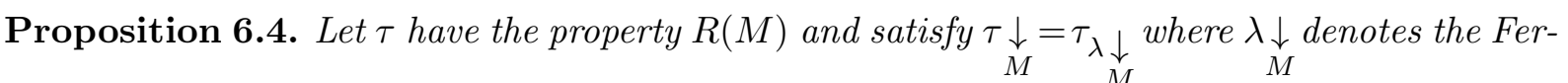

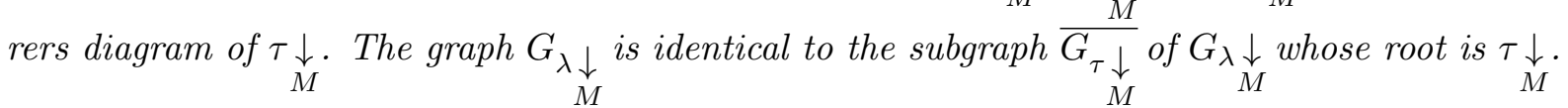

Proof. Obviously, since the Ferrers diagram of $\underset{M}{\tau \underset{M}{\downarrow}}$ is a partition, all the spectral vectors $\zeta$ labeling the vertices of $\overline{G_{\tau \downarrow}}$ are obtained by subtracting the same integer (that is $\mathrm{CT}_{\tau}[M]$ ) 


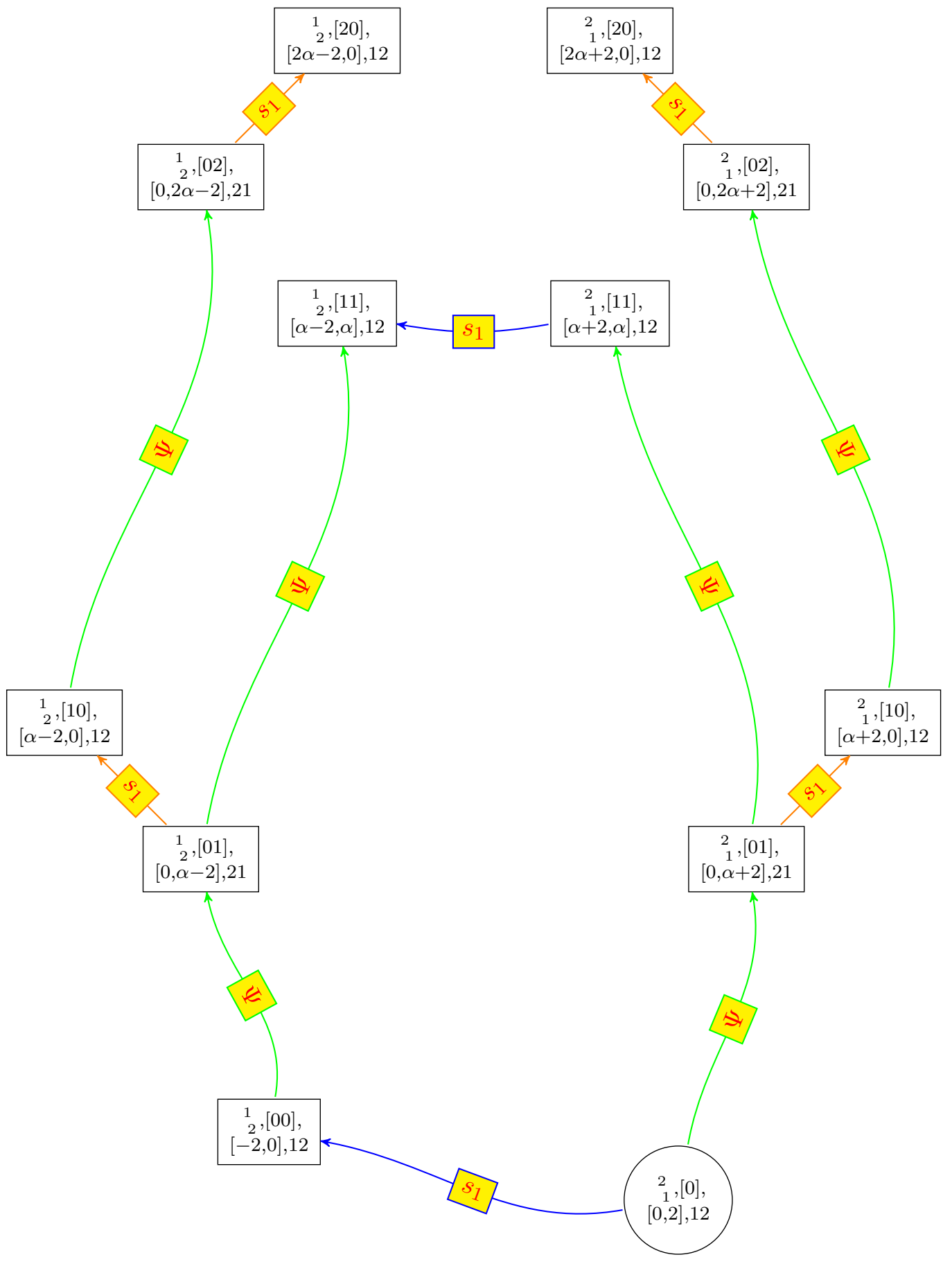

Figure 7. The first vertices of the graph $G_{21} \downarrow$.

from the corresponding spectral vector in $G_{\lambda}$. It follows that the action of the $s_{i}$ permutes the components of the spectral vectors in $\overline{G_{\tau} \downarrow}$.

Let $\mathbf{v}^{\prime}=\left(\tau^{\prime}, \zeta^{\prime},\left[v^{\prime}[1], \ldots, v^{\prime}[M], 0, \ldots, 0\right], \sigma^{\prime}\right)$ be a vertex of $\bar{G}_{\tau}$. Let us prove by induction on the length of a path from the root to $\mathbf{v}^{\prime}$ that

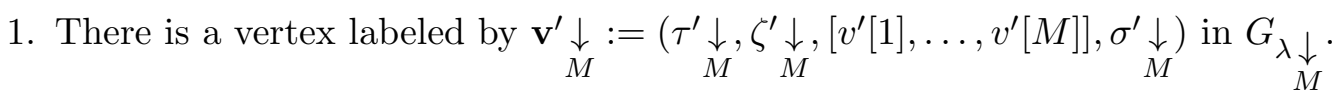


2. If there is a non affine edge labeled by $s_{i}$ with $i<M$ from

$$
\mathbf{v}^{\prime \prime}=\left(\tau^{\prime \prime}, \zeta^{\prime \prime},\left[v^{\prime \prime}[1], \ldots, v^{\prime \prime}[M], 0, \ldots, 0\right], \sigma^{\prime \prime}\right)
$$

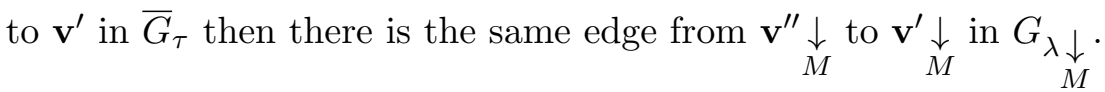

3. If there is an edge from $\mathbf{v}^{\prime \prime}=\left(\tau^{\prime \prime}, \zeta^{\prime \prime},\left[v^{\prime \prime}[1], \ldots, v^{\prime \prime}[M], 0, \ldots, 0\right], \sigma^{\prime \prime}\right)$ to $\mathbf{v}^{\prime}$ in $\overline{G_{\tau}}$ labeled by

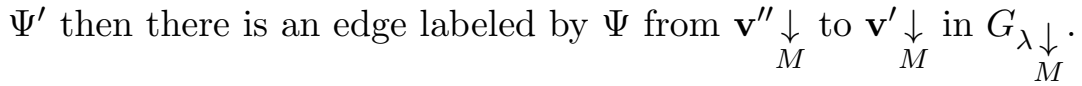

First, observe that if $\tau^{\prime}=\tau$ and $v^{\prime}[i]=0$ for each $i$ (i.e. $\mathbf{v}^{\prime}$ is a component of the label of the root of $\overline{G_{\tau}}$ ) then the construction gives, straightforwardly the result.

Suppose that there is a non affine edge

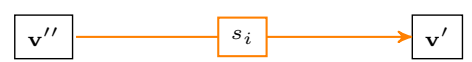

in $\overline{G_{\tau}}$. By induction $\mathbf{v}_{M}^{\prime \prime} \underset{M}{\downarrow}$ labels a vertex of $\underset{\substack{\downarrow \\ G^{\downarrow}}}{ }$. We verify that

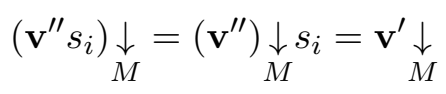

Hence, $\mathbf{v}^{\prime} \underset{M}{\downarrow}$ labels a vertex of $\underset{\substack{\downarrow \\ G^{\downarrow}}}{ }$ and the assertion (2) is recovered.

Suppose now, that there is a affine edge

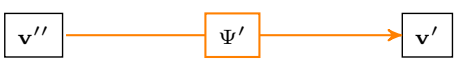

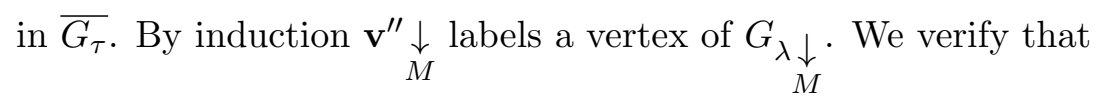

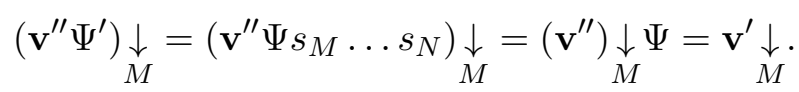

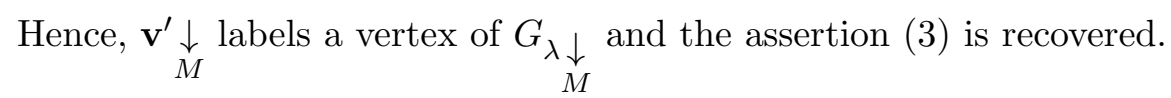

Conversely, if $\mathbf{v}^{\prime}$ labels a vertex of $G_{\lambda \downarrow}$, there exists a vertex labeled by $\mathbf{v}^{\prime(N)}$ in $\overline{G_{\tau}}$ verifying $\mathbf{v}^{(N)} \underset{M}{\downarrow}=\mathbf{v}^{\prime}$. Indeed, suppose $\mathbf{v}^{\prime}=\left(\tau^{\prime}, \zeta^{\prime}, v^{\prime}, \sigma^{\prime}\right)$ then $\mathbf{v}^{\prime(N)}=\left(\tau^{\prime(N)}, \zeta^{\prime(N)}, v^{(N)}, \sigma^{\prime(N)}\right)$, where $\tau^{\prime(N)}$ is obtained from $\tau^{\prime}$ by adding the nodes of $\tau$ labeled by $M+1, \ldots, N, v^{(N)}=$ $\left[v^{\prime}[1], \ldots, v^{\prime}[M], 0, \ldots, 0\right], \zeta^{\prime(N)}=\zeta_{\left.v^{\prime(N)}, \tau^{\prime}(N)\right)}$ and $\sigma^{\prime(N)}=\sigma_{v^{\prime}(N)}$. Furthermore if $\mathbf{v}^{\prime} s_{i}=\mathbf{v}^{\prime \prime}$ then $\mathbf{v}^{\prime(N)} s_{i}=\mathbf{v}^{\prime \prime(N)}$ and if $\mathbf{v}^{\prime} \Psi=\mathbf{v}^{\prime \prime}$ then $\mathbf{v}^{\prime(N)} \Psi^{\prime}=\mathbf{v}^{\prime \prime(N)}$. This concludes the proof.

Example 6.5. Consider in Fig. 8 the restriction problem for

$$
\tau=\begin{array}{lll}
5 & 2 & \\
7 & 3 & 1 \\
8 & 6 & 4
\end{array}
$$

and $M=3$. The subgraph of $\begin{array}{rrrr}G & & & \\ 5 & 2 & \\ 7 & 3 & 1 \\ 8 & 6 & 4\end{array}$ obtained using only the root and the arrows labeled by $\Psi^{\prime}, s_{1}$ and $s_{2}$ is isomorphic to the graph $\overline{G_{2}}$ (see Fig. 1). 


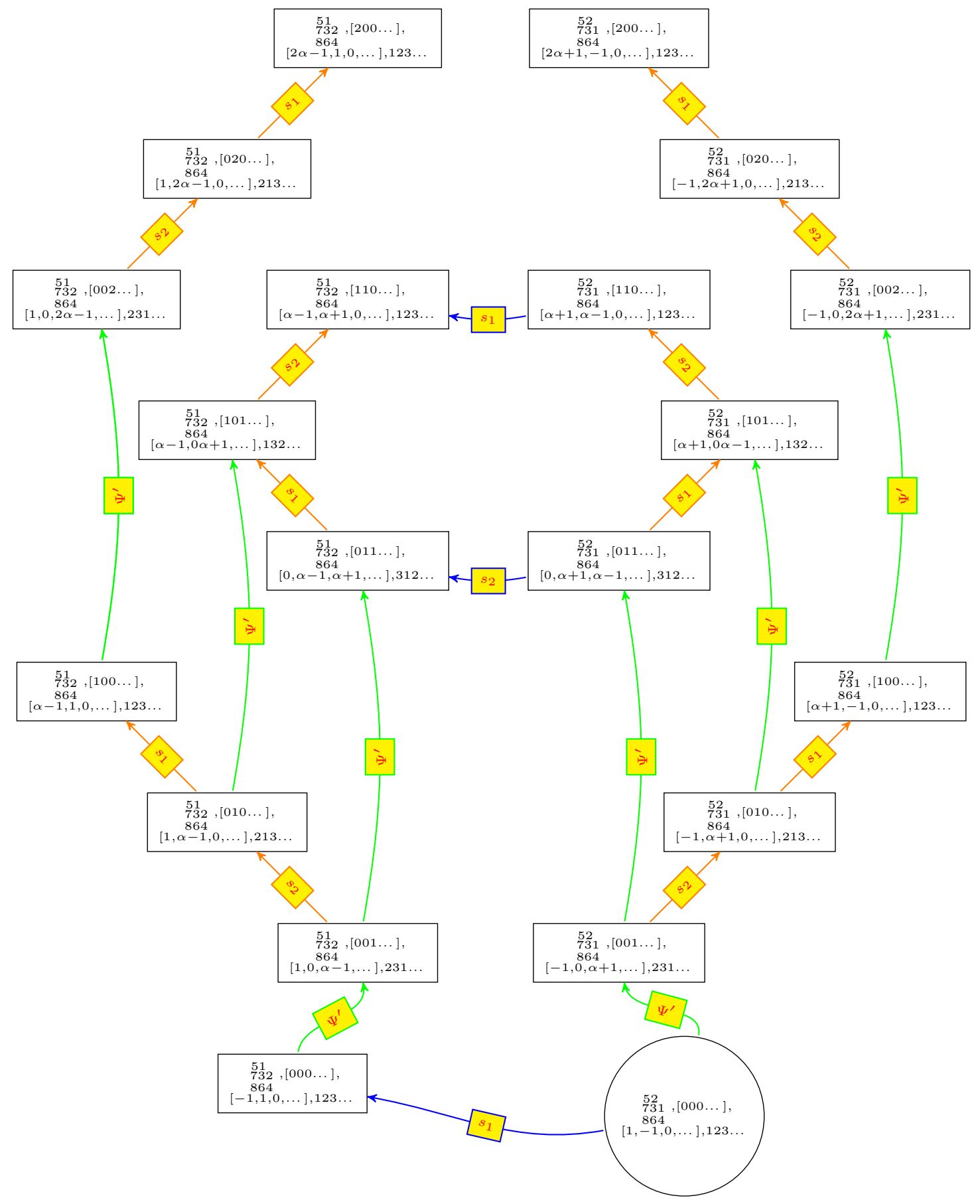

Figure 8. The first vertices of the subgraph of $G_{332}$ associated to the restriction for $M=3$ whose root is $\underset{732}{531}$ with the edges $\Psi^{\prime}$ added.

\subsection{Restrictions on tableaux}

In the sequel, as in [15], we will denote a skew partition by $\lambda / \mu$.

Let $\tau$ be a RST of shape $\lambda=\left[\lambda_{1}, \ldots, \lambda_{k}\right]$ and $M<N$. Consider $P_{\tau}$ as a polynomial in $\mathbb{C}\left[t_{1}, \ldots, t_{M}\right]\left[t_{M+1}, \ldots, t_{N}\right]$. Let $\mu$ the sub-partition of $\lambda$ which is the shape of the RST obtained by removing the nodes labeled by $1, \ldots, M$ in $\tau$ and denote by $\tau^{(M)}$ the associated RST. Consider also the skew-RST $\tau \downarrow$ of shape $\lambda / \mu$ obtained by removing the nodes labeled 
$M+1, \ldots, N$ in $\tau$. Let $C(\tau, M):=\left\{\rho: \rho^{(M)}=\tau^{(M)}\right\}$ and $T(\tau, M):=\left\{\begin{array}{c}\downarrow \\ M\end{array}: \rho \in C(\rho, M)\right\}$.

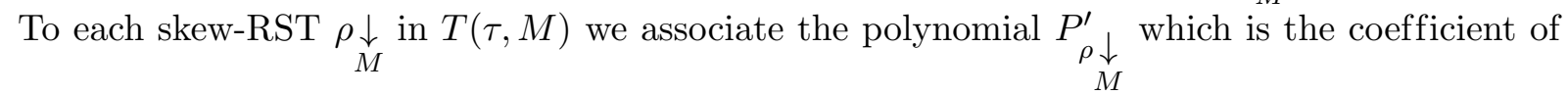
the monomial $\prod_{i=M+1}^{N} t_{i}^{\lambda_{\mathrm{cl}(i, \rho)}-\mathrm{rw}(i, \rho)}$ in $P_{\rho}$ (recall that $\operatorname{cl}(i, \tau)$ is the column of $\tau$ containing $i$ and $\operatorname{rw}(i, \tau)$ is the row of $\tau$ containing $i)$.

Example 6.6. Consider $\tau=\begin{array}{ll}2 & 1 \\ 4 & 3\end{array}$ and $M=3$. Then $\tau^{(3)}={ }_{5}^{4}, \underset{3}{\tau \downarrow}=\begin{aligned} & 2 \\ & 1\end{aligned}$ ，

$$
C(\tau, 3)=\left\{\begin{array}{llllll}
3 & & 2 & & 1 & \\
4 & 1, & 4 & 1 & 4 & 2 \\
5 & 2 & 5 & 3 & 5 & 3
\end{array}\right\}
$$

and

$$
T(\tau, 3)=\left\{\begin{array}{llllll}
3 & & 2 & & 1 & \\
& 1, & & 1, & & 2 \\
2 & & 3 & & 3
\end{array}\right\} .
$$

The polynomial $P_{2}^{\prime} \quad$ is the coefficient of $t_{5}^{2} t_{4}$ in $P_{2}$.

$$
\begin{array}{lll}
1 & 4 & 1 \\
3 & 5 & 3
\end{array}
$$

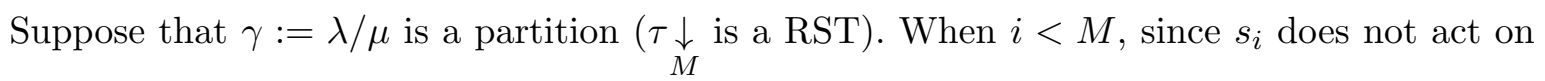
the variables $t_{M+1}, \ldots, t_{N}$, the Murphy rules (equation 3.1) give

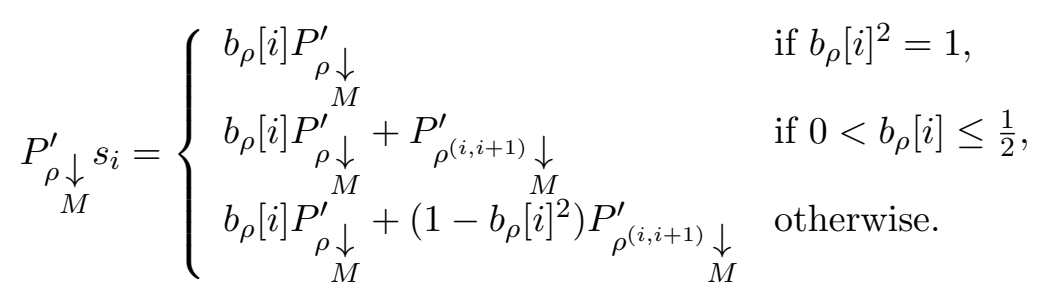

Since the action $s_{i}(i<M)$ on the RST commutes with the restriction $\underset{M}{\downarrow}$, one has $\underset{\rho^{(i, i+1)} \underset{M}{\downarrow}}{P^{\prime}}=$ $P_{\substack{(\rho \downarrow j) \\ M}}^{\prime(i, i+1)}$ and Proposition 3.1 and equation (6.1) imply that the polynomials $\underset{\rho \downarrow}{P^{\downarrow}}{ }^{\prime}$ are simultaneous eigenfunctions of the Jucys-Murphy operators

$$
\omega_{i}^{(M)}=\sum_{i+1}^{M} s_{i j}
$$

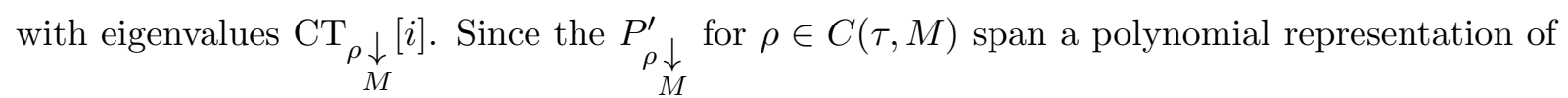

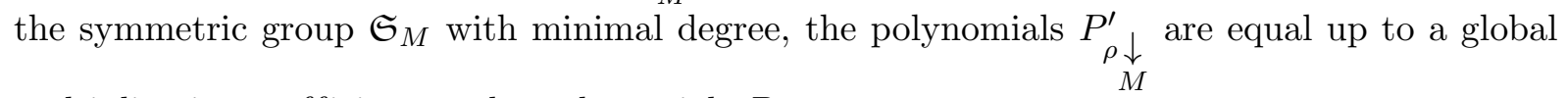
multiplicative coefficient to the polynomials $P_{\rho \downarrow}$.

To summarize:

Proposition 6.7. When $\underset{M}{\downarrow}$ is a RST, the coefficient of $\prod_{i=M+1}^{N} t_{i}^{\lambda_{\mathrm{cl}(i, \tau)}-\mathrm{rw}(i, \tau)}$ in $P_{\tau}$ is propor-

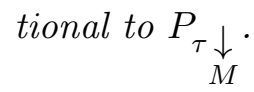


Example 6.8. The coefficient of $t_{9}^{2} t_{8}^{2} t_{7} t_{6}$ in $P_{\substack{51 \\ 732 \\ 9864}}$ equals

$$
\frac{1}{6} t_{1}-\frac{1}{12} t_{2}-\frac{1}{12} t_{3}=\frac{1}{6} P_{32}
$$

\subsection{Restrictions on Jack polynomials}

Consider the linear map $\underset{M}{\underset{\downarrow}{\downarrow}}$ which sends each $x_{i}$ to 0 when $i>M$ and each $\tau$ to $\underset{M}{\downarrow}$.

Theorem 6.9. Let $\tau$ have property $R(M)$. Then

$$
J_{[v[1], \ldots, v[M], 0, \ldots, 0], \tau \underset{M}{\downarrow}}^{\downarrow}=J_{[v[1], \ldots, v[M]], \tau \underset{M}{\downarrow}}^{\underset{\downarrow}{\downarrow}}
$$

Proof. From Proposition 6.4 the graphs $\overline{G_{\tau} \underset{M}{\downarrow}}$ and $\underset{\substack{\text { shape }(\tau \downarrow) \\ \downarrow}}{ }$ are the same. Remark that:

1. The result is correct for the roots:

$$
J_{\left([0, \ldots, 0], \tau_{\text {root }}\right) \underset{M}{\downarrow}}^{\underset{\downarrow}{\downarrow}}=J_{[0, \ldots, 0], \tau_{\text {root }} \underset{M}{\downarrow}}
$$

where $\tau_{\text {root }}$ denotes the RST obtained from $\tau$ by replacing subtableau constituted with the nodes labeled $1, \ldots, M$ by $\tau_{\text {shape }(\tau \underset{M}{\downarrow})}$.

2. The action of the edges are compatible with the restriction:

(a) The non-affine edges: We use only the difference between the content of two boxes.

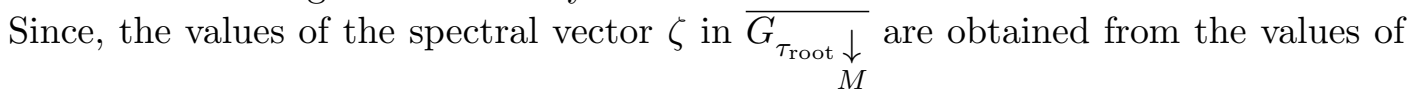
the spectral vector in $G_{\tau_{\text {root }}}$ by adding to each component the same integer. Hence, the differences are the same and then the action of the non-affine edges are the same.

(b) The affine edges: One has to verify that

$$
\begin{aligned}
& J_{[v[1], \ldots, v[M], 0, \ldots, 0], \tau} \Psi_{N}\left(s_{N-1} \otimes s_{N-1}+(*)\right) \cdots\left(s_{M} \otimes s_{M}+(*)\right) \underset{M}{\downarrow} \\
& \quad=J_{[v[1], \ldots, v[M], 0, \ldots, 0], \tau} \underset{M}{\downarrow} \Psi_{M},
\end{aligned}
$$

where $(*)$ denote the correct rational numbers corresponding to the edges of the Yang-Baxter graph and $\Psi_{M}$ means that one applies the operator $\Psi$ for an alphabet of size $M$. This identity is easy to obtained from the construction: since $\Psi$ gives a polynomial whose a factor is $x_{N}$, the only non vanishing part of

$$
J_{[v[1], \ldots, v[M], 0, \ldots, 0], \tau} \Psi_{N}\left(s_{N-1} \otimes s_{N-1}+(*)\right) \cdots\left(s_{M} \otimes s_{M}+(*) \underset{M}{\downarrow}\right.
$$

is

$$
J_{[v[1], \ldots, v[M], 0, \ldots, 0], \tau} \Psi_{N}\left(s_{N-1} \cdots s_{M} \otimes s_{N-1} \cdots s_{M}\right) \underset{M}{\underset{\downarrow}{\downarrow}}=J_{[v[1], \ldots, v[M], 0, \ldots, 0], \tau} \Psi_{M} \underset{M}{\downarrow},
$$

the last part of the proof follows from the commutation $\underset{M}{\Psi_{M}} \underset{M}{\downarrow} \underset{M}{\downarrow} \Psi_{M}$.

This shows that the polynomials $J_{[v[1], \ldots, v[M], 0, \ldots, 0], \tau} \underset{M}{\downarrow}$ are inductively generated following the same Yang-Baxter graph as the polynomials $J_{[v[1], \ldots, v[M]], \underset{M}{\downarrow}}$ with the same initial conditions. Hence $J_{[v[1], \ldots, v[M], 0, \ldots, 0], \tau}^{\underset{M}{\downarrow}} \underset{[v[1], \ldots, v[M]], \tau \underset{M}{\downarrow}}{\underset{1}{\downarrow}}$ as expected. 


\section{Shifted vector-valued Jack polynomials}

\subsection{Knop and Sahi operators for vector-valued polynomials}

Let us define the following operators which are the vector-valued versions of the operators defined in [9]:

1. $\varsigma_{i}:=\partial_{i} \otimes 1+s_{i} \otimes s_{i}$, where $\partial_{i}:=\partial_{i, i+1}=\left(1-s_{i}\right) \frac{1}{x_{i}-x_{i+1}}$ is a divided difference.

2. Denote by $\Phi$ the operator sending each $x_{i}$ to $x_{i-1}$ for $i>1$ and $x_{1}$ to $x_{N}-\alpha$ and $T:=\Phi \otimes\left(s_{1} s_{2} \cdots s_{N-1}\right)$.

3. $\hat{\Psi}:=T\left(x_{N}+N-1\right)$.

Proposition 7.1. The operators $\varsigma_{i}$ satisfy the braid relations

$$
\varsigma_{i} \varsigma_{i+1} \varsigma_{i}=\varsigma_{i+1} \varsigma_{i} \varsigma_{i+1}, \quad \varsigma_{i} \varsigma_{j}=\varsigma_{j} \varsigma_{i}, \quad|i-j|>1,
$$

and the relations between the $\varsigma_{i}$ and the multiplication by the indeterminates are given by the Leibniz rules:

$$
x_{i} \varsigma_{i}=\varsigma_{i+1} x_{i}+1, \quad x_{i+1} \varsigma_{i}=\varsigma_{i} x_{i}-1, \quad x_{j} \varsigma_{i}=\varsigma_{i} x_{j}, \quad j \neq i, i+1 .
$$

Proof. The Leibniz rules are straightforward from the definition while the braid relations are a direct consequence of

1) the braid relations on the $s_{i}$ and the braid relations on the $\partial_{i}$,

2) $\partial_{i+1} \partial_{i} s_{i}+s_{i+1} \partial_{i} \partial_{i+1}=\partial_{i} s_{i+1} \partial_{i}$,

3) $\partial_{i} \partial_{i+1} s_{i}+s_{i} \partial_{i+1} \partial_{i}=\partial_{i+1} s_{i} \partial_{i+1}$,

4) $s_{i} \partial_{i+1} s_{i}=s_{i+1} \partial_{i} s_{i+1}$,

5) $\partial_{i} s_{i+1} s_{i}=s_{i+1} s_{i} \partial_{i+1}$,

6) $s_{i} s_{i+1} \partial_{i}=\partial_{i+1} s_{i} s_{i+1}$.

Since the $\varsigma_{i}$ verify the braid relations, they realize the braid group: given a permutation $\omega \in \mathfrak{S}_{N}$ and a reduced decomposition $\omega=s_{i_{1}} \cdots s_{i_{k}}$, the product $\varsigma_{i_{1}} \cdots \varsigma_{i_{k}}$ is independent of the choice of the reduced decomposition. We will denote $\varsigma_{\omega}:=\varsigma_{i_{1}} \cdots \varsigma_{i_{k}}$.

Furthermore, the algebra generated by the $\varsigma_{i}$ and the $x_{j}$ is isomorphic to the degenerate Hecke affine algebra generated by the operators $s_{i}+\partial_{i}$ and the variables.

Our goal is to find a basis of simultaneous eigenvectors of the following operators

$$
\hat{\xi}_{i}:=x_{i}+N-1-\varsigma_{i} \cdots \varsigma_{N-1} \hat{\Psi} \varsigma_{1} \cdots \varsigma_{i-1} .
$$

These operators commute and play the role of Cherednik elements for our representation of the degenerate Hecke affine algebra. As a consequence, one has the following relations:

\section{Proposition 7.2.}

1. $\varsigma_{i} \hat{\xi}_{i+1}=\hat{\xi}_{i} \varsigma_{i}-1$,

2. $\varsigma_{i} \hat{\xi}_{i}=\hat{\xi}_{i+1} \varsigma_{i}+1$,

3. $\varsigma_{i} \hat{\xi}_{j}=\hat{\xi}_{j} \varsigma_{i}$ for $j \neq i, i+1$,

4. $\hat{\Psi} \hat{\xi}_{i}=\hat{\xi}_{i-1} \hat{\Psi}$ for $i \neq 1$,

5. $\hat{\Psi} \hat{\xi}_{1}=\left(\hat{\xi}_{N}-\alpha\right) \hat{\Psi}$. 
Furthermore, the RST are simultaneous eigenfunctions of the operators $\hat{\xi}_{i}$. More precisely

\section{Proposition 7.3.}

$$
\tau \hat{\xi}_{i}=\mathrm{CT}_{\tau}[i] \tau .
$$

Proof. The action of $\hat{\xi}_{i}$ on polynomials with degree 0 in the $x_{i}$ equals the action of the operators $\tilde{\xi}_{i}$. Hence, the result follows from the non-shifted version of the equality (Proposition 3.13).

Straightforwardly, the operators $\varsigma_{i}$ and $\hat{\Psi}$ are compatible with the leading monomials in the following sense:

Proposition 7.4. Suppose that $P$ a polynomial such that its highest degree component has the leading monomial $x^{v, \tau}$ then

1. If $v[i]<v[i+1]$ then the highest degree component of $P \varsigma_{i}$ has the leading monomial $x^{v s_{i}, \tau}$.

2. The highest degree component of $P \hat{\Psi}$ has the leading monomial $x^{v \Psi, \tau}$.

\subsection{The Yang-Baxter graph}

Let $\lambda$ be a partition and $G_{\lambda}$ the associated graph. We construct the set of the polynomials $\left(\hat{J}_{\mathfrak{P}}\right)_{\mathfrak{P} \text { path in } G_{\lambda}}$ using the following recurrence rules:

1. $\hat{J}_{[]}:=1 \otimes \tau_{\lambda}$.

2. If $\mathfrak{P}=\left[a_{1}, \ldots, a_{k-1}, s_{i}\right]$ then

$$
\hat{J}_{\mathfrak{P}}:=\hat{J}_{\left[a_{1}, \ldots, a_{k-1}\right]}\left(\varsigma_{i}+\frac{1}{\zeta[i+1]-\zeta[i]}\right),
$$

where the vector $\zeta$ is defined by

$$
\left(\tau_{\lambda}, \mathrm{CT}_{\tau_{\lambda}}, 0^{N},[1,2, \ldots, N]\right) a_{1} \cdots a_{k-1}=(\tau, \zeta, v, \sigma) .
$$

3. $\mathfrak{P}=\left[a_{1}, \ldots, a_{k-1}, \Psi\right]$ then then

$$
\hat{J}_{\mathfrak{P}}=\hat{J}_{\left[a_{1}, \ldots, a_{k-1}\right]} \hat{\Psi} \text {. }
$$

As expected one obtains

Theorem 7.5. Let $\mathfrak{P}=\left[a_{0}, \ldots, a_{k}\right]$ be a path in $G_{\lambda}$ from the root to $(\tau, \zeta, v, \sigma)$. The polynomial $\hat{J}_{\mathfrak{P}}$ is a simultaneous eigenfunction of the operators $\hat{\xi}_{i}$ whose leading monomial in the highest degree component is $x^{v, \tau}$. Furthermore, the eigenvalue of $\hat{\xi}_{i}$ associated to $\hat{J}_{\mathfrak{P}}$ equals $\zeta[i]$.

Consequently $\hat{J}_{\mathfrak{P}}$ does not depend on the path, but only on the end point $(\tau, \zeta, v, \sigma)$, and will be denoted by $\hat{J}_{v, \tau}$. The family $\left(\hat{J}_{v, \tau}\right)_{v, \tau}$ forms a basis of $M_{\lambda}$ of simultaneous eigenfunctions of the Cherednik operators.

Furthermore, if $\mathfrak{P}$ leads to $\varnothing$ then $\hat{J}_{\mathfrak{P}}=0$.

Proof. The proof goes as in Theorem 4.1 using respectively Propositions 7.2, 7.3 and 7.4 instead of Propositions 3.14, 3.13 and 3.8.

In consequence, we will consider the family of polynomials $\left(\hat{J}_{v, \tau}\right)_{v, \tau}$ indexed by pairs $(v, \tau)$ where $v \in \mathbb{N}^{N}$ is a weight and $\tau$ is a tableau. 


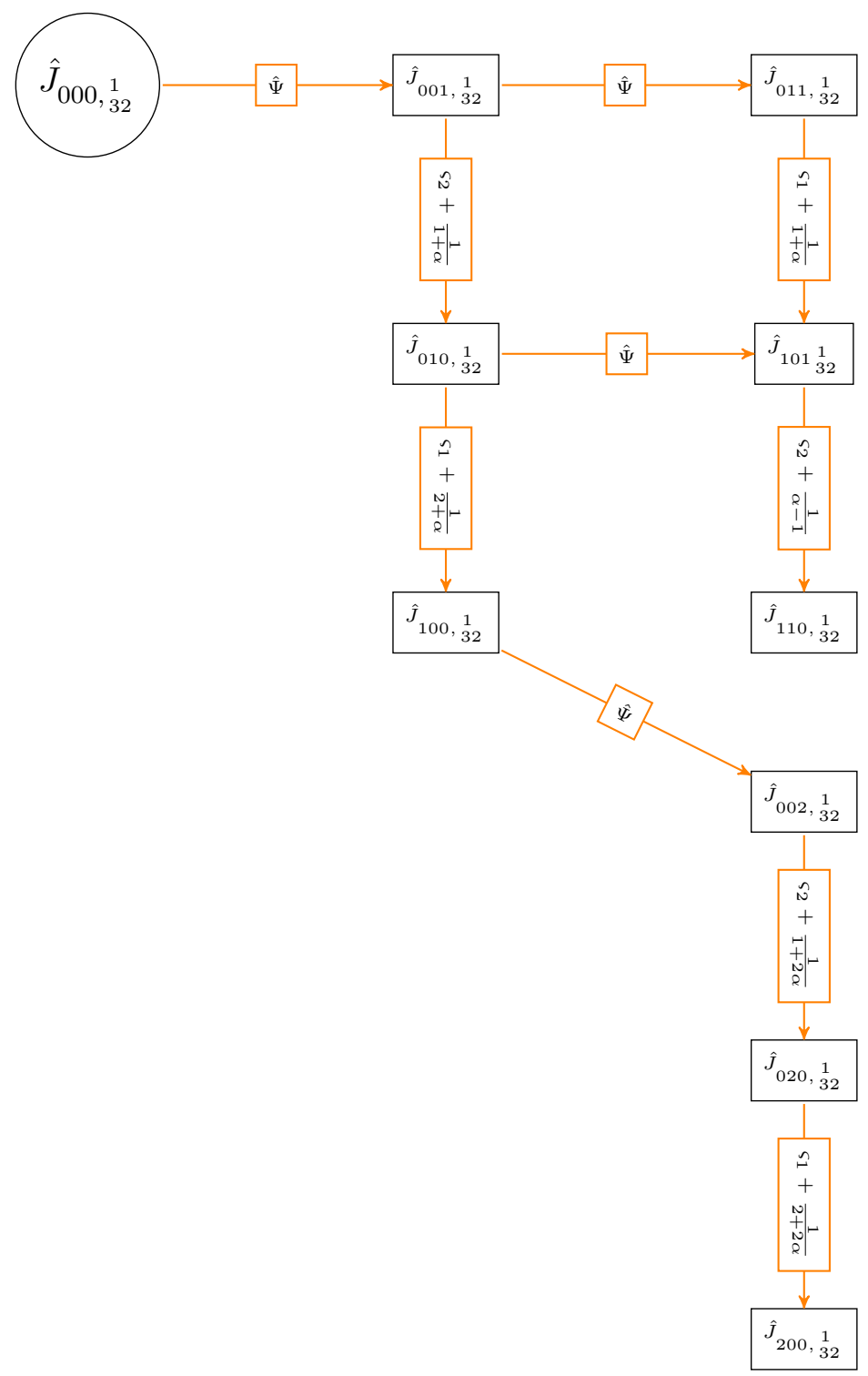

Figure 9. First values of $\hat{J}_{v,{ }_{31}^{2}}$.

Example 7.6. Let again $\tau=\begin{aligned} & 2 \\ & 3\end{aligned} \quad 1$ and consider the Yang-Baxter Graph $G_{31}$ (see Fig. 9).

The eigenvalues of $\tau$ are $[1,-1,0]$. Let $\tau^{\prime}=\begin{aligned} & 1 \\ & 3\end{aligned} 2$, one has

$$
\hat{J}_{[001], \tau}=\tau \hat{\Psi}=\left(x_{3}+2\right) \otimes\left(-\frac{1}{2} \tau+\tau^{\prime}\right)
$$

with associated eigenvalues $[-1,0, \alpha+1]$ and

$$
\begin{aligned}
\hat{J}_{[010], \tau} & =\hat{J}_{[001], \tau}\left(\left(s_{2}+\partial_{2,3}\right) \otimes s_{2}+\frac{1}{\alpha+1}\right) \\
& =\frac{1}{2} \frac{\left(3 \alpha+1+x_{2}+\alpha x_{2}-x_{3}\right)}{\alpha+1} \tau+\frac{\left(x_{3}+3+x_{2}+\alpha x_{2}+\alpha\right)}{\alpha+1} \tau^{\prime}
\end{aligned}
$$

with eigenvalues $[-1, \alpha+1,0]$. 
The non-shifted vector-valued Jack polynomials can be recovered easily from the shifted one:

Proposition 7.7. The restriction of $\hat{J}_{v, \tau}$ to its component of top degree equals $J_{v, \tau}$.

Proof. It suffices to remark that $\varsigma_{i}=s_{i} \otimes s_{i}+\varsigma_{i}^{-}$and $\hat{\Psi}=\Psi+\Psi^{-}$where $\varsigma^{-}$and $\Psi^{-}$are operators which decrease the degree in the $x_{j}$. Hence, one computes the component of the top degree of $\hat{J}_{v, \tau}$ following the Yang-Baxter graph replacing $\varsigma_{i}$ by $s_{i} \otimes s_{i}$ and $\hat{\Psi}$ by $\Psi$, that is the Yang-Baxter graph which was used to obtain the polynomials $J_{v, \tau}$. This ends the proof.

\subsection{Symmetrization, antisymmetrization}

We will say that a polynomial is symmetric if it is invariant under the action of $\varsigma_{i}$ for each $i<N-1$. Denote also $\hat{\mathfrak{S}}=\sum_{\omega \in \mathfrak{S}_{N}} \varsigma_{\omega}$. As for non shifted Jack, one has:

\section{Theorem 7.8.}

1. Let $H_{T}$ be a connected component of $G_{\lambda}$. For each vertex $(\tau, \zeta, v, \sigma)$ of $H_{T}$, the polynomial $\hat{J}_{v, \tau} \hat{\mathfrak{S}}$ equals $\hat{J}_{\lambda_{T}, \operatorname{std}(T)} \hat{\mathfrak{S}}$ up to a multiplicative constant.

2. One has $\hat{J}_{\lambda_{T}, \operatorname{std}(T)} \hat{\mathfrak{S}} \neq 0$ if and only if $H_{T}$ is 1-compatible.

3. More precisely, when $H_{T}$ is 1-compatible, the polynomial

$$
\hat{J}_{T}=\sum_{(\tau, \zeta, v, \sigma)} \mathfrak{E}_{v, \tau} \hat{J}_{v, \tau}
$$

is symmetric.

Proof. The proof is identical to the non-shifted case.

In the same way, for the antisymmetrization, denoting $\hat{\mathfrak{A}}:=\sum_{\omega \in \mathfrak{S}_{N}}(-1)^{\ell(\omega)} \varsigma_{\omega}$, we have:

\section{Theorem 7.9.}

1. Let $H_{T}$ be a connected component of $G_{\lambda}$. For each vertex $(\tau, \zeta, v, \sigma)$ of $H_{T}$, the polynomial $\hat{J}_{v, \tau} \hat{\mathfrak{A}}$ equals $\hat{J}_{\lambda_{T}, \operatorname{std}(T)} \hat{\mathfrak{A}}$ up to a multiplicative constant.

2. One has $\hat{J}_{\lambda_{T}, \operatorname{std}(T)} \hat{\mathfrak{A}} \neq 0$ if and only if $H_{T}$ is $(-1)$-compatible.

3. More precisely, when $H_{T}$ is (-1)-compatible the polynomial

$$
\hat{J}_{T}^{\prime}=\sum_{(\tau, \zeta, v, \sigma)} \mathfrak{F}_{v, \tau} \hat{J}_{v, \tau}
$$

is antisymmetric.

\subsection{Propagation of vanishing properties}

Some phenomena of propagation of vanishing properties can be deduced from the classical case (see [14]).

Lemma 7.10 (Lascoux). Let $f(x, y)$ be a function of two variables. Suppose that $f(b, a)=0$ with $a \neq b$ then

$$
f\left(s_{x, y}+\gamma \cdot \partial_{x, y}+\frac{\gamma}{b-a}\right)(a, b)=0 .
$$

Indeed, similar properties occur for vector-valued polynomials: 
Lemma 7.11. Let $f \in \mathbb{C}[x, y] \otimes V_{\lambda}$ then

$$
f\left(s_{x, y} \otimes s_{x, y}+\gamma \partial_{x, y} \otimes 1+\frac{\gamma}{b-a}\right)(a, b)=0
$$

when $f(b, a)=0$.

Proof. Write $f(x, y)=\sum_{\tau} f^{\tau}(x, y) \otimes \tau$ and remark that $f(b, a)=0$ implies $f^{\tau}(a, b)=0$ for each $\tau$. But $f s_{x, y}=\sum_{\tau} f^{\tau} s_{x, y} \otimes \tau s_{x, y}$ and by Lemma $7.10 f^{\tau} s_{x, y}(a, b)=0$. Hence $f s_{x, y}(a, b)=0$. In the same way, Lemma 7.10 implies $f^{\tau}\left(\partial_{x, y}+\frac{1}{b-a}\right)(a, b)=0$ and then $f\left(\partial_{x, y} \otimes 1+\frac{1}{b-a}\right)(a, b)=0$. This proves the result.

Example 7.12. Consider the polynomial $\hat{J}_{001,{ }_{31}^{2}}$. This polynomial vanishes for $x_{3}=-2$ : $\hat{J}_{001,2}^{2}\left(x_{1}, x_{2},-2\right)=0$. Since

$$
\hat{J}_{010,2}^{2}=\hat{J}_{001,2}^{2}\left(\varsigma_{21}+\frac{1}{\alpha+1}\right)
$$

one has $\hat{J}_{010,{ }_{31}^{2}}\left(x_{1},-2, \alpha-1\right)=0$. Finally,

$$
\hat{J}_{100,2}^{2}=\hat{J}_{010,2}^{2}\left(\varsigma_{1}+\frac{1}{\alpha+2}\right)
$$

implies $\hat{J}_{100,2}^{2}(-2, \alpha, \alpha-1)=0$.

Denote by $\Phi^{+}$the operator defined by $\left[x_{1}, \ldots, x_{N}\right] \Phi^{+}=\left[x_{2}, x_{3}, \ldots, x_{N}, x_{1}+\alpha\right]$. The action of the affine operator $\hat{\Psi}$ propagates information about vanishing properties:

Lemma 7.13. One has

$$
f \hat{\Psi}\left(\left[a_{1}, \ldots, a_{N}\right] \Phi^{+}\right)=0
$$

when $f\left(a_{1}, \ldots, a_{N}\right)=0$.

Hence, for each pair $(v, \tau)$ one can compute at least one $N$-tuple $\left(a_{1}^{v, \tau}, \ldots, a_{N}^{v, \tau}\right)$ such that $J_{v, \tau}\left(a_{1}^{v, \tau}, \ldots, a_{N}^{v, \tau}\right)=0$.

Proposition 7.14. Denote by $\mathbf{V}_{v, \tau}$ the vector whose $i$-th component is

$$
\mathbf{V}_{v, \tau}[i]:=\alpha\left(v^{+}[1]-v[i]\right)+\left(\mathrm{CT}_{\tau}[1]-\mathrm{CT}_{\tau}\left[\sigma_{v}[i]\right]\right)-N+1 .
$$

Let $m$ be the smallest integer such that $v[m]=\max \{v[i]: 1 \leq i \leq N\}$. One has

$$
\hat{J}_{v, \tau}\left(x_{1}, \ldots, x_{m-1}, \mathbf{V}_{v, \tau}[m], \ldots, \mathbf{V}_{v, \tau}[N]\right)=0
$$

Proof. Denote by $\tilde{\Phi}$ the operator defined by

$$
\left[x_{1}, \ldots, x_{N}\right] \tilde{\Phi}= \begin{cases}{\left[x_{1}, \ldots, x_{N}\right] \Phi} & \text { if } x_{1}=a \alpha+b \text { with } a>0 \\ {\left[x_{2}+\alpha, \ldots, x_{N}+\alpha, x_{1}\right]} & \text { otherwise }\end{cases}
$$

Observe first that the vectors $\mathbf{V}_{v, \tau}$ are obtained by substituting $\Psi$ by $\tilde{\Phi}$ in $G_{\tau}$ :

Lemma 7.15. Recall the notation $\left[v_{1}, \ldots, v_{N}\right] \Psi=\left[v_{2}, \ldots, v_{N}, v_{1}+1\right]$. One has

$$
\mathbf{V}_{v \Psi, \tau}=\mathbf{V}_{v, \tau} \tilde{\Phi}
$$


Proof. Let $m$ be the smallest integer such that $v[m]=\max \{v[i]: 1 \leq i \leq N\}$. If $m>1$ then $v^{+}[1]=v \Psi^{+}[1]$. It follows that $\mathbf{V}_{v \Psi, \tau}[i]=\mathbf{V}_{v, \tau}[i+1]$ if $i<N$ and, since $v \Psi[N]=v[1]+1$ one has $\mathbf{V}_{v \Psi, \tau}[N]=\mathbf{V}_{v, \tau}[1]-\alpha$. That is $\mathbf{V}_{v \Psi, \tau}=\mathbf{V}_{v, \tau} \Phi$. If $m=1$ then $v^{+}[1]=(v \Psi)^{+}[1]-1$. Hence, $\mathbf{V}_{v \Psi, \tau}[i]=\mathbf{V}_{v, \tau}[i+1]+\alpha$ for $i<N$ and $\mathbf{V}_{v \Psi, \tau}[N]=\mathbf{V}_{v, \tau}[1]$. This ends the proof.

Hence, using Lemma 7.15, a straightforward induction shows that

$$
\mathbf{V}_{v, \tau}[m]=1-N
$$

Let us prove the proposition by induction on the length of the path from $\hat{J}_{0 \ldots 01, \tau}$ to $\hat{J}_{v, \tau}$. First note that

$$
\mathbf{V}_{0 \ldots 01, \tau}[N]=-N+1
$$

implies $\hat{J}_{0 \ldots 01, \tau}\left(x_{1}, \ldots, x_{N-1}, \mathbf{V}_{0 \ldots 01, \tau}[N]\right)=0$, since

$$
\hat{J}_{0 \ldots 01, \tau}=\hat{J}_{0 \ldots 0, \tau} \hat{\Psi}=\hat{J}_{0 \ldots 0, \tau} T\left(x_{N}-N+1\right) .
$$

Suppose that there is an arrow

$$
\left(\tau, \zeta^{\prime}, v^{\prime}, \sigma^{\prime}\right) \rightarrow s_{i} \longrightarrow(\tau, \zeta, v, \sigma)
$$

in $G_{\tau}$. Let $m^{\prime}$ be the smallest integer such that $v^{\prime}\left[m^{\prime}\right]=\max \left\{v^{\prime}[i]: 1 \leq i \leq N\right\}$. We have by induction,

$$
\hat{J}_{v^{\prime}, \tau}\left(x_{1}, \ldots, x_{m^{\prime}-1}, \mathbf{V}_{v^{\prime}, \tau}\left[m^{\prime}\right], \ldots, \mathbf{V}_{v^{\prime}, \tau}[N]\right)=0 .
$$

By hypothesis, $i \neq m^{\prime}$, otherwise $v^{\prime}[i] \geq v^{\prime}[i+1]$ and the arrow is not in $G_{\tau}$. Furthermore we have:

$$
\begin{aligned}
\hat{J}_{v, \tau} & =\hat{J}_{v^{\prime}, \tau}\left(s_{i} \otimes s_{i}+\partial_{i} \otimes 1+\frac{1}{\left(v^{\prime}[i+1]-v^{\prime}[i]\right) \alpha+\left(\mathrm{CT}_{\tau}\left[\sigma_{v^{\prime}}[i+1]\right]-\mathrm{CT}_{\tau}\left[\sigma_{v^{\prime}}[i]\right]\right)}\right) \\
& =\hat{J}_{v^{\prime}, \tau}\left(s_{i} \otimes s_{i}+\partial_{i} \otimes 1+\frac{1}{(v[i]-v[i+1]) \alpha+\left(\mathrm{CT}_{\tau}\left[\sigma_{v}[i]\right]-\mathrm{CT}_{\tau}\left[\sigma_{v}[i+1]\right]\right)}\right) .
\end{aligned}
$$

Consider three cases

1. If $i<m^{\prime}-1$ then Lemma 7.11 implies

$$
\hat{J}_{v, \tau}\left(x_{1}, \ldots, x_{m-1}, \mathbf{V}_{v^{\prime}, \tau}\left[m^{\prime}\right], \ldots, \mathbf{V}_{v^{\prime}, \tau}[N]\right)=0 .
$$

But $\mathbf{V}_{v^{\prime}, \tau}\left[m^{\prime}, m^{\prime}+1, \ldots, N\right]=\mathbf{V}_{v, \tau}\left[m^{\prime}, m^{\prime}+1, \ldots, N\right]$ and $m^{\prime}=m$. This proves the result.

2. If $i=m^{\prime}-1$ then $m=m^{\prime}-1$ and, as a special case of (7.2), one has

$$
\hat{J}_{v^{\prime}, \tau}\left(x_{1}, \ldots, x_{m^{\prime}-2}, \mathbf{V}_{v, \tau}[m], \mathbf{V}_{v^{\prime}, \tau}\left[m^{\prime}\right], \ldots, \mathbf{V}_{v^{\prime}, \tau}[N]\right)=0
$$

But

$$
\begin{aligned}
\mathbf{V}_{v^{\prime}, \tau}\left[m^{\prime}\right]-\mathbf{V}_{v, \tau}[m] & =\mathbf{V}_{v, \tau}[m+1]-\mathbf{V}_{v, \tau}[m] \\
& =(v[m]-v[m+1]) \alpha+\left(\mathrm{CT}_{\tau}\left[\sigma_{v}[m]\right]-\mathrm{CT}_{\tau}\left[\sigma_{v}[m+1]\right]\right) .
\end{aligned}
$$

The result is, now, a direct consequence of Lemma 7.11. 
3. If $i>m^{\prime}$ then $\mathbf{V}_{v^{\prime}, \tau}\left[m^{\prime}, m^{\prime}+1, \ldots, N\right]=\mathbf{V}_{v, \tau}\left[m^{\prime}, m^{\prime}+1, \ldots, N\right] . s_{i}$ and $m^{\prime}=m$. Remarking that,

$$
\begin{aligned}
\mathbf{V}_{v^{\prime}, \tau}[i]-\mathbf{V}_{v, \tau}[i+1] & =\mathbf{V}_{v, \tau}[i+1]-\mathbf{V}_{v, \tau}[i] \\
& =(v[i]-v[i+1]) \alpha+\left(\mathrm{CT}_{\tau}\left[\sigma_{v}[i]\right]-\mathrm{CT}_{\tau}\left[\sigma_{v}[i+1]\right]\right) .
\end{aligned}
$$

Lemma 7.11 gives the result.

Suppose that there is an arrow

$$
\left(\tau, \zeta^{\prime}, v^{\prime}, \sigma^{\prime}\right)-\Psi \longrightarrow(\tau, \zeta, v, \sigma)
$$

in $G_{\tau}$. Let $m^{\prime}$ be the smallest integer such that $v^{\prime}\left[m^{\prime}\right]=\max \left\{v^{\prime}[i]: 1 \leq i \leq N\right\}$. We have by induction,

$$
\hat{J}_{v^{\prime}, \tau}\left(x_{1}, \ldots, x_{m^{\prime}-1}, \mathbf{V}_{v^{\prime}, \tau}\left[m^{\prime}\right], \ldots, \mathbf{V}_{v^{\prime}, \tau}[N]\right)=0 .
$$

We need to consider two cases:

1. If $m^{\prime}>1$ then, since, $\hat{J}_{v, \tau}=\hat{J}_{v^{\prime}, \tau} . \hat{\Psi}$ Lemma 7.13 and equation (7.3) imply

$$
\begin{aligned}
& \hat{J}_{v, \tau}\left(x_{2}, \ldots, x_{m}, \mathbf{V}_{v, \tau}[m], \ldots, \mathbf{V}_{v, \tau}[N-1], x_{1}\right) \\
& \quad=\hat{J}_{v, \tau}\left(x_{2}, \ldots, x_{m^{\prime}-1}, \mathbf{V}_{v^{\prime}, \tau}\left[m^{\prime}\right], \ldots, \mathbf{V}_{v^{\prime}, \tau}[N], x_{1}\right)=0 .
\end{aligned}
$$

In particular,

$$
\hat{J}_{v, \tau}\left(x_{1}, \ldots, x_{m-1}, \mathbf{V}_{v, \tau}[m], \ldots, \mathbf{V}_{v, \tau}[N]\right)=0 .
$$

2. If $m^{\prime}=1$ then, from (7.1), one has $\mathbf{V}_{v^{\prime}, \tau}[1]=\mathbf{V}_{v, \tau}[N]=1-N$. But, since $\hat{J}_{v, \tau}=$ $\hat{J}_{v^{\prime}, \tau} T\left(x_{N}-N+1\right)$, one has

$$
\hat{J}_{v, \tau}\left(x_{1}, \ldots, x_{N}-1, \mathbf{V}_{v, \tau}[N]\right)=0
$$

and the result is just a special case obtained from this equality by specializing the values

\begin{tabular}{|c|c|c|c|c|}
\hline 5 & & -9 & & \\
\hline 3 & & -12 & & \\
\hline $0 \quad 5$ & and & -7 & -10 & \\
\hline 42 & $\begin{array}{ll}4 & 3\end{array}$ & -11 & -13 & $-10-8$ \\
\hline
\end{tabular}
of the $x_{i}$.

Example 7.16. One has:

$$
\mathbf{V}_{[0,2,2,1,0,3,5,1], \frac{1}{75}}=[5 \alpha-9,3 \alpha-8,3 \alpha-12,4 \alpha-10,5 \alpha-10,2 \alpha-13,-7,4 \alpha-11] .
$$

Indeed,

$$
\sigma_{[0,2,2,1,0,3,5,1]}=[7,3,4,5,8,2,1,6] \text { and }[0,2,2,1,0,3,5,1]^{+}=[5,3,2,2,1,1,0,0]
$$

and the values of $v^{+}[1]-v[i]$ and $\left(\mathrm{CT}_{\tau}[1]-\mathrm{CT}_{\tau}\left[\sigma_{v}[i]\right]\right)-N+1$ are computed by taking the corresponding values in the RST

Hence,

$$
\hat{J}_{[0,2,2,1,0,3,5,1],{\underset{75}{35}}_{8642}^{\frac{1}{3}}}\left(x_{1}, x_{2}, x_{3}, x_{4}, x_{5}, x_{6},-7,4 \alpha-11\right)=0 .
$$



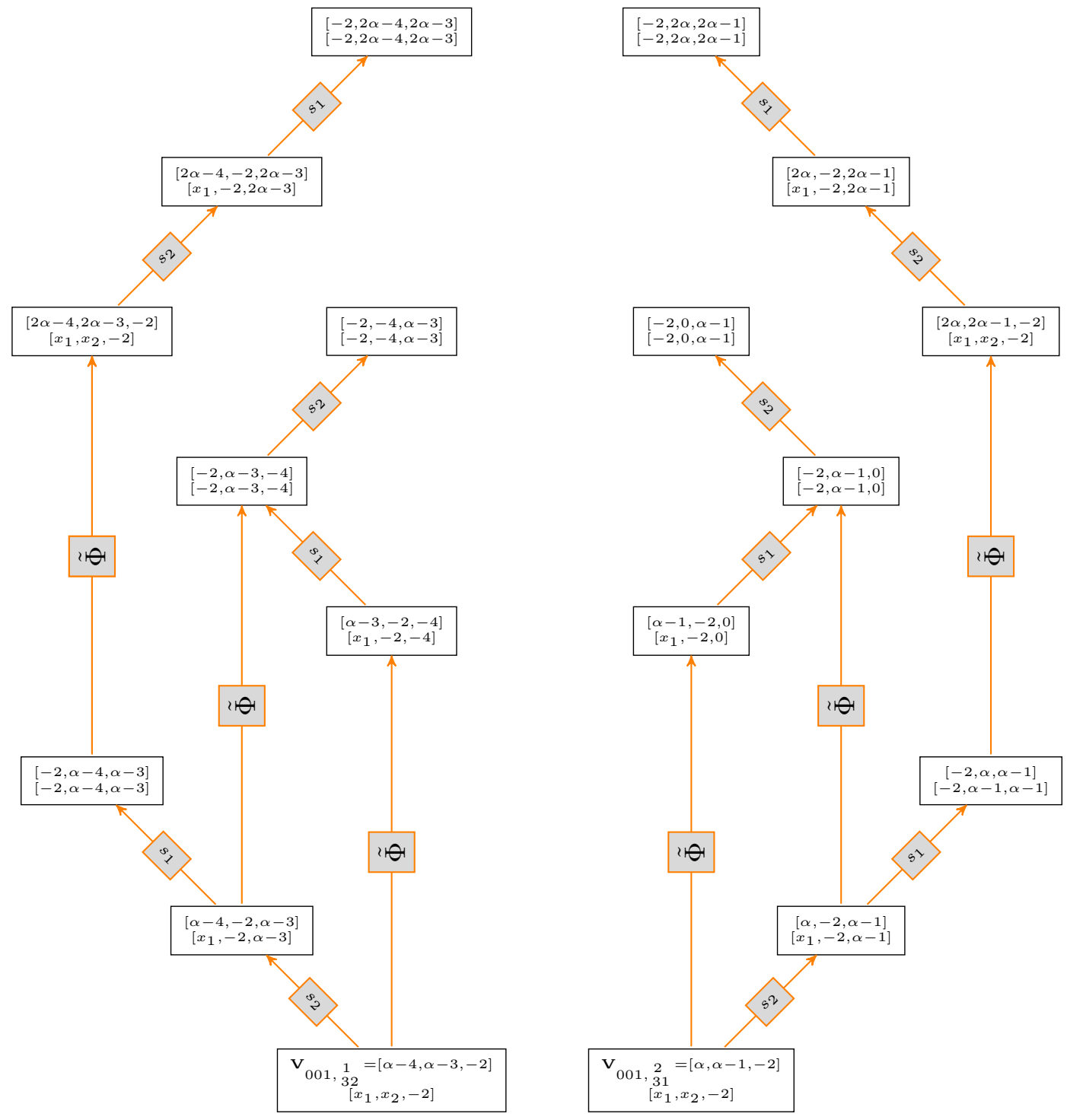

Figure 10. Propagation of some vanishing properties for $\lambda=21$.

The vanishing properties described in Proposition 7.14 are obtained by combining the actions of the $s_{i}$ and $\tilde{\Phi}$ on the initial vectors $\mathbf{V}_{0^{N}, \tau}$.

Example 7.17. Consider the propagation of vanishing properties described in Fig. 10.

Lemma 7.13 and Proposition 7.14 suggest that one can compute other vanishing properties by combining the actions of the $s_{i}$ and $\Phi^{+}$. A general closed formula remained to be found and, unfortunately, the vanishing properties obtained by propagation from $\mathbf{V}_{0^{N}, \tau}$ are not sufficient to characterize the shifted Jack polynomials.

Example 7.18. One has

$$
\hat{J}_{[002],{ }_{31}^{2}}\left(\mathbf{V}_{[001],{ }_{31}^{2}} s_{2} s_{1} \Phi^{+}\right)=\hat{J}_{[002],{ }_{31}^{2}}(\alpha, \alpha-1, \alpha-2)=0 .
$$

Note that the only vanishing property obtained by propagation for $\hat{J}_{[100],{ }_{31}^{2}}$ is

$$
\hat{J}_{[100],{ }_{31}^{2}}(-2, \alpha, \alpha-1)=0,
$$

and this is not sufficient to characterize the polynomial $\hat{J}_{[100],}{ }_{31}$. 


\section{Conclusion}

In this paper we used the Yang-Baxter graph technique to produce a structure describing the nonsymmetric Jack polynomials whose values lie in an irreducible $\mathfrak{S}_{N}$-module. The graph is directed with no loops and has exactly one root or base point. Any path joining the root to a vertex is essentially an algorithm for constructing the Jack polynomial at that vertex, and the edges making up the path are the steps of the algorithm. The edges are labeled by the generators of the braid group or by an affine operation.

These techniques are used to analyze restriction to a subgroup $\mathfrak{S}_{M}$ and also to construct symmetric and antisymmetric Jack polynomials. These are associated with certain subgraphs.

Finally the graph technique is used to construct shifted, or inhomogeneous, vector-valued Jack polynomials.

The theory is independent of the numerical value of the parameter $\alpha$ provided that the eigenspaces of $\widetilde{\xi}_{i}$ all have multiplicity one, that is, that no two vertices of the graph have the same spectral vector. Future work is needed to analyze situations where this condition is violated, in particular when $\alpha$ has a singular value, $\left\{\frac{n}{m}: 2 \leq n \leq N, m \in \mathbb{Z}, \frac{m}{n} \notin \mathbb{Z}\right\}$. There may not be a basis of Jack polynomials for the space of all polynomials. For particular choices of $\lambda$ there may exist symmetric Jack polynomials of highest weight, that is, those annihilated by $\sum_{i=1}^{N} \mathfrak{D}_{i}$. It seems plausible that any graph describing such a special case would be significantly different from $G_{\lambda}$. As a final remark, note that in the case of the trivial representation, some families of highest weight Jack polynomials have been found (see e.g. $[3,10,1])$ and related to the theory of the fractional quantum Hall effect [12].

\section{Acknowledgments}

The authors are grateful to A. Lascoux for fruitful discussions about the Yang-Baxter Graphs. The authors acknowledge the referees for their meticulous reading and valuable comments. This paper is partially supported by the ANR project PhysComb, ANR-08-BLAN-0243-04.

\section{References}

[1] Baratta W., Forrester P.J., Jack polynomials fractional quantum Hall states and their generalizations, Nuclear Phys. B 843 (2011), 362-381, arXiv:1007.2692.

[2] Baker T.H., Forrester P.J., Symmetric Jack polynomials from non-symmetric theory, Ann. Comb. 3 (1999), 159-170, q-alg/9707001.

[3] Bervenig B.A., Haldane F.D.M., Clustering properties and model wave functions for non-abelian fractional quantum Hall quasielectrons, Phys. Rev. Lett. 102 (2009), 066802, 4 pages, arXiv:0810.2366.

[4] Dunkl C.F., Symmetric and antisymmetric vector-valued Jack polynomials, Sém. Lothar. Combin. 64 (2010), Art. B64a, 31 pages, arXiv:1001.4485.

[5] Dunkl C., Griffeth S., Generalized Jack polynomials and the representation theory of rational Cherednik algebras, Selecta Math. (N.S.) 16 (2010), 791-818, arXiv:1002.4607.

[6] Etingof P., Stoica E., Unitary representation of rational Cherednik algebras, With an appendix by Stephen Griffeth, Represent. Theory 13 (2009), 349-370, arXiv:0901.4595.

[7] Griffeth S., Jack polynomials and the coinvariant ring of $G(r, p, n)$, Proc. Amer. Math. Soc. 137 (2009), 1621-1629, arXiv:0806.3292.

[8] Griffeth S., Orthogonal functions generalizing Jack polynomials, Trans. Amer. Math. Soc. 362 (2010), 6131-6157, arXiv:0707.0251.

[9] Knop F., Sahi S., A recursion and a combinatorial formula for Jack polynomials, Invent. Math. 128 (1997), 9-22, q-alg/9610016. 
[10] Jolicoeur T., Luque J.-G., Highest weight Macdonald and Jack polynomials, J. Phys. A: Math. Theor. 44 (2011), 055204, 21 pages, arXiv:1003.4858.

[11] Jucys A.-A.A., Symmetric polynomials and the center of the symmetric group ring, Rep. Math. Phys. 5 (1974), 107-112.

[12] Laughlin R.B., Anomalous quantum hall effect: an incompressible quantum fluid with fractionally charged excitations, Phys. Rev. Lett. 50 (1983), 1395-1398.

[13] Lascoux A., Young's representation of the symmetric group, available at http://www-igm.univ-mlv.fr/ al/ARTICLES/ProcCrac.ps.gz.

[14] Lascoux A., Yang-Baxter graphs, Jack and Macdonald polynomials, Ann. Comb. 5 (2001), 397-424.

[15] Macdonald I.G., Symmetric functions and Hall polynomials, 2nd ed., Oxford Mathematical Monographs, Oxford Science Publications, The Clarendon Press, Oxford University Press, New York, 1995.

[16] Macdonald I.G., Affine Hecke algebras and orthogonal polynomials, Cambridge Tracts in Mathematics, Vol. 157, Cambridge University Press, Cambridge, 2003.

[17] Murphy G.E., A new construction of Young's seminormal representation of the symmetric groups, J. Algebra 69 (1981), 287-297.

[18] Okounkov A., Vershik A., A new approach to the representation theory of the symmetric groups. II, J. Math. Sci. (N.Y.) 131 (2005), no. 2, 5471-5494, math.RT/0503040.

Okounkov A., Vershik A., A new approach to representation theory of symmetric groups, Selecta Math. (N.S.) 2 (1996), 581-605. 